\title{
Avenir de la recherche et Maisons des sciences de l'Homme
}

\author{
Sous la direction de \\ Jacques Commaille
}
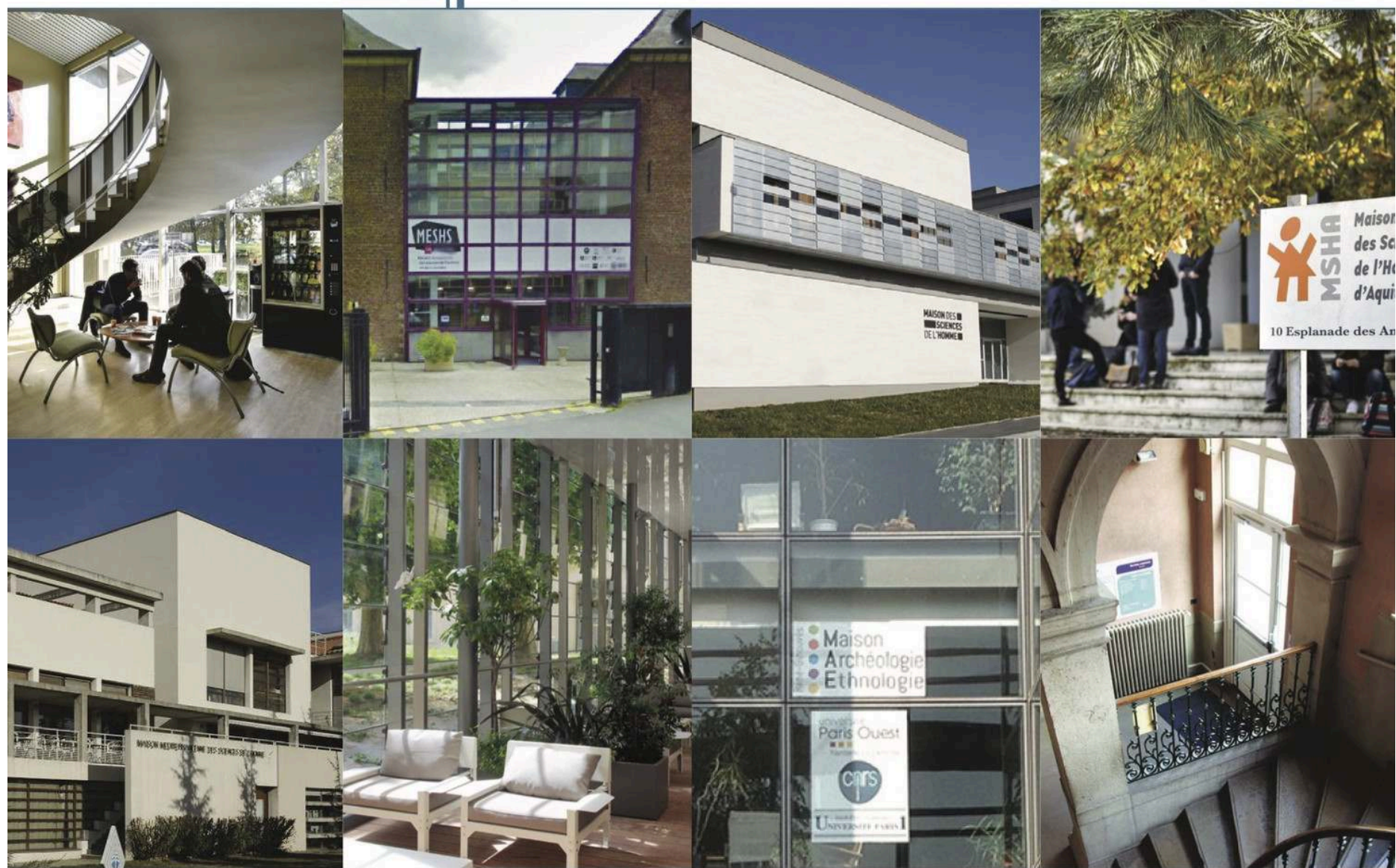

En partenariat avec $\longrightarrow$ ? Reseau $\mathrm{MSH}$ 


\title{
Avenir de la recherche et Maisons des sciences de l'Homme
}

\author{
Réédition 2020
}

Jacques Commaille (dir.)

DOI : 10.4000/books.allianceathena.352

Éditeur : Éditions A. Athéna

Lieu d'édition : Paris

Année d'édition : 2020

Date de mise en ligne : 19 janvier 2021

Collection : Collection ATHENA

EAN électronique : 9791093170060

\section{O OpenEdition}

\section{Books}

https://books.openedition.org

\section{Édition imprimée}

EAN (Édition imprimée) : 9791093170053

Nombre de pages : 142

\section{Référence électronique}

COMMAILLE, Jacques (dir.). Avenir de la recherche et Maisons des sciences de l'Homme : Réédition 2020.

Nouvelle édition [en ligne]. Paris : Alliance Athena, 2020 (généré le 19 avril 2023). Disponible sur

Internet : <http://books.openedition.org/allianceathena/352>. ISBN : 9791093170060 . DOI : https:// doi.org/10.4000/books.allianceathena.352. 


\section{RÉSUMÉS}

Cette réédition, en 2020, de l'ouvrage paru en 2006 et consacré aux Maisons des sciences de l'homme permet de mettre en lumière les fondamentaux scientifiques et institutionnels à l'origine de la création des MSH et de leur réseau, déployé au cours des années 2000 sur le territoire national. Ce livre s'adresse à tous ceux qui s'intéressent aux sciences humaines et sociales et aux évolutions désicives pour les acteurs français. Il est destiné à être complété par un deuxième volume qui couvrira la période 2012-2020. C'est une initiative du Réseau national des MSH soutenue par l'alliance Athéna. 


\section{Avenir de la recherche et Maisons des sciences de l'Homme}

Sous la direction de Jacques Commaille

Réédition 2020
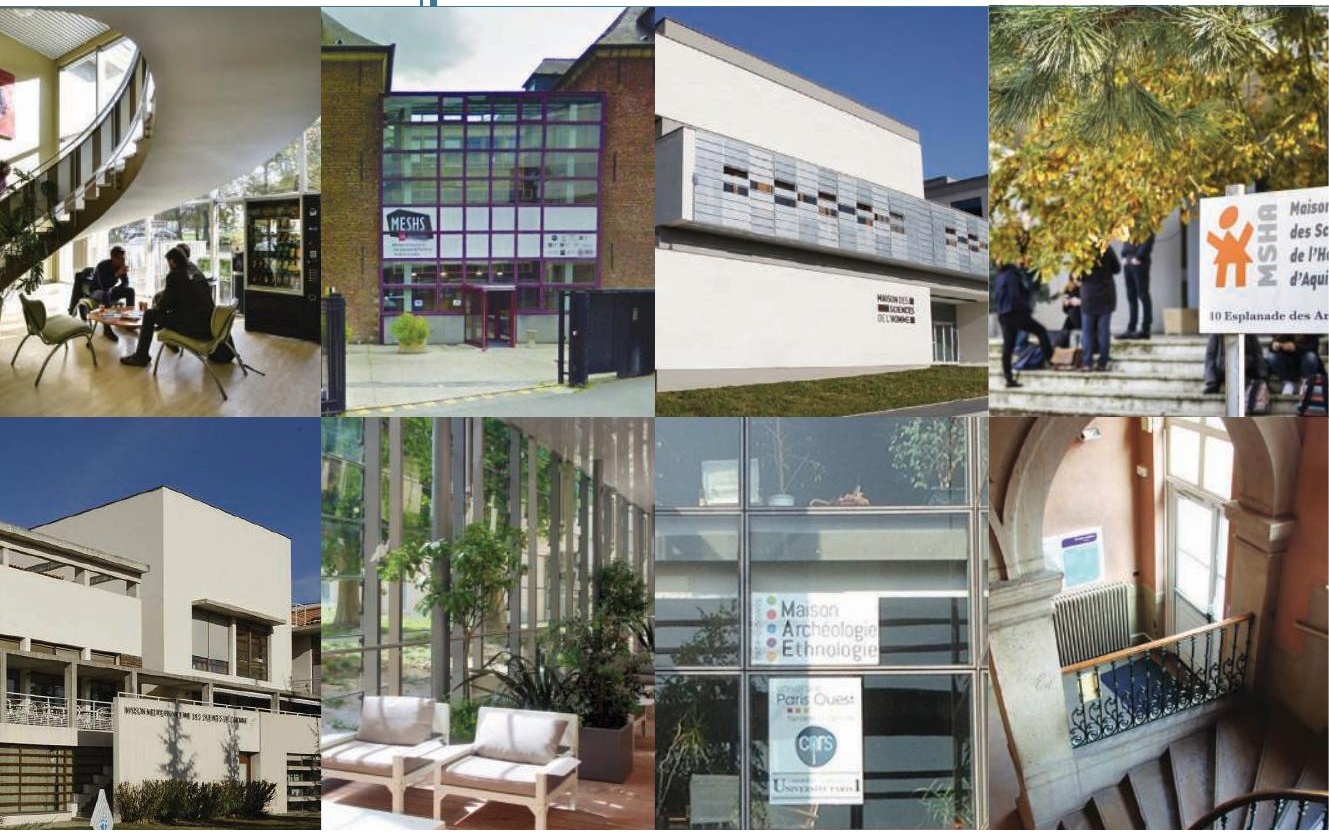

En partenariat

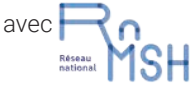




\section{Collection ATHENA}

imaginée et dirigée par

Françoise Thibault.

Disputatio autour de la politique scientifique pensée comme un art d'organiser et de développer la recherche au bénéfice de la connaissance et des citoyens.

\section{Dans la même collection}

Des sciences dans la Science, Ouvrage dirigé par Jacques Commaille et Françoise Thibault

L'énergie des sciences sociales, Ouvrage dirigé par Olivier Labussière et Alain Nadaï

Les études aréales, Ouvrage dirigé par François-Joseph Ruggiu Le doctorat en SHS, Etat de l'art et recommandations Ouvrage coordonné par Françoise Thibault

Responsable de publication

Sarah Streliski

Direction artistique et conception graphique

Laetitia Martin

(C)Alliance Athéna, 2019

www.alliance-athena.fr

Tous droits réservés pour tous pays.

ISBN : 979-10-93170-05-3 


\section{Avenir de la recherche et Maisons des sciences de l'Homme}

Sous la direction de Jacques Commaille I 



\section{Table des matières}

Introduction 7

La politique du Réseau national des

Maisons des sciences de l'Homme

Histoire d'une structuration : de la

Maison au Réseau........................................................... 23

Les paradoxes de la dynamique des Maisons ................................. 24

Les Maisons des sciences de l'Homme,

une histoire de générations.

Les Maisons de la deuxième génération

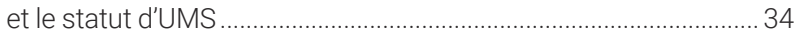

Sur le rôle du CNRS................................................................................... 36

Les Maisons de la troisième génération,

le rôle du Fonds national de la science et du COS ........................... 39

Sur le rôle des collectivités locales .......................................................... 45

Sur l'historique du Réseau ..................................................................... 46

Les années 1999-2001 de l'ACl ........................................................... 47

La charte et les statuts du Réseau ......................................................... 48

De nouvelles perspectives pour le Réseau......................................... 50

Les principes fondateurs du Réseau national

des Maisons des sciences de l'Homme .......................... 53

Les principes fondateurs dans les textes .......................................... 54

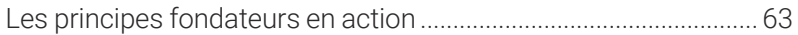

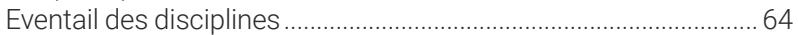

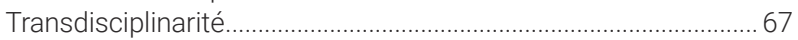

Statut des différentes disciplines......................................................6 69

Finalités et évaluation de l'interdisciplinarité .................................. 70

Dynamique interinstitutionnelle ................................................... 72

Orientation internationale ...................................................................... 77

Inscription politique et sociale/implantation locale et régionale ... 84

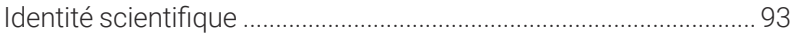

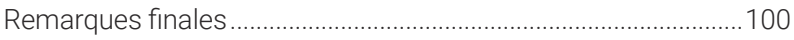

Enjeux de l'évaluation des

Maisons des sciences de l'Homme ............................... 103

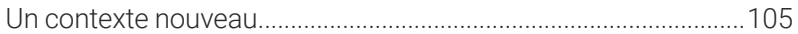

La diversité des statuts .................................................................... 106 
Instruments et procédés d'évaluation

du conseil d'orientation scientifique ............................................114

Les appels d'offres ..........................................................................118

Lévaluation comme instrument de pilotage:

bilan et perspectives

Charte du Réseau des Maisons

des sciences de l'Homme.............................................. 131

Carte du Réseau des Maisons

des sciences de l'Homme.

Liste des contributeurs ............................................... 138

Bibliographie............................................................. 140 


\section{Introduction |}

Pascal Buléon, Gilles Pollet, Nicolas Thély I

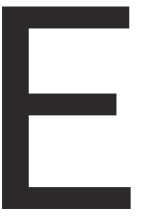

n 2006, Jacques Commaille, Pierre Rouillard et Serge Wolikow écrivaient : « La politique nationale de développement des MSH est une réalité et un projet». Aujourd'hui, comme ils le souhaitaient, ce projet est « totalement inscrit dans la réalité » nationale de la recherche en sciences humaines et sociales.

Si nous avons fait le choix, en 2020, de rééditer l'ouvrage de 2006 consacré aux Maisons des sciences de l'homme, c'est avec la double intention de témoigner de la pérennité du projet initial et de souligner ses formidables capacités d'adaptation aux cadres institutionnels actuels.

L'histoire des MSH qui est dessinée dans le livre coordonné par Jacques Commaille témoigne des formes diverses qu'ont prises les différentes Maisons sur le territoire national. Attribuant à 
Fernand Braudel la paternité de l'idée de MSH, ce livre rappelle sa volonté scientifique ambitieuse de «supprimer les droits de douane entre disciplines ».

Les textes écrits aux racines de ce mouvement buissonnant, plein de vitalité, sont à nouveau à disposition de tous. Ils ont d'ailleurs contribué à la réflexion récente sur la place et le rôle des MSH dans le paysage français de l'enseignement supérieur et de la recherche.

La réflexion collective ${ }^{2}$ conduite en 2019, avec pour objectifs l'actualisation de la charte des MSH et la réécriture de la convention du groupement d'intérêt scientifique "Réseau des MSH », a été menée dans le même esprit d'ouverture et de respect de la diversité. Elle a permis de redessiner les contours des Maisons en en faisant des acteurs majeurs de la mise en œuvre de la politique de site en SHS, du déploiement des infrastructures de recherche et de l'essor de l'interdisciplinarité entendue dans le sens de la collaboration entre les grands domaines scientifiques.

De cette réflexion est née la conviction que le Réseau des MSH était plus nécessaire que jamais. Espace d'échanges d'expériences, il est aussi lieu de construction de compétences et de réflexions stratégiques. Le souhait d'en faire un instrument au plus près de la diversité territoriale a conduit à la création d'un directoire dont nous avons accepté la responsabilité. Cette réédition dont

\footnotetext{
2 - Présidé par Jacques Commaille, initié par le CNRS avec le soutien de l'alliance Athéna, le groupe a réuni Hamida Demirdache (CNRS), Thi-Ngeune Lo (CNRS, secrétaire de séance), Jean-François Balaudé (CPU), Jacques Dubucs (MESRI), Françoise Thibault (Alliance Athéna), Jean-Pierre Gaudin (Président du CS du RnMSH), Pascal Buléon, Gilles Pollet, Isabelle Sidéra et Nicolas Thély (directeurs et directrice de MSH), Olivier Baude (directeur de la TGIR Huma-Num), Maryline Crivello (Directrice exécutive de la Fondation A-Midex), Nélia Roulot (secrétaire générale de MSH).
} 
nous avons pris l'initiative est à l'image de notre action que nous voulons cumulative, formative et inventive. 
Avenir de la recherche 


\section{La politique du Réseau national des Maisons des sciences de l'Homme}

Jacques Commaille, Pierre Rouillard, Serge Wolikow I

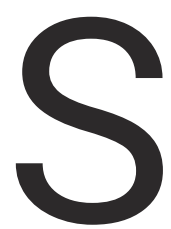

i nous devions définir d'une formule les Maisons des sciences de l'Homme (MSH) et le Réseau national qu'elles ont constitué, ainsi que la politique dont cet ensemble est issu, nous dirions que les Maisons de sciences de l'Homme assurent le regroupement, sur un même lieu, de moyens de recherche et de laboratoires comprenant des chercheurs issus de l'Université et du CNRS, appartenant à des disciplines différentes relevant des sciences de l'homme et de la société et qui sont susceptibles de s'engager conjointement sur des programmes de recherche. La création de ces Maisons des sciences de l'Homme et leur « mise en réseau » participent d'une politique de territorialisation de la recherche visant à une répartition équilibrée de pôles de recherche SHS dans l'ensemble des régions françaises avec, autant que faire se peut, une complémentarité entre ces $\mathrm{MSH}$, dans la mesure où chacune d'entre elles est susceptible de développer une dominante lui assurant 
une identité scientifique propre et, par conséquent, complémentaire aux autres.

Mais, face à ce schéma idéal, comme ne cessent de le démontrer les sciences sociales, nous savons tous que l'économie de la science est faite à la fois de poursuite de valeurs et de recherche d'intérêt. Les conditions dans lesquelles se sont créées puis développées les MSH n'échappent pas à ce constat. Si nous faisons le choix pour cette introduction de réunir nos signatures, c'est-à-dire celles de personnes qui, parmi d'autres, exercent ou ont exercé ces toutes dernières années des responsabilités dans la politique de développement des MSH au niveau national, c'est avec le désir d'affirmer fortement notre conviction quant au bien-fondé de cette politique pour l'avenir des sciences de l'homme et de la société en France, sans méconnaître ce qui demeure de faiblesses ou de lacunes liées notamment à des frilosités de territoires disciplinaires ou institutionnels, à des repliements... ou à la nécessité de faire face prioritairement à l'extrême dénuement matériel dans lequel se trouvent certaines institutions de recherche. La politique nationale de développement des MSH est une réalité et un projet. Notre souhait le plus vif est que ce qui est encore en projet s'inscrive totalement dans la réalité.

L'historique des $\mathrm{MSH}$, qui est rappelé dans l'une des contributions à la présente publication, témoigne parfaitement de la nature complexe de ce que nous pourrions appeler cette forme originale d'organisation du travail scientifique. Les raisons qui ont présidé à la création des MSH sont très différentes. Certes dans l'esprit de Fernand Braudel, le véritable créateur de l'idée de MSH, il s'agissait bien d'un grand projet intellectuel conforme à la vision magnifiquement ambitieuse inspirant son œuvre et notamment son désir de 
"supprimer les droits de douane entre disciplines». Mais les conditions dans lesquelles se sont mises en place effectivement les MSH montrent qu'à ce projet se mêlent souvent des raisons matérielles : celles par exemple d'assurer légitimement aux structures de recherche des universités des conditions décentes de travail avec, par conséquent, un impératif « d'hôtellerie » qui peut primer sur la volonté de promouvoir un projet scientifique, celles pour les tutelles locales de donner une image positive de leur action en matière de politique de recherche, sans trop se préoccuper de la mise en œuvre des principes fondateurs de la politique des MSH, celles pour les laboratoires de bénéficier d'une infrastructure de qualité et d'une mutualisation des services, sans jouer véritablement le jeu de l'inscription dans des programmes transversaux.

L'expérience que nous avons acquise dans le cadre de nos responsabilités institutionnelles nous conduit à dire que cette histoire des MSH s'inscrit dans un mouvement et que celui-ci est probablement précurseur pour l'ensemble du potentiel de recherche français en sciences de l'homme et de la société. La charte du Réseau national des MSH, qui a été signée en juin 2000 par toutes les parties prenantes et à laquelle doit adhérer chaque MSH si elle souhaite être membre du réseau, promeut des principes susceptibles d'assurer l'avènement d'un nouveau mode de production des connaissances en SHS et qui servent de référence à toute l'activité des $\mathrm{MSH}$, soit réellement, soit potentiellement. Ce qu'on a ainsi appelé la politique des trois « $i$ » en constitue une illustration éclatante.

L'interdisciplinarité apparaît comme le mariage entre des cadres conceptuels, des méthodologies permettant d'établir un pont entre les disciplines et un croisement des savoirs. Elle ouvre la possibilité d'initier un partage des connaissances entre les 
chercheurs, d'approfondir la quête de sens des phénomènes qui s'imposent à l'observation comme des totalités par rapport auxquelles la division entre disciplines peut apparaître comme inappropriée, d'ouvrir de nouvelles perspectives à la recherche grâce à la confrontation des savoirs. L'interdisciplinarité, loin de remettre en cause la pertinence et la cohérence des savoirs disciplinaires, en exalte les vertus et oblige à soumettre à la délibération des divisions du travail de connaissance qui ne résultent plus parfois que de la défense de territoires institutionnels ou n'obéissent qu'à des logiques corporatistes. La vision manichéenne qui opposerait la recherche disciplinaire et la recherche interdisciplinaire apparaît finalement erronée.

La structuration de la recherche française a été fondée sur le principe de la discipline et de la spécialisation. Mais la diversification des champs de connaissance n'a pas toujours trouvé dans le système classique d'organisation de la recherche en sciences sociales et dans les humanités une solution institutionnelle adéquate à des recherches qui peuvent être à l'intersection de plusieurs disciplines ou bien naître de questions nouvelles qui interpellent de façon transversale les savoirs existants. Les MSH constituent une tentative de réponse à une évolution qui fait surgir de nouveaux besoins de connaissance. Elles sont, ou elles peuvent devenir, des creusets de la recherche interdisciplinaire dans la mesure où la possibilité leur est donnée de regrouper dans une même institution et souvent dans un même site des équipes de recherche et des chercheurs de disciplines différentes, de mettre en œuvre des formes extrêmement variées de croisement et de confrontation de points de vue scientifiques d'horizons différents, notamment dans le cadre de la réalisation de programmes de recherche. Les MSH sont ainsi en mesure de définir des problèmes et des thématiques 
qui ne peuvent être appréhendés que par plusieurs disciplines et qui, par conséquent, sont susceptibles de devenir des problèmes et des thèmes proprement SHS, c'est-à-dire humains, sociaux et culturels avant d'être historiques, juridiques ou... sémiotiques. Elles sont en mesure d'étudier et, le cas échéant, de modéliser les effets de ces pratiques interdisciplinaires de manière à comprendre quelles sont les propriétés communes, comment la collaboration peut s'agencer, quelle est la forme épistémologique et méthodologique de cette collaboration et quelles formes d'objectivité scientifique les différentes disciplines peuvent partager.

L'international est une autre dimension importante de la politique des MSH. II va de soi que les MSH n'ont pas le monopole des collaborations internationales dans le champ des SHS. Néanmoins, et de par leurs thématiques scientifiques propres, les MSH sont en mesure de construire une tradition d'ouverture sur le monde. Cette ouverture ne doit pas seulement se mesurer en termes d'outil et d'organisation mais doit être aussi au cœur de réflexions et de programmes interculturels. Présente dans la conception initiale des $\mathrm{MSH}$, cette dimension internationale se manifeste dans des programmes de recherche ou des flux d'échanges entre chercheurs qui ont été particulièrement favorisés ces dernières années grâce aux programmes Marie Curie. II est évident que, de ce point de vue, l'existence du Réseau national des MSH constitue un atout décisif et un mode d'organisation conforme aux perspectives ouvertes par le sommet de Lisbonne et la décision de mettre en place un Espace européen de la recherche (EER).

Le Réseau national des MSH permet en effet tout à la fois : une très forte implantation régionale maîtrisée et diversifiée, conforme à la volonté de faire des régions dans le cadre européen des pôles 
d'initiative ; l'exercice d'un rôle moteur au niveau national ; l'affirmation d'une position d'interlocuteur et de partenaire au niveau européen, plus largement au niveau international. La politique des MSH est ainsi en mesure d'assumer ce qui peut sembler un paradoxe: celui de rendre compatible une forte implantation locale avec une ouverture internationale, dans le cadre d'une politique coordonnée au niveau national. En évitant le risque du localisme, les MSH s'inscrivent positivement dans une évolution qui tend à faire des régions des actrices importantes de la politique de recherche, ceci moyennant un déplacement d'une conception jacobine de la recherche vers une vision davantage articulée sur des problématiques ou des sensibilités régionales en même temps qu'ouvertes sur le niveau européen et international.

L'interinstitutionnel constitue un élément stratégique de la politique des MSH. En effet, celles-ci sont fondées sur des collaborations instituées entre plusieurs universités, le CNRS, avec le soutien de collectivités locales. La politique de développement des MSH a constamment pour objectif d'engager conjointement les différentes universités présentes au niveau régional, au-delà de leurs spécialisations et éventuellement de traditions scientifiques ou culturelles qui les tenaient éloignées les unes des autres. À ce premier dépassement de clivages institutionnels s'ajoute la volonté d'assurer la meilleure coordination possible entre les universités et les structures de recherche relevant du CNRS, ce qui implique, outre la définition en commun des grandes orientations scientifiques, l'incitation au renforcement des collaborations entre enseignants-chercheurs et chercheurs dépendant du CNRS ainsi qu'une politique d'encadrement doctoral garantissant aux doctorants l'accès à des conditions de travail équivalentes à celles dont ils sont susceptibles de bénéficier dans les laboratoires de «sciences dures». De façon plus 
générale, il en résulte une position qu'on pourrait qualifier de préprofessionnalisation, susceptible de préparer le potentiel humain nécessaire à l'avenir de la recherche en sciences humaines. Une telle structuration favorise l'implication des collectivités locales qui peuvent voir dans les MSH un élément de promotion des actions politiques menées en faveur de la science au niveau régional. Ces formes d'engagement au niveau local n'excluent pas des politiques de partenariat avec des institutions extérieures (autres universités et centres de recherche français et étrangers, autres grands établissements publics de recherche, différents ministères...)

Enfin, une forte structuration au niveau local apparaît compatible avec des collaborations scientifiques nouées avec des institutions homologues, en l'occurrence avec d'autres MSH, ceci sous l'égide du Réseau national des MSH dont elles sont membres, dans le cadre de programmes de recherche au sein desquels elles sont conjointement partenaires, ou d'une politique de mutualisation des services et des équipements.

Toutes les conditions sont ainsi réunies pour que les MSH constituent un véritable instrument de dynamisation et de structuration de la recherche en sciences humaines. Rien ne l'illustre mieux que cette fonction d'incubateur qu'elles sont en mesure d'assumer. Bénéficiant d'équipements lourds en termes de bibliothèques, de documentation (accès à des bases de données documentaires, utilisation d'équipements informatiques que ce soit pour l'écrit ou l'image, avec des instruments d'analyse d'images ou de datations, production d'outils collectifs de travail performants, notamment des bases de données), mobilisant un potentiel humain de recherche aux compétences variées et complémentaires, exceptionnellement insérées dans la communauté 
de recherche nationale et internationale, les MSH sont effectivement en mesure de contribuer à l'ouverture de voies nouvelles ou de champs nouveaux de recherche portés par de nouvelles configurations de compétences incluant éventuellement de jeunes collègues ou à l'initiative de ces derniers.

Enfin, dans un contexte où l'exigence de justification de l'utilité sociale des sciences humaines se manifeste particulièrement, les $\mathrm{MSH}$, fortement implantées localement, sorte de signal scientifique au niveau régional, sont en mesure d'avoir un rayonnement au niveau de leur environnement social, économique et culturel, notamment par l'organisation d'expositions, de conférences, de colloques, de séminaires ou de rencontres avec les acteurs de la société civile, illustrant ainsi que la fonction scientifique n'est pas incompatible avec une fonction civique... surtout lorsqu'il s'agit de sciences de l'homme et de la société !

Dans le contexte actuel, la mobilisation nationale en faveur de la recherche a mis en évidence, outre la nécessité de moyens accrus pour l'activité scientifique, l'importance d'une structuration de l'effort de recherche, notamment en SHS, ce qui renforce l'actualité du Réseau des MSH. Celui-ci, après avoir connu une extension notable ces dernières années, entre aujourd'hui dans une phase de maturité lui permettant d'articuler les thématiques entre les Maisons, acquérant ainsi une force d'intervention et de proposition qui met les SHS en capacité d'affronter les appels d'offres et les programmes scientifiques au niveau européen. Le Réseau des MSH, en apportant de manière effective son soutien à la politique de coopération internationale en SHS, possède la dimension et les ressources scientifiques permettant de développer une activité internationale différenciée et pérenne qui 
prend en compte la diversité du développement des recherches en SHS aussi bien dans les pays du Nord que du Sud.

Telle est dans ses grandes lignes ce que nous pourrions nommer la philosophie générale d'une politique de développement des $\mathrm{MSH}$, dans une optique triple où, tout à la fois : nous prenons acte de ce qu'elle a déjà produit, nous tentons de la promouvoir et nous appelons de nos vœux que soient réunies les conditions de sa réussite. Pour ce qui nous concerne, les instances dont nous avons ou avons eu la responsabilité témoignent que cette politique est faite à la fois d'incitation et de concertation pour les partenaires qu'elle implique. C'est tout le sens des missions respectives du conseil des directeurs de MSH et du conseil d'orientation du Réseau national des MSH qui deviendra bientôt le conseil scientifique du Réseau national des MSH. L'objectif passionnément poursuivi est d'assurer le respect de cette philosophie générale évoquée supra et d'assumer une sorte de fonction de socialisation, de telle sorte que les principes fondateurs des MSH suscitent l'adhésion de ceux qui en sont acteurs ou de ceux qui sont susceptibles de le devenir.

C'est dans cet esprit qu'a été conçue la présente publication. Elle comporte d'abord un historique, lequel est constitutif d'une mémoire de la politique des MSH et de leur réseau national. Cet historique permet de souligner qu'au-delà des conditions diverses de leur création portée par des contextes, des acteurs, des circonstances et des volontés parfois différentes, les MSH ont vocation à se projeter dans un avenir scientifique commun. L'ouvrage comprend à la suite deux contributions rédigées par des membres étrangers - ou exerçant à l'étranger - du conseil d'orientation du Réseau national des MSH, persuadés que nous sommes que la distance est susceptible 
d'assurer la plus grande neutralité de regard possible, sur deux aspects essentiels pour l'avenir des MSH et de leur Réseau national : leurs grandes orientations scientifiques et la question de l'évaluation de l'activité de recherche en SHS. Dans sa contribution, Pierre Guibentif fait d'abord un rappel des grands principes d'origine - des «référentiels», diraient les spécialistes - des politiques publiques, représentés par les trois « $\mathrm{i}$ » évoqués supra et auxquels se sont ajoutés, en s'affirmant progressivement, deux « i » supplémentaires: l'identité scientifique de chacune des Maisons et leur insertion dans leur environnement culturel, social et politique. II se penche ensuite sur les formes de leur mise en valeur effective par les Maisons, soulignant ainsi de façon concrète les vertus de l'interdisciplinarité, les mérites mais aussi la complexité d'une gestion de l'interinstitutionnalité, le développement d'une politique d'internationalisation exemplaire, avec cette originalité que constitue une réciprocité positive entre l'ouverture internationale et l'ancrage local. Ainsi se trouve justifié le souci de l'implantation locale, le souci réel de l'affirmation d'une identité scientifique qui porte en germe une politique nationale de territorialisation des compétences scientifiques et de leur complémentarité.

Pour leur part, Birgit Arve-Parès et Linda Hantrais mettent particulièrement en valeur, dans le chapitre qu'elles ont rédigé, l'importance stratégique de l'évaluation, celle-ci étant posée dans toute sa force et sa complexité par les MSH et leur réseau national, compte tenu de l'ambition qui les inspire et de leur place au sein de configurations institutionnelles et scientifiques complexes. Tout ceci conduit en fait à poser, d'une façon qui nous paraît exemplaire, l'ensemble de la question de l'évaluation de la recherche française en sciences de l'homme et de la société. C'est notamment ce que permettent le bilan qui est ici dressé de l'expérience du conseil d'orientation 
scientifique en la matière et les suggestions dont ce bilan autorise la formulation, ceci pour aller plus loin, avec un esprit qui est moins celui du contrôle et du jugement que celui du suivi et de l'accompagnement.

En mettant en lumière cette expérience de quelques quatre années de fonctionnement du conseil d'orientation scientifique, riche de l'engagement de compétences diverses et de celui de collègues étrangers éminents, nous espérons contribuer au renforcement d'une identité forte des Maisons et de leur réseau et, au-delà, faire partager notre profonde conviction que l'enjeu est bien celui de l'avènement d'une nouvelle culture scientifique en sciences de l'homme et de la société. Notre profonde conviction que toutes les conditions doivent être réunies, au niveau des acteurs et des institutions concernés comme à celui des tutelles de la recherche française, pour que, comme nous le disions au départ de ce propos, ce qui est encore en projet dans les MSH devienne tout à fait réalité, une réalité susceptible de surcroît de rejaillir sur l'ensemble de la recherche en sciences de l'homme et de la société. 
Avenir de la recherche 


\section{2 Histoire d'une structuration
de la recherche : de la Maison
au Réseau}

Martine Bentaboulet I

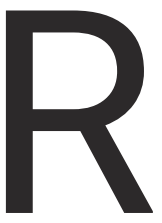

etracer I'historique des Maisons des sciences de l'Homme depuis 1963, date de la création de la première d'entre elles, la Maison des sciences de l'Homme de Paris, permet de resituer dans leur contexte les créations qui se sont succédé depuis presque un demi-siècle. S'inscrivent ainsi sur une longue période toute une série de centres de recherche en sciences humaines et sociales qui se revendiquent comme Maisons des sciences de l'Homme dans un même souci d'interdisciplinarité, d'ouverture internationale et de coopération institutionnelle. Toutes partagent l'ambition d'afficher les sciences humaines et sociales comme sciences et fondent leur identité sur un projet scientifique. II s'agit d'une aventure originale, menée souvent avec passion, toujours avec ténacité, par des personnalités scientifiques et des administrations publiques. Mettre en évidence les synergies entre les acteurs aide à comprendre comment cette politique publique de la recherche 
a été définie et mise en œuvre. L'analyse révèle que la dynamique de cette structuration est marquée par plusieurs paradoxes. Dans cette présentation, nous passerons en revue les plus marquants puis nous évoquerons quelques exemples de Maisons ${ }^{2}$ parmi les plus représentatifs, avant de décrire les événements qui ont façonné le Réseau, structure collective au moins aussi originale que les Maisons elles-mêmes et qui réunit les vingt Maisons existant à ce jour.

\section{Les paradoxes de la dynamique des Maisons}

Tout d'abord la continuité du processus est assez remarquable, le mouvement de structuration ayant bénéficié d'un appui pratiquement sans faille au niveau national de la part du CNRS et des ministères successifs en charge de la recherche et ce, pendant une période longue de cinquante ans, malgré les nombreux changements politiques intervenus et les autres types de structuration privilégiés par ailleurs. II a bénéficié aussi d'un appui très fort des nouveaux acteurs constitués par les collectivités locales à partir des années quatre-vingt-dix. Celles-ci, en effet, se sont intéressées à la construction de grands centres de recherche, dès que la recherche a été prise en compte et inscrite dans les objectifs des contrats de plan pluriannuels liant l'État et les régions (contrats de plan ÉtatRégions ou CPER). Même si l'intérêt des collectivités s'est mobilisé dans la première vague de contrats essentiellement sur des projets de sciences « dures », les vagues successives de 1989-1993 et surtout 1994-1998 permettront d'y inscrire d'ambitieux projets de Maisons des sciences de l'Homme et de rassembler les

\footnotetext{
2 - Le grand nombre de Maisons ne nous a pas permis de retracer l'histoire de chacune d'elles et les exemples choisis permettent seulement d'illustrer les grandes lignes du processus de structuration de la recherche étudié. Pour plus d'informations sur les Maisons, on pourra consulter utilement le site du Réseau www.msh-reseau.prd.fr et les lettres du département SHS du CNRS n 45 janvier 1997; n 53 février 1999 et n 68 août 2003.
} 
financements nécessaires à leur construction. Enfin, l'intérêt des universités pour les Maisons confortera leur dynamique même si, du fait de leur autonomie organisationnelle, elles ont parfois été vues par les universités comme étant sources de difficultés (en compétition par rapport aux politiques de recherche et des programmes scientifiques des universités elles-mêmes) plus que d'avantages (appui aux écoles doctorales, ouverture sur le monde socio-économique et attractivité régionale).

Un autre paradoxe réside dans le fait que, si les Maisons s'accordent aujourd'hui sur les grands principes fondateurs rappelés supra et analysés par Pierre Guibentif dans un autre chapitre (interdisciplinarité, ouverture internationale et ouverture institutionnelle, sans oublier identité scientifique et insertion régionale), il n'en reste pas moins que cette unité revendiquée s'oppose à une multiplicité de structures existantes, provenant chacune d'une histoire singulière. Si l'on regarde en effet ce qu'étaient les Maisons des sciences de l'Homme au début des années 2000, on est frappé par leur grande diversité. Certaines ont été conçues dès l'origine selon le modèle de Fernand Braudel qui inventa en 1960 le concept de Maison des sciences de l'Homme, mais beaucoup des Maisons, qui aujourd'hui revendiquent cette appellation, avaient à l'origine des objectifs très éloignés des principes «fondateurs » qui caractérisent les Maisons. Ceux-ci n'ont d'ailleurs été institutionnalisés qu'au début des années 2000, dans une charte dite «charte des Maisons des sciences de l'Homme » annexée à la convention du réseau qui lie entre elles les Maisons - ou leurs institutions de tutelle dans le cas des Maisons qui n'ont pas d'autonomie juridique. Ainsi, parmi les Maisons actuelles, certaines ont été qualifiées de " généralistes » parce que regroupant des équipes de différentes disciplines. D'autres sont à l'origine des centres de recherche thématiques (la Maison 
méditerranéenne des sciences de l'Homme à Aix, la Maison Orient Méditerranée à Lyon ou la Maison des sciences de la ville à Tours), d'autres à l'origine des centres de recherche disciplinaires (la Maison de l'archéologie et de l'ethnologie à Nanterre). D'autres encore étaient conçues comme de simples lieux d'hébergement d'équipes de recherche et de services universitaires pour, dans un souci d'« hôtellerie», répondre aux carences de locaux particulièrement criantes dans certaines universités de sciences humaines (la Maison de la recherche de Toulouse le Mirail). Sur le plan administratif, les statuts des Maisons ne sont pas non plus uniformes et leur nature tient le plus souvent au contexte historique de la création de la Maison : fondation, association loi de 1901, groupement d'intérêt public, fédération de recherche, unité mixte de service le plus souvent, ou groupement d'intérêt scientifique.

Un autre paradoxe du mouvement qui a vu se constituer cet ensemble de Maisons est qu'il n'est absolument pas uniforme dans le temps. En effet, sur une première période de trente ans, seules six Maisons vont être créées ${ }^{3}$ : à Paris en 1963, à Bordeaux et Strasbourg en 1978, à Lyon en 1975 (la Maison de l'Orient et de la Méditerranée ou MOM) et en 1989 (la Maison Rhône-Alpes des sciences de l'Homme ou MRASH), enfin à Nantes en 1993 (la Maison Ange-Guépin). Ce sont les Maisons que l'on pourrait appeler, de la « première génération ». Cette période laissera place à deux phases d'accélération très importante sous l'impulsion des tutelles de la recherche, le CNRS d'abord, le ministère de la Recherche ensuite. Elles vont se succéder et voir la création de quinze nouvelles Maisons en quinze ans.

3 - Les dates de création retenues sont celles des structures administratives correspondantes. Celles-ci interviennent bien sûr plusieurs années après que les projets de Maison aient vu le jour. 
Dans la première phase de cette accélération, on retrouve un certain nombre d'universités qui souhaitent développer de nouvelles surfaces de recherche ou « Maisons de la recherche » pour rassembler des équipes dispersées et sans moyens de travail suffisants. Dans le même temps, le CNRS est désireux, lui, de mener une politique de développement scientifique en régions hors lle-deFrancet. II va s'appuyer pour cela sur les projets des universités les plus volontaristes et sur le ministère en charge de la recherche. Cette première phase, de 1993 à 1998, concerne les projets de Caen, Poitiers, Aix et Toulouse. Pendant cette période, la MRASH se scinde en deux Maisons distinctes, l'une à Lyon, l'autre à Grenoble. La Maison archéologie ethnologie à Nanterre appartient aussi à cette période, bien qu'il s'agisse d'une Maison située en Ile-de-France. Elle représente en fait un choix politique majeur du département SHS du CNRS, qui voit là l'occasion de conclure la structuration d'une discipline particulière, l'archéologie, en grands centres de recherche déjà organisés comme tels en province. Durant cette période, une Maison des sciences de la ville voit aussi le jour à Tours.

La seconde phase d'accélération se situe entre 1998 et 2005. Elle se distingue de la précédente, car c'est sous l'impulsion principale du ministère en charge de la recherche et non plus du CNRS que vont se structurer huit autres Maisons, à Strasbourg (en remplacement

\footnotetext{
4 - II s'agit de conforter la recherche hors Ile-de-France à la fois pour développer l'efficacité de la recherche grâce à un plus grand nombre de pôles de recherche de visibilité internationale et pour accroître les partenariats avec les collectivités locales de plus en plus désireuses d'accueillir des centres de recherche de haut niveau. Le CNRS s'est ainsi engagé dans une politique de localisation en régions de chercheurs, d'équipes et de centres de recherche, associée à des transferts d'emplois dans le cadre de la politique de délocalisation imposée par le gouvernement d'Edith Cresson à tous les ministères. Pour le CNRS, les Maisons des sciences de l'Homme de cette génération représentent la contribution du département scientifique SHS, dirigé alors par A. d'Iribarne, à cette politique.
} 
de la Maison initiale qui n'a pas réussi à s'imposer sur la scène de la recherche), Paris-Nord (Saint-Denis), Nice, Lille, Dijon, Montpellier, Besançon et Clermont-Ferrand, mouvement qui se poursuit aujourd'hui encore avec un projet de Maison à Rennes et un autre à Nancy. Fin 1998, le ministre de la Recherche, Claude Allègre, multiplie les preuves de son soutien aux Maisons des sciences de l'Homme en exhortant les grandes métropoles universitaires où il n'existe pas encore de MSH à concevoir des projets de ce type.

Pour la politique affichée par le ministère, le plan Université du troisième millénaire (U3000) et les contrats de plan État-Régions en cours de négociation constituent un cadre pluriannuel privilégié. Seront ainsi inscrits dans les CPER toute une série de projets de Maisons, leur réalisation effective étant conditionnée à une évaluation scientifique ultérieure ${ }^{5}$. En complément des moyens destinés aux constructions et aux équipements, le ministère crée le Fonds national de la science (FNS), destiné à financer une politique de programmes scientifiques prioritaires. Parmi ces programmes, appelés aussi Actions concertées incitatives ( $\mathrm{ACl}$ ), un programme est spécifiquement créé pour développer un réseau entre les Maisons des sciences de l'Homme, conçu par le ministre de l'époque comme une sorte de «laboratoire sans murs ». Le CNRS n'est pas au cœur de la politique de structuration des nouvelles Maisons comme il a pu l'être précédemment mais il l'accompagne et la soutient, lui conférant par là même une crédibilité scientifique et des moyens. La question, cruciale, de l'évaluation (voir le chapitre rédigé par Linda Hantrais et Birgit Arve-Parès et la note en bas de page) liée à l'autonomisation du

5 - Ces évaluations seront conduites dans un premier temps par le conseil scientifique de l'ACI Réseau des MSH, puis à partir de 2001 par le conseil d'orientation du réseau (COS), instances mises en place par le ministère de la Recherche et conférant au Réseau une relative autonomie. 
Réseau explique que le CNRS souhaite conserver son libre choix en affichant seulement une politique de soutien.

\section{Les Maisons des sciences de l'Homme, une histoire de générations}

\section{Les Maisons de la première génération}

À tout seigneur tout honneur, la première Maison est la Maison des sciences de l'Homme de Paris, créée sous forme d'une fondation reconnue d'intérêt public. Dans un article paru dans la lettre du département SHS en août 2003', Maurice Garden la décrit ainsi : "Dans la décennie 1960 l'historien Fernand Braudel qui venait de créer l'École des hautes études en sciences sociales, par transformation en grand établissement autonome de l'ancienne sixième section de l'École pratique des hautes études, invente le concept de Maison des sciences de l'homme, formule totalement neuve et originale en France d'une institution scientifique adossée à une fondation reconnue d'utilité publique ». La fondation voit le jour grâce à des capitaux privés... américains et la Maison des sciences de l'Homme s'installe rapidement dans un immeuble construit pour elle boulevard Raspail.

Le concept de Fernand Braudel est remarquable parce que complètement novateur pour l'époque. D'une part, il conçoit une structure collective de travail au service des chercheurs (bibliothèque, lieux de rencontre, moyens financiers pour des échanges internationaux...), alors que dans beaucoup de disciplines des sciences de l'Homme, les chercheurs sont le plus souvent défenseurs d'un travail purement individuel. D'autre part, Fernand Braudel, qui est 
un farouche partisan du décloisonnement des disciplines, s'inscrit en opposant radical de la toute puissante Sorbonne.

Il lui faudra compter avec les grandes institutions en SHS contemporaines de la Maison des sciences de l'Homme de Paris, qui sont d'une part l'École des hautes études en sciences sociales, créée comme grand établissement d'enseignement et de recherche, un statut qui lui accorde la personnalité morale, d'autre part l'Institut d'études politiques "Sciences Po», placé sous la tutelle de la Fondation nationale des sciences politiques. Ces trois grands établissements, logés dans un périmètre très proche, entre le boulevard Raspail et le boulevard Saint-Germain constituent, avec l'École normale supérieure «UIm », un pôle d'attractivité exceptionnel pour la recherche en SHS, dont le poids sera très difficile à contrebalancer au fil des décennies ultérieures.

Cependant, le ministère de la Recherche de l'époque, déjà soucieux de ne pas être accusé de privilégier uniquement la création de grands centres parisiens et peut-être aussi désireux précisément de rééquilibrer le potentiel au profit des régions, ne donne son accord au projet de Fernand Braudel qu'en échange d'un engagement de sa part à promouvoir l'implantation de centres similaires à celui de la Maison de Paris en province. Fernand Braudel convaincra sans trop de difficultés des collègues universitaires de Bordeaux et Strasbourg de tenter l'aventure et dès lors l'idée d'un réseau entre la Maison mère, celle de Paris, et les nouvelles Maisons est née?. Les Maisons de Bordeaux et

\footnotetext{
7 - Pour preuve de ces liens d'origine, la présence d'un représentant des Maisons de Bordeaux et de Strasbourg dans le conseil d'administration de la Fondation de Paris. Ultérieurement, les Maisons juridiquement autonomes que sont la Maison Ange-Guépin à Nantes et la MRASH à Lyon seront aussi représentées dans ce même conseil.
} 
Strasbourg ont été dotées du statut d'association loi de 1901. N'ayant pas les moyens légaux et financiers de recruter leur personnel administratif par elles-mêmes, elles s'appuieront pour cela sur la Maison de Paris. Si l'implication du CNRS dans les Maisons de Paris, Bordeaux et Strasbourg ${ }^{8}$ 'a pas eu la même ampleur que pour d'autres, le CNRS est néanmoins membre du conseil d'administration de la Maison de Paris et représenté dans celui de la Maison de Bordeaux. Pour ce qui concerne la MSH Paris, des relations existent également avec le CNRS dans le cadre d'opérations ponctuelles donnant lieu à des conventions, par exemple pour l'accueil de chercheurs étrangers (bourses Diderot) ou l'hébergement d'unités de recherche reconnues par le CNRS.

La Maison de l'Orient et de la Méditerranée à Lyon a été créée en 1975 sous la forme d'une fédération de recherche (FR). Comme toutes les structures de ce type, elle réunit en un même lieu plusieurs unités de recherche qui conservent leurs liens avec le département du CNRS et leur université de rattachement. L'activité de recherche des unités composantes est évaluée séparément de celle de la structure fédérative. La Fédération de recherche est une structure mixte CNRS-université qui, comme les unités de recherche, ne possède pas la personnalité morale. La MOM a été conçue par Jean Pouilloux comme une Maison spécialisée et un pôle de compétences des sciences de l'Antiquité du bassin oriental de la Méditerranée, avec des équipes couvrant un champ à la fois géographique et chronologique large. Elle n'est pas à l'origine

8- Nous faisons référence ici à la première expérience de Strasbourg. Celle-ci a depuis pris une forme différente dans le cadre de ce qui est devenu la Maison interuniversitaire des sciences de l'Homme d'Alsace. 
une Maison des sciences de l'Homme mais a souhaité être reconnue comme telle?

Deux Maisons des sciences de l'Homme seront créées plus tard, en 1980 et 1993, et seront dotées d'un statut leur accordant la personnalité morale, sous la forme d'un groupement d'intérêt public ou GIP. Ce sont la Maison Rhône-Alpes des sciences de l'Homme à Lyon, en 1989, et la Maison Ange-Guépin à Nantes, en 1993. Plusieurs facteurs ont été déterminants dans le projet de la MRASH proposé à l'époque par Maurice Garden, professeur des universités, historien et vice-président de l'université de Lyon II. Les motivations de ce projet, qui est avant tout une initiative locale, sont d'abord les conditions de travail déplorables des chercheurs de l'université de Lyon en SHS : «On vivait dans des conditions de placard, soit dans des sous-sols soit dans les combles des bâtiments de façon absolument scandaleuse ${ }^{10}$ ». L'opportunité de récupérer pour la recherche des locaux de la ville, libérés dans le centre de Lyon par suite du déménagement des services qui les occupaient, est d'emblée saisie par Maurice Garden lorsqu'elle se présente.

La création à Lyon, quelques années auparavant, d'une Maison thématique par Jean Pouilloux, la Maison Orient Méditerranée, incite M. Garden à proposer à la municipalité un projet « en jouant la carte de la recherche collective, avec une douzaine d'équipes CNRS en dehors de la Maison de l'Orient et en mettant en avant un besoin de locaux pour les économistes, les littéraires, les linguistes, les psychologues, les historiens, les géographes. Nous avons dit que nous

\footnotetext{
9 - La MOM participera par exemple à la réunion organisée à Paris en 1999 sous l'impulsion de la direction de la recherche au ministère de la Recherche, dans le but d'harmoniser les objectifs généraux des MSH et de préparer leur mise en réseau. Cette réunion faisait suite à une rencontre organisée par les directeurs eux-mêmes des « Maisons de la recherche en SHS » à Poitiers en 1998.
}

10 - Entretien avec Maurice Garden, octobre 2001. 
étions d'accord pour qu'il n'y ait pas seulement Lyon II, mais Lyon II et Lyon III. On ne se parlait pas à l'époque mais on commençait à dire que c'était peut-être possible. Cela a été soumis aux instances des universités... II y avait $3000 \mathrm{~m}^{2}$. II était prévu d'en faire une Maison des sciences de l'Homme. C'est le terme qui avait été choisi. ${ }^{11}$ ». Le projet est fortement soutenu par les universités et par Jean Pouilloux, alors directeur du département des sciences humaines au CNRS. Dans ces conditions, l'idée d'une Maison créée sous la forme d'un groupement d'intérêt public est accueillie favorablement par le ministère de la Recherche et le CNRS, statut que la nouvelle Maison MRASH a conservé pendant presque dix ans. Par la suite, plusieurs partenaires constitutifs du GIP, dont le CNRS, ayant souhaité se retirer au moment de l'éclatement de la MRASH, les deux entités distinctes qui se sont constituées à Lyon et à Grenoble ont alors perdu l'autonomie conférée par le GIP et sont devenues des instances du type « Unité mixte de service », rattachées directement au CNRS et aux universités concernées.

Dans le cas de Nantes, le projet de Maison est porté là aussi par une personnalité du monde universitaire, Alain Supiot, professeur des universités. Le lien social est le thème fédérateur de la Maison dont l'existence est justifiée ainsi par son fondateur'2 : " l'Homme et les sociétés qui l'instituent sont des réalités trop complexes pour se laisser saisir par une science unique... C'est pourquoi il nous fallait une maison qui réunisse sous un même toit des juristes, des économistes, des linguistes, des psychologues, des sociologues, des géographes, des historiens et d'autres à venir ». La Maison Ange-Guépin,

11 - Maurice Garden, ibid

12 - Alain Supiot, Maison des sciences de l'Homme Ange Guépin, Nantes, Éditions MEMO, 1996, p. 7 
du nom d'un « médecin des pauvres qui, pour comprendre la misère des hommes de son temps se fit successivement historien, sociologue, épidémiologue, et même homme politique ${ }^{13}$ ", constitue un modèle original. Elle n'accueille pas d'unités de recherche mais uniquement des programmes scientifiques sélectionnés par un conseil scientifique propre à la Maison, pour une durée déterminée. Cette Maison a été d'emblée conçue par son initiateur sous la forme d'un GIP. Elle est représentée dans le conseil d'administration de la Maison des sciences de l'Homme de Paris et, réciproquement, l'administrateur de la FMSH est membre de droit du conseil scientifique de la Maison Ange-Guépin.

\section{Les Maisons de la deuxième génération et le statut d'UMS}

Dans les années quatre-vingt-dix, le concept de «Maisons des sciences de l'Homme » séduit des scientifiques fortement investis localement, soucieux de rassembler équipes de recherche et moyens de travail dignes de ce nom et de faire reconnaitre le potentiel de vraies masses critiques de recherche en sciences de l'homme et de la société en dehors de l'lle-de-France. Cet intérêt rencontre celui, grandissant, des instances nationales (CNRS et ministère de la Recherche). Leur conjonction va impliquer pour les futures Maisons de nouveaux avantages et de nouveaux inconvénients.

Avantages, car le CNRS et le ministère ont mené, sur la décennie 1990, une véritable politique de développement scientifique en régions particulièrement bénéfique aux SHS, alors concentrées en lle-de-France à plus de $70 \%$ en ce qui concerne le CNRS. Soutenant les universités les plus volontaristes et avec l'appui des

13 - Alain Supiot, ibid. 
collectivités locales, très désireuses de promouvoir le développement de la recherche sur leur territoire, ils ont été des acteurs institutionnels déterminés pour une politique d'implantation de nouvelles Maisons. Grâce à la convergence d'intérêts entre acteurs nationaux et régionaux, remarquable en elle-même et plus encore dans la durée, les acteurs scientifiques de ces projets ont bénéficié d'importants soutiens financiers pour les mener à bien. Les contrats de plan État-Régions (CPER) ont généralement constitué le cadre de ces partenariats.

Les futures Maisons ont cependant payé ce soutien d'une moindre autonomie, les instances nationales et les instances universitaires étant les unes comme les autres soucieuses de ne pas multiplier les institutions dotées de la personnalité morale, pour des raisons à la fois financières et de fonctionnement institutionnel. Comme nous l'avons souligné à propos de la MRASH, le CNRS par exemple était devenu très hostile à la multiplication des GIP et, au niveau local, les universités étaient peu favorables à la création d'institutions autonomes susceptibles de se développer en concurrence avec ellesmêmes. Les Maisons ont dès lors été définies comme entités rattachées aux universités, à la différence des Maisons plus anciennes. Par ailleurs leur activité de recherche proprement dite n'a paradoxalement pas été explicitement reconnue comme telle, les MSH de cette génération étant constituées par le CNRS sous forme d'Unités mixtes de service ou UMS. II est possible que ce choix ait été dicté par un souci de plus grande souplesse de procédure et pour faciliter la mise en route des Maisons, une fois achevée la période de construction. La création d'une structure de recherche de type fédératif aurait en effet nécessité un examen par le Comité national de la recherche scientifique et possiblement par plusieurs sections pour tenir compte du caractère interdisciplinaire des 
projets. Plus simple à mettre en œuvre, le statut d'UMS constitue principalement un outil de gestion. II implique un pilotage conjoint par le CNRS et l'université de rattachement de la Maison, donc l'octroi de moyens provenant du CNRS et du ministère de la Recherche. Mais il s'agit plus de moyens pour des services communs que pour des programmes scientifiques fédérateurs conduits par la Maison. Le rôle du directeur de l'UMS, qui est aussi le directeur de la Maison, ne s'en trouve pas facilité dans ses relations avec les unités de recherche qui lui sont rattachées, ni dans ses relations avec son (ses) université(s) de rattachement ${ }^{14}$. C'est particulièrement vrai au moment de la préparation du contrat quadriennal de recherche ${ }^{15}$ puisque la Maison n'est généralement pas reconnue comme structure de recherche ${ }^{16}$. Cette situation paradoxale oblige certaines Maisons à présenter leur demande de financement sous la forme de «programmes pluri-formations » (ppf), seules lignes de crédit ouvertes à des financements hors structures de recherche labellisées!

\section{Sur le rôle du CNRS}

Avant de poursuivre l'historique des Maisons, il n'est pas inutile de revenir ici sur le rôle du CNRS dans la définition et le montage des

\footnotetext{
14 - II peut y avoir plusieurs universités de rattachement de la Maison lorsqu'elle abrite des unités ou équipes de recherche de plusieurs universités.

15 - Ce document établit sur une base contractuelle les moyens dont souhaite disposer l'université pour la recherche. Ces moyens sont attribués non pas globalement mais unité par unité, en fonction de leur évaluation, par le CNRS et le ministère de la Recherche s'il s'agit d'unités mixtes ou par le ministère seulement dans le cas contraire, d'où l'importance pour les Maisons d'être reconnues elles-mêmes comme des structures de recherche.
}

16 - Certaines Maisons cumulent le statut d'UMS et de centre de recherche de l'université. C’est le cas à Aix, Poitiers et Caen par exemple. 
projets de nouvelles Maisons entre 1992 et 199417. Le département scientifique SHS a en effet mené à cette période une politique particulière de structuration de la recherche et y a affecté des moyens financiers exceptionnels pour la construction, l'équipement, les postes et le fonctionnement de Maisons qui, en définitive, étaient destinées à être des outils de recherche dans un environnement majoritairement universitaire ${ }^{18}$.

Outre le fait qu'il s'agissait donc d'une structuration au service de la recherche SHS dans son ensemble, cette politique a aussi été innovante. Elle a en effet été accompagnée par le développement d'une culture de projets peu en usage dans les administrations publiques à l'époque. II s'agissait de mettre en place une programmation de moyens sur plusieurs années mettant en jeu différents partenaires, non seulement pour le volet immobilier mais aussi pour les équipements et le fonctionnement, sans oublier les postes ${ }^{19}$. Fondée sur un projet scientifique ${ }^{20}$, chaque opération, après expertise et avis favorable du département scientifique, était validée par la direction générale de l'organisme, validation solennelle qui offrait au chef de projet l'assurance que l'opération pourrait être conduite à son terme avec les moyens nécessaires. Plus que cela même, dans la mesure

\footnotetext{
17 - Voir aussi Bowen John R. et Bentaboulet Martine, (2002), « On the institutionalisation of the Human and social sciences in France », Anthropological Quarterly, 75, p. 537-556.
}

18 - On comptabilise en 200517000 enseignants-chercheurs pour environ 2000 chercheurs CNRS en SHS.

19 - Une grande partie des postes d'ingénieurs, techniciens, administratifs (ITA) pour les Maisons ont été affectés par le CNRS (soit 689 postes ITA CNRS, dont un nombre important de postes de catégories A et B, comparés à 357 postes IATOS non CNRS). Source: enquête réalisée par le conseil des directeurs, 22 avril 2005

20 - Le projet proposé devait être fondé sur une analyse des disciplines mobilisables localement et des perspectives de développement, eu égard au contexte national et international et conduire à terme à une visibilité nationale et internationale de la Maison. 
où les différents services administratifs (du siège et des délégations régionales) étaient requis pour faciliter sa réalisation. Chaque nouvelle Maison a, de cette façon, été dotée d'un dossier d'opération, véritable base contractuelle pluriannuelle et cadre de référence pour les chefs de projet et le département SHS du CNRS. L'exercice pour autant n'était pas facile, dans la mesure où le choix d'un projet et d'une identité scientifique, même concerté avec les universités locales, impliquait d'emblée que certaines équipes ou unités de recherche seraient rattachées à la Maison et que d'autres en seraient exclues. On le voit, la création des nouvelles Maisons n'a pas été sans susciter des difficultés dans les universités. Les projets scientifiques des Maisons de Caen, Poitiers, Aix et Toulouse sont ainsi à l'origine des profils des Maisons actuelles. Caen et Poitiers correspondent à des profils dits « généralistes » en ce sens qu'elles rassemblent des équipes de recherche de différentes disciplines, alors qu'Aix correspond plutôt au profil d'une Maison thématique.

La Maison de la recherche en sciences humaines ou MRSH de Caen, fondée en 1995, regroupe des géographes, des sociologues, des historiens, des littéraires, des psychologues et des bioinformaticiens. Elle affiche de nombreux axes de recherche, identités/ mémoire, traitement des données sociales ${ }^{21}$, questions rurales, modélisation (en sciences cognitives et sciences sociales), sociétés civiles contemporaines, problématiques du risque et un plateau technique constitué par le plan de Rome.

Celle de Poitiers, la Maison des sciences de l'Homme et de la société ou MSHS de Poitiers créée en 1996 regroupe, elle aussi,

21 - C'est cet axe qui constituait à l'origine le volet localisation du projet scientifique pour le CNRS. 
des laboratoires de nombreuses disciplines mais elle a resserré le nombre de ses axes autour de trois priorités ; cognition, langages et communication; mouvements de populations et mutations économiques et sociales ${ }^{22}$; histoire, civilisation et littérature.

La Maison méditerranéenne des sciences de l'Homme ou MMSH à Aix, «née » en 1997, a été conçue pour rassembler en un même lieu une dizaine de gros laboratoires et constituer ainsi une masse critique de recherche sur l'espace « Méditerranée». Elle apparaît comme une Maison thématique organisée en deux pôles, " archéologie et préhistoire » et « sciences sociales et humaines ».

Quant à la Maison de Toulouse, à l'origine Maison de la recherche de Toulouse le Mirail, elle a été conçue en 1994 comme un lieu d'hébergement d'équipes universitaires, d'unités mixtes de recherche et de services administratifs de l'université du Mirail. Par la suite et sur une base volontariste, elle a souhaité renforcer un petit nombre d'axes scientifiques prioritaires et s'ouvrir à une autre université présente à Toulouse, l'université de sciences sociales Toulouse 1, conformément aux principes de la charte des MSH adoptée en 2000. Parmi ses axes de recherche, on peut citer : territoires, villes, mobilités; conditions de vie ; études sur le travail ; sciences humaines et sociales pour l'entreprise ; ou encore interactions, cognition, information.

\section{Les Maisons de la troisième génération, le rôle du Fonds national de la science et du COS}

À partir de 1998 le ministère chargé de la recherche est à nouveau un grand ministère qui réunit l'Éducation nationale et la 
Recherche, sous la responsabilité d'un nouveau ministre, Claude Allègre. Désireux de soutenir fortement la recherche en SHS, celui-ci s'entoure d'un Conseil national de la science et d'un Conseil national pour le développement des sciences humaines et sociales qui l'encouragera fortement à soutenir une politique d'essor des Maisons des sciences de l'Homme et appuiera le projet de réseau entre Maisons.

De nombreuses personnalités dans l'entourage du ministre sont acquises à la politique des Maisons des sciences de l'Homme. Alain Supiot, fondateur de la Maison des sciences de l'Homme de Nantes, est président du CNDSH. Robert Ilbert, professeur des universités, chargé de mission pour la recherche dans un précédent ministère, est conseiller pour la recherche SHS avec Antoine LyonCaen auprès du directeur de la recherche. Robert Ilbert a été chef de projet pour la Maison méditerranéenne des sciences de l'Homme à Aix et en est le directeur depuis sa création en 1997. Maurice Garden, fondateur de la MRASH à Lyon, a été responsable pour les sciences humaines à la Direction de la recherche et des études doctorales dans un ministère précédent.

Il est membre du cabinet du ministre jusqu'en 1999, puis chef de la Mission scientifique universitaire chargée de la contractualisation de l'ensemble des universités française ${ }^{23}$. Éric Espéret, chef de projet puis directeur de la Maison de Poitiers, deviendra président de l'université de Poitiers et sera aussi chargé d'une mission pour une « Nouvelle définition des tâches des enseignants et des enseignants-chercheurs dans l'enseignement supérieur français » auprès du ministère en 2001.

23 - II sera aussi le directeur de l'Action concertée incitative « réseau des MSH » 
C'est ainsi qu'à la fin des années 1998-1999 et au début des années 2000, les conditions du développement de la recherche en sciences humaines et sociales au ministère vont connaître un essor important. D’une part, un plan de croissance des universités dit « Université du troisième millénaire U3M » est mis en chantier. II ne concerne bien sûr pas que les SHS mais elles seront aussi bénéficiaires de ce nouvel élan. D'autre part, le ministère ${ }^{24}$, souhaitant piloter de manière volontariste une recherche sur programmes sans se limiter au soutien traditionnel des équipes et unités de recherche labellisées, crée le Fonds national de la science. Plusieurs programmes ou « Actions concertées incitatives ou $\mathrm{ACl} »$ seront ainsi soutenus en SHS, dont un programme spécifique pour le développement des Maisons des sciences de l'Homme, l'ACl réseau des MSH créé en 1999. Comme toutes les actions concertées incitatives du FNS, l'ACI réseau des MSH est dotée d'un directeur et d'un conseil scientifique. Dans cette $\mathrm{ACl}$ particulière, le directeur est assisté par un comité de pilotage $\mathrm{e}^{25}$ afin de permettre une coordination entre les politiques du département SHS du CNRS, de la Direction de la recherche et de la Mission scientifique universitaire du ministère, ces trois instances siégeant dans le comité de pilotage.

La nécessité, pour la Direction de la recherche au ministère, de clarifier les principes fondateurs des Maisons des sciences de l'Homme et de définir leurs missions s'est imposée dès 1998. Après consultation du conseil scientifique de l'ACl et avis des directeurs des $\mathrm{MSH}$, une charte des Maisons des sciences de

\footnotetext{
24 - Le terme "ministère » recouvre en fait différents acteurs au sein du ministère, sans nécessairement de lien de subordination entre eux, ce qui conduit parfois à des prises de positions multiples, mal coordonnées voire contraires, et donc difficiles à comprendre pour un interlocuteur extérieur qui croit s'adresser à un acteur unique. 
l'Homme est adoptée. À la signature de la convention créant le Réseau des Maisons des sciences de l'Homme en 2000, le conseil scientifique de l'ACl est transformé en conseil d'orientation stratégique du réseau ${ }^{26}$. Cette convention crée un cadre institutionnel nouveau dans lequel vont s'inscrire non seulement l'expertise des projets scientifiques réalisés dans le cadre du réseau, mais aussi la validation des projets de nouvelles MSH par le conseil d'orientation du réseau, ainsi que le prévoit le texte de la convention.

Or de nombreux projets de nouvelles Maisons sont candidats à une validation pour accéder à l'entrée dans le Réseau des Maisons des sciences de l'Homme. Les projets de Maisons des sciences de l'Homme sont en effet encouragés par le ministre Claude Allègre, fortement désireux de voir se développer ce type de structuration de la recherche en SHS sur l'ensemble du territoire, en synergie avec les objectifs du plan $\mathrm{U}^{3} \mathrm{M}^{27}$.

Les projets de Strasbourg, Paris-Nord, Nice et Lille sont les premiers de cette troisième génération de Maisons des sciences de l'Homme à être présentés devant le conseil d'orientation du Réseau. Les chefs de projet, nommés par le ministère ${ }^{28}$, sont accompagnés des présidents des universités concernées,

26 - Un glissement sémantique le transformera en conseil d’orientation scientifique du réseau au fil du temps.

27 - Le plan « Université du troisième millénaire ou U3M » est destiné à servir de cadre au développement des universités à partir de l'an 2000. La construction et l'augmentation des surfaces d'enseignement mais aussi de recherche sont prévues et inscrites dans les contrats de plan État-Régions 2000-2007. Pour ces opérations, le ministère a institué une procédure avec un dossier d'opération calqué sur celui mis en place par le CNRS pour les opérations de localisation inscrites dans les contrats de plan précédents.

28 - Et non par le CNRS, comme ils l'avaient été dans la deuxième génération des Maisons. 
témoignant de la concertation autour du projet au sein des établissements universitaires.

La Maison internationale des sciences de l'Homme d'Alsace ou MISHA à Strasbourg a dû, pour s'imposer, affronter une situation inédite compliquée en raison de l'existence de la précédente Maison des sciences de l'Homme, très peu active au plan de la recherche. Les scientifiques des universités strasbourgeoises comme les collectivités locales étaient donc peu enclins à soutenir un nouveau projet. Le chef de projet choisi par le ministère en concertation avec le CNRS, un professeur de l'université de Strasbourg 3, spécialiste d'histoire et de l'antiquité, a dû mener une consultation souvent ardue auprès de ses collègues professeurs, avant de proposer une ébauche de thèmes de recherche partagés, susceptibles d'être portés par la future Maison. II est en effet très difficile, pour un scientifique, de convaincre des collègues d'autres disciplines de participer à une réflexion sur une approche pluridisciplinaire d'objets de recherche, dont le choix reste à préciser par les chercheurs eux-mêmes.

L'exercice demande beaucoup de temps, de conviction ${ }^{29}$ et de diplomatie, surtout lorsque l'identité scientifique de la future Maison n'est pas prédéfinie. En l'occurrence pour Strasbourg, le noyau dur du projet sera progressivement organisé autour de quatre axes: Europe, cultures, épistémologie des savoirs et sciences de l'antiquité.

Le cas de la Maison des sciences de l'Homme dite MSH ParisNord est pour sa part exemplaire en matière de construction d'un

29 - La participation de représentants de la Direction de la recherche et du directeur de l'ACl réseau des MSH aux réunions organisées par le chef de projet a parfois été nécessaire pour mobiliser les universitaires autour du projet dans sa phase de définition. 
projet intellectuel par allers-retours successifs et concertations à tous les niveaux, qu'il s'agisse de celles menées par le chef de projet avec les responsables des équipes de recherche au sein des universités Paris 13 et Paris 8, les plus directement concernées par le projet, ou de celles menées auprès des collectivités locales pour les associer le plus en amont possible au projet, ou, enfin, de la concertation menée au niveau national avec la Direction de la recherche et le $\mathrm{CNRS}^{30}$.

L'exercice visant à définir l'identité scientifique de la Maison autour de projets pluridisciplinaires était ici rendu plus facile dans la mesure où deux axes de recherche avaient été fixés dès l'origine par le ministère. Selon les termes de la lettre de mission du chef de projet, ils sont ainsi définis : "l'un associera les SHS, les industries culturelles et l'art (notamment les domaines de l'image et du son), l'autre les SHS et la santé ». Ces axes tenaient compte à la fois du potentiel de recherche mobilisable dans le Nord francilien et de l'existence de domaines de recherche peu structurés ou en cours de structuration au niveau national. L'ambition du ministère était surtout de doter cette partie du territoire d'lle-de-France d'un grand centre de recherche en sciences de l'homme et de la société, d'accroître le rayonnement des universités présentes localement et, par effet induit, l'attractivité du territoire de Seine-Saint-Denis.

D'une façon générale, s'agissant des projets de Maisons émergentes, on observe que les conditions mises à leur validation constituent un défi pour les équipes, localement : car ces projets représentent une opportunité à saisir pour bénéficier de conditions de travail et de moyens nouveaux, mais impliquent aussi de multiples contraintes pour définir des programmes de recherche qui mobilisent des cher-

30 - On pourra utilement consulter le dossier de faisabilité de la Maison, établi en décembre 2000 par le chef de projet Pierre Moeglin, professeur des universités, à la demande du ministère et disponible en ligne sur le site de la MSH Paris-Nord. 
cheurs de plusieurs disciplines et d'universités différentes, le cas échéant. II n'est pas rare que comme à Lille, Montpellier ou encore Rennes, les projets fassent l'objet de plusieurs ébauches et allers-retours avant d'obtenir une expertise favorable.

\section{Sur le rôle des collectivités locales}

Les collectivités locales jouent un rôle déterminant dans la politique d'implantation des MSH car elles sont très désireuses de favoriser la visibilité de la recherche sur leur territoire et savent peser de leur influence pour faire aboutir les projets. Les régions - mais aussi les départements, les municipalités et les groupements de communes - sont fortement sollicités pour leur appui dans les différentes phases. Elles peuvent aussi représenter un moyen de recours (ou de pression) lorsque des projets sont en difficulté au niveau national ou local.

Fortement sollicitées bien sûr pour le financement des opérations, elles disposent de moyens financiers qui, souvent décisifs, représentent une part importante du budget global, aussi bien pour l'immobilier - avec le cas échéant l'apport du terrain, comme pour la MSH Paris-Nord à Saint-Denis -, que pour les équipements. Mais l'appui des collectivités ne se fait pas sans contrepartie, car celles-ci sont de plus en plus désireuses d'être partie prenante des choix et des orientations en ce qui concerne les programmes scientifiques. Dans certaines régions comme en Rhône-Alpes et en Pays de la Loire, il existe un conseil de la recherche. Suite aux appels d'offres régionaux, les programmes scientifiques sont évalués par des experts scientifiques et les financements régionaux attribués aux projets sélectionnés. Dans d'autres régions, le conseil régional a pu être tenté, comme à Lille, d'imposer ses choix en tentant d'infléchir les orientations scientifiques de la future Maison. 
Le « localisme » ou le « régionalisme » sont de fait des dangers inhérents aux projets de Maison des sciences de l'Homme. Les conseils scientifiques propres à chaque Maison et le conseil scientifique du Réseau restent vigilants sur ces questions. On peut par ailleurs penser que le rôle des collectivités sera déterminant à l'avenir pour le rayonnement de la recherche en général et des Maisons en particulier, dans la mesure où elles peuvent mettre en place des « chaires régionales » pour des chercheurs invités et des bourses d'accompagnement pour des jeunes chercheurs ou étudiants chercheurs. De tels dispositifs faciliteraient une politique scientifique des Maisons en cohérence avec leur identité scientifique, identité qu'elles se doivent de construire à l'échelle nationale, européenne et internationale.

\section{Sur l'historique du Réseau}

L'idée d'un réseau entre les structures de type MSH est pratiquement aussi ancienne que les Maisons elles-mêmes. Fernand Braudel, en effet, en créant la Fondation de la Maison des sciences de l'homme boulevard Raspail, avait prévu et organisé des interactions institutionnelles entre la Maison de Paris et celles de Bordeaux et Strasbourg, créées dans le même mouvement historique. Les responsables des Maisons qui se sont développées ultérieurement à Nantes (Maison Ange-Guépin) et à Lyon (Maison Rhône-Alpes des sciences de l'Homme), puis à Caen et Aix, partageaient ce même souci de développer des liens privilégiés entre leurs institutions ou futures institutions, comme en atteste l'existence de réunions en ce sens dès 1992 ${ }^{31}$. L'idée est reprise en 1998 par les directeurs des Maisons officiellement créées, qui organisent une réunion à Poitiers pour un tour de table.

31 - Réunion organisée par Maurice Aymard, administrateur de la Maison des sciences de l'Homme à Paris. 
La dynamique des acteurs locaux alliée à la présence concertée au niveau national de responsables acquis à l'intérêt du Réseau est déterminante. La Direction de la recherche, en la personne de Robert Ilbert, et le Conseil national pour le développement des sciences de l'Homme, présidé par Alain Supiot, recommandent la mise en réseau des Maisons, en tant qu'outil stratégique pour développer leurs échanges scientifiques et promouvoir la mutualisation des moyens techniques dont elles disposent. Suzanne Srodogora ${ }^{32}$ et Maurice Garden, alors au cabinet du ministre, appuient cette proposition et en 1999 l'ACl «Réseau des MSH » est créée.

\section{Les années 1999-2001 de l'ACI}

Lidée de Réseau, bien que présente depuis plusieurs années, n'est pas facile à traduire dans les faits. Elle fait appel à plusieurs concepts : maillage du territoire, coopérations scientifiques, partage et échange de ressources... Encore faut-il connaître les forces et faiblesses des Maisons appelées à constituer le Réseau. D'où l'état des lieux effectué par la Direction de la recherche en 1999 et les premières priorités de l'ACI.

En concertation avec la Direction de la recherche, la MSU et le CNRS, les instances de l'ACI proposeront une série d'actions pour mettre à niveau les équipements des Maisons (fonds documentaires, catalogage, informatique...), améliorer l'accueil des doctorants et créer les conditions de travail pour des programmes de recherche conduits par la Maison. Ces moyens feront l'objet de négociations et d'arbitrages sur la base des contrats d'objectifs demandés aux directeurs des Maisons par les instances de l'ACl. Dès les premières années,

32 - Suzanne Srodogora a été secrétaire générale du département scientifique SHS du CNRS dans la période 1994-1998. 
ces actions structurantes sont mises en œuvre et mobilisent des financements importants au regard des subventions dont disposent annuellement les Maisons. Pendant les trois premières années de l'ACl, les financements au bénéfice des $\mathrm{MSH}^{33}$ ont ainsi été multipliés par 1,7 environ, grâce au Fonds national pour la science. Cette formidable "bouffée d'oxygène » a représenté un saut qualitatif dans les financements et a permis d'accélérer la mise à niveau et le renouvellement des équipements. Les autres actions entreprises ont surtout visé à aider les différentes Maisons à développer leur identité scientifique et à favoriser interdisciplinarité et ouverture internationale, deux objectifs retenus pour l'ensemble des Maisons et le Réseau (voir infra).

Sur le plan des effectifs, les Maisons concernent environ 4000 chercheurs et enseignants-chercheurs sur un total de 20000 chercheurs et enseignants chercheurs SHS (17000 universitaires, 2000 chercheurs CNRS et 1000 chercheurs d'autres organismes).

\section{La charte et les statuts du Réseau}

Afin de créer les bases du Réseau, il était nécessaire d'expliciter les objectifs partagés par les Maisons. Une « charte des MSH », rédigée à l'initiative de l'ACI sur les bases de textes antérieurs et de nouvelles propositions, est soumise à l'ensemble des directeurs ${ }^{34}$. Une réunion est organisée en 1999 au ministère de la Recherche

\footnotetext{
33 - Dans cette estimation ne sont comptabilisés que les financements attribués aux MSH, UMS et autres structures (sauf la Fondation MSH), et non ceux des unités de recherche qui leur sont rattachées.

34 - Outre les directeurs ayant participé à la réunion de Poitiers en 1998, sont présents par exemple les directeurs de la MOM à Lyon, de la Maison archéologie et ethnologie à Nanterre, de la Maison des sciences de la ville à Tours et de la MISHA, future Maison de Strasbourg.
} 
pour en débattre et, dans l'année qui suit, un accord se fait sur le texte de la Charte $^{35}$.

Le Réseau doit par ailleurs être doté de statuts pour assurer son existence pérenne. Les discussions sur ce sujet sont difficiles, car si l'ensemble des directeurs de Maisons est favorable à un statut conférant l'autonomie morale et juridique au Réseau, les instances de tutelle sont sur ce point plus réservées. Par ailleurs, aucune des structures juridiques existantes n'est satisfaisante. La création d'une fondation est un processus lourd qui nécessite des capitaux importants et des partenaires privés aux côtés des acteurs publics. À l'issue d'un jeu délicat de tensions entre la Maison de Paris et les Maisons de province, l'idée d'utiliser la Fondation Maison des sciences de l'Homme pour structurer le réseau, par exemple en élargissant la fondation «parisienne» en une fondation « nationale » qui aurait constitué la base juridique du Réseau est éludée. Le statut d'association loi 1901 pose le problème de l'évaluation scientifique et le statut de groupement d'intérêt public, celui de la durée limitée du groupement.

Après deux ans d'efforts infructueux pour parvenir à une solution, un statut minimum est adopté sous la forme d'une convention qui officialise l'existence du Réseau et définit les instances qui en assurent le fonctionnement, conseil de direction et conseil d'orientation stratégique, $\operatorname{COS}^{36}$. La charte annexée à la convention fixe les principes fondateurs des MSH. Elle servira de texte de référence pour l'expertise et la validation des projets des nouvelles Maisons. La convention, signée

35 - Cette charte deviendra la charte du Réseau des MSH.

36 - Les membres du COS ont été désignés par la Direction de la recherche en concertation avec les directeurs des Maisons, la Mission scientifique universitaire et le CNRS. 
en 2001 par le CNRS, le ministère de la Recherche, les Maisons dotées de la personnalité morale et les universités auxquelles sont rattachées les autres Maisons, ne confère pas au Réseau l'autonomie morale et juridique. Prévue pour une durée de quatre ans, elle sera remplacée par un nouveau dispositif à partir de 2006.

\section{De nouvelles perspectives pour le Réseau}

Le nouveau dispositif dote le Réseau d'un statut de groupement d'intérêt scientifique, structure dénuée de la personnalité morale. Les principales modifications par rapport à la convention précédente portent sur le fait que le ministère de la Recherche n'est plus signataire et sur les instances du Réseau. Outre le nouveau mode de fonctionnement que le Réseau aura à expérimenter en raison de ces changements, le problème crucial, à partir de 2006, est celui du financement des actions à poursuivre et celui des actions nouvelles. La disparition du FNS et la création de l'ANR créent en effet un contexte très différent pour le Réseau encore fragile. Ce peut être aussi une chance. La réflexion sur les choix stratégiques du Réseau sera déterminante pour son avenir et pour son financement ${ }^{37}$.

37 - Les nouvelles fondations à caractère scientifique, annoncées dans la loi d'orientation et de programmation de la recherche et destinées en premier lieu à animer des campus à vocation scientifique, pourraient constituer une piste pour l'avenir du Réseau, sorte de campus scientifique à localisations multiples. Parallèlement, un rapprochement avec d'autres institutions SHS en France (instituts d'études avancées par exemple) et en Europe pourrait permettre d'élargir les échanges au sein du Réseau. 
Histoire d'une structuration de la recherche:

de la Maison au Réseau 
Avenir de la recherche 


\section{3 \\ Les principes fondateurs du Réseau national des Maisons des sciences de l'Homme}

Pierre Guibentif I

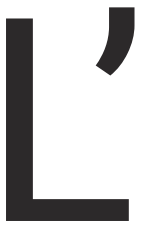

objectif du présent chapitre est de proposer une synthèse de l'état actuel de la réflexion du Réseau national des Maisons des sciences de l'Homme sur les principes orientant ses activités. II prend appui sur deux types de documents. D'une part, un ensemble de textes - aussi bien des textes normatifs que des travaux d'analyse - dont la production a jalonné le processus de constitution du Réseau. D’autre part, un ensemble de rapports d'activités des Maisons des sciences de l'Homme. II s'agit à la fois de rappeler, de manière dûment documentée, quelles ont été les visées du Réseau, dans l'esprit de ceux qui ont participé à sa fondation et à sa promotion ultérieure, et d'examiner comment ces visées ont été concrètement perçues et opérationnalisées au niveau des MSH constituant le Réseau.

Cet objectif s'inscrit dans le cadre de la mission du conseil d'orientation scientifique du Réseau, telle que définie dans la convention de partenariat qui est à la base du Réseau, selon laquelle le COS 
« formule un avis sur les orientations générales et les priorités du Réseau ». La méthode retenue ici a consisté à valoriser, dans la préparation de cet avis, l'expérience historique du Réseau et les pratiques actuelles des MSH.

Il existe des rapports étroits entre le présent travail et celui, animé dans le cadre du Réseau par Linda Hantrais, sur le thème de l'évaluation. Une formulation précise des "orientations générales et priorités » est en effet nécessaire à la définition des «critères d'évaluation $»^{38}$. Dans le processus de réflexion engagé par le COS, les deux thèmes ont cependant, à juste titre, été considérés comme méritant d'être approfondis dans un premier temps de manière autonome. Ceci permet, dans la réflexion sur les orientations, de bien prendre en compte les particularités de la dynamique actuelle des sciences de l'homme et de la société, et de considérer non seulement les réalisations actuelles, mais également les aspirations et préoccupations. Et, dans la réflexion sur l'évaluation, il est possible de tirer pleinement parti des avancées des méthodes et techniques d'évaluation dans des domaines divers, et de reformuler les principes applicables spécifiquement au Réseau en fonction des nécessités méthodologiques de l'évaluation des activités en cours, sans que cela risque d'entraîner une limitation dans la discussion des développements à venir.

\section{Les principes fondateurs dans les textes}

\section{La doctrine des trois«i»}

Le document à considérer ici en première ligne est la Charte des Maisons des sciences de l'Homme, à laquelle adhèrent les

38 - Voir ci-après le chapitre "Enjeux de l'évaluation des Maisons des sciences de l'Homme». 
institutions membres du Réseau ${ }^{39}$. Ce document a été élaboré en 1999 en concertation entre les directeurs des douze MSH fondatrices du Réseau et les groupes de réflexion animés par la Direction de la recherche du ministère de la Recherche, et adopté le 5 juin 200040,41. Il figure en annexe de la Convention de partenariat qui fonde le Réseau, signée le 9 août 2001 entre les ministères de l'Enseignement supérieur et de la Recherche, le CNRS, et les MSH existant à ce moment-là, représentées soit par leurs établissements de rattachement, soit par les organismes les constituant.

La traduction de la charte, dans la pratique du suivi des MSH existantes et de l'évaluation des propositions de nouvelles maisons, a conduit à la formulation de ce que l'on a pu appeler la « doctrine des trois i $»^{42}$. Selon celle-ci, trois « ambitions » caractériseraient le

39 - Ce document (devenu charte du Réseau des MSH) est accessible sur le « portail du Réseau » (http: //www.msh-reseau.prd.fr/).

40 - Garden Maurice, (2003), "De la Maison des sciences de l'Homme de Fernand Braudel au Réseau national des Maisons des sciences de l'Homme», p. 13 s. et Rouillard Pierre, (2003), "Les Maisons émergentes », p. 22, Sciences de l'homme et de la société. Lettre du département, $n^{\circ}$ 68, numéro spécial : Les structures fédératives ( $\mathrm{Pa}$ ris, CNRS), août 2003.

41 - Le projet d'une charte et d'un réseau des MSH est déjà à l'ordre du jour d'une rencontre de septembre 1992 entre les MSH alors existantes ou en cours de création. Un premier texte est proposé en 1994 (Bowen John R. et Bentaboulet Martine, (2002), " On the institutionalisation of the Human and social sciences in France », Anthropological Quarterly, 75, p. 537-556).

42 - Pollet Gilles (2003), «Le Réseau des Maisons des sciences de l'Homme : un dispositif au service de l'aménagement du territoire de la recherche en SHS », Sciences de I'homme et de la société. Lettre du département n 68 - numéro spécial : Les structures fédératives (Paris, CNRS), août 2003, p. 17-18; Commaille Jacques, Rouillard Pierre (2004), Les maisons des sciences de l'homme dans les sciences humaines et sociales, p. 6, Paris, 16 juin, note communiquée à Édouard Brézin, 15 p. ; Rouillard Pierre, (2004), "Le réseau national des Maisons des sciences de l'Homme », in : (coll.), Le réseau national des MSH, p. 5. 
réseau: l'interdisciplinarité, une dynamique interinstitutionnelle et l'orientation vers l'international.

Ces trois principes s'expriment en effet en divers endroits de la charte:

\section{- interdisciplinarité :}

art. 1 : «les MSH [...] regroupent [...] des chercheurs (et) des équipes [...] de différentes disciplines »;

art. 2 : «Elles développent [...] des programmes de recherche communs à plusieurs disciplines [...]»;

art. 3 : «Le réseau favorise la création [...] de "laboratoires" sans murs. »;

\section{- dynamique interinstitutionnelle :}

art. 1 : «Les MSH [...] regroupent [...] des unités de recherche dépendant de différentes institutions »;

art. 2 : «Elles développent [...] des programmes de recherche communs à plusieurs [...] unités de recherche » ;

art. 3 : «Elles sont reliées entre elles et forment un réseau de communication [...]»;

art. 4 : «Elles disposent d'instruments de travail communs aux différentes unités [...]» ;

\section{- orientation vers l'international :}

art. 5: «Elles favorisent l'insertion des chercheurs dans la communauté internationale [...]» ;

art. 6 : « (elles) veillent à la meilleure diffusion possible de leurs recherches [...] à l'échelle locale, nationale et internationale. »

L'utilité d'une charte des Maisons des sciences de l'Homme avait déjà été soulignée, quelque temps avant l'adoption de ce texte, par le rapport Pour une politique des sciences de l'homme et de la 
sociétét3. Ce texte énonçait un ensemble de principes qu'une telle charte devrait inclure ${ }^{44}$, en commençant par l'interdisciplinarité, suivie de la « mise en œuvre du partenariat CNRS/Université » - un des fondements de la vocation interinstitutionnelle des MSH - et de l' " orientation internationale ». Les conclusions de ce même rapport insistaient encore sur la nécessité du « dialogue entre les disciplines» et sur le fait que le réseau des MSH devait être « ouvert sur l'international $»^{45}$.

Les trois « $\mathrm{i}$ » sont également consacrés par la convention signée quelque temps après l'adoption de la charte. Le préambule de ce document les réunit en une formule synthétique, selon laquelle le Réseau doit contribuer à «augmenter les échanges interdisciplinaires et internationaux tout en permettant de meilleures synergies entre les établissements et organismes $»^{46}$. L'article 3 , concernant les «Objectifs et missions du réseau », consacre un alinéa à chacun des trois « $i$ », en énonçant les trois missions suivantes:

- «Contribuer à renforcer les relations [...] entre les SHS et les autres secteurs scientifiques, qui s'inscrivent dans l'objectif général d'interdisciplinarité, d'internationalisation et de décloisonnement des disciplines. » (Accent mis sur l'aspect interdisciplinaire);

\footnotetext{
43 - Conseil national du développement des sciences humaines et sociales (sous la présidence d'Alain Supiot) (2001), Pour une politique des sciences de l'homme et de la société, p. 201 s., Paris, PUF (Quadrige 354).

44 - Ibid., p. 202

45 - Ibid., p. 226 s.

46 - Dans la terminologie de la convention, les « établissements » sont les établissements de rattachement de MSH non dotées d'une personnalité juridique propre, et les « organismes », les organismes constituant eux-mêmes une MSH.
} 
- «Faciliter et favoriser les échanges scientifiques de toute nature entre les signataires » (aspect interinstitutionnel) ;

- «Euvrer en faveur de l'ouverture et de la visibilité internationales des recherches en SHS [...] » (aspect international).

\section{Des trois «i» aux cinq «i»}

L'importance des trois « $i$ » dans l'orientation des activités du Réseau est donc clairement établie. Une réflexion plus approfondie sur le fonctionnement du réseau conduit cependant à reconnaître une importance égale à deux autres principes. Ceci pourrait conduire à énoncer une doctrine des cinq « i ».

Un «quatrième i » est celui du principe d'« implantation régionale ${ }^{47}$, ou encore, pour reprendre un concept proposé dans le cadre plus général des vocations des sciences humaines et sociales : «i » pour « inscription politique et sociale $»^{48}$.

Ce principe s'exprime clairement à l'article 6 de la Charte : «Les $\mathrm{MSH}$ veillent à la meilleure diffusion possible de leurs recherches, auprès des institutions publiques ou privées, à l'échelle locale, nationale ou internationale. »

Il est également lié à deux notions plus abstraites, qui ne se réduisent sans doute pas à des éléments constitutifs de cette « inscription/implantation » mais qui la favorisent puissamment, étant permis d'admettre que c'est bien pour ce motif qu'on y insiste tant. L'une est celle de «lieu identifiable» (article 1 de la charte), qui correspond à l'idée de «lisibilité et visibilité » que les

47 - 47 Rouillard Pierre, (2004), « Le réseau national des Maisons des sciences de I'Homme », in : (coll.), Le réseau national des MSH, p. 4-6.

48 - Pour une politique des sciences de l'homme et de la société, (2001), op. cit., p. 142 
MSH conféreraient à la recherche en sciences sociales ${ }^{49}$. Très explicite sur ce point, Godelier ${ }^{50}$ constate qu'un « avantage » des $\mathrm{MSH}$ est «la production d'une visibilité qui permet le rapprochement de la recherche en SHS et des collectivités locales et des institutions publiques ou privées ». L'autre est celle d' "ancrage» que l'on trouve déjà dans le rapport Pour une politique des sciences de l'homme et de la société ${ }^{51}$. Selon celui-ci, l'un des objectifs des MSH serait « une orientation internationale, avec ancrage dans la région d'accueil ». Cette notion ressurgit dans l'énoncé des « politiques de site » du département des sciences de l'homme et de la société52: par les MSH, le travail en SHS se trouverait «ancré en des lieux précis, dûment matérialisés ${ }^{53,54}$, et se retrouve dans la présentation du Réseau, qui définit les Maisons comme «lieu forum $»^{55}$. Dans le même sens, le texte de présentation du Réseau placé sur le site web de la MSH Paris rappelle que les Maisons sont «nées dans des contextes locaux différents et ancrées dans un tissu régional particulier ».

\footnotetext{
49 - Garden Maurice, (2003), op. cit., p. 15.

50 - Godelier Maurice, (2001), Létat des sciences de la société en France et leur rôle dans la construction de l'espace européen de la recherche, p. 48, Paris, rapport au Premier ministre (publication postérieure à La Documentation française).
}

51 - Pour une politique des sciences de l'homme et de la société, (2001), op. cit., p. 202.

52 - Département des sciences de l'homme et de la société, (2000), «Les orientations stratégiques du département des sciences de l'homme et de la société », Lettre du département (Paris, CNRS), juillet 2000.

53 - "Les structures fédératives : un essai de présentation », Sciences de l'homme et de la société. Lettre du département $n^{\circ} 68$, numéro spécial : Les structures fédératives (Paris, CNRS), août 2003, p. 8.

54 - Cette notion doit être rapprochée du «principe d'unité du site » ( Les structures fédératives... », 2003, p. 8 et 10). Celui-ci vise cependant surtout le rapprochement entre les chercheurs et entre les équipes, au bénéfice en particulier de l'interdisciplinarité, et de la rationalisation de l'usage des équipements.

55 - Rouillard Pierre, (2004), op. cit., p. 4. 
Plus encore que les trois précédents, ce principe peut être directement rattaché à la dénomination «Maison des sciences de l'Homme » elle-même. En effet, le terme « Maison » met l'accent sur la matérialité du lieu qui accueille les activités, lieu singulier et visible; un point sémantique d'ailleurs relevé dans le préambule de la charte, où il est question de « regroupement en un lieu donné (une maison) de chercheurs, équipes ou unités de recherche $»^{56}$.

À propos plus particulièrement de la vocation du réseau, il est question de l'objectif que devrait se donner toute MSH « d'affirmer et de développer une identité scientifique propre $»^{57}$, « de dessiner dans le Réseau un profil scientifique qui en fasse un pôle reconnu nationalement et internationalement $»^{58}$. Cette visée s'exprime dans le préambule de la charte, selon lequel une caractéristique des MSH serait « la définition d'une identité scientifique - avec une ou plusieurs dominantes - qui (lui) donne sa cohérence[...]», ainsi que dans son article 2, selon lequel les MSH « développent chacunes des activités de recherche propres qui contribuent à définir leur identité scientifique. » Nous sommes ainsi conduits à un « cinquième $i$ », pour « identité scientifique ».

\section{Principes complémentaires}

Au-delà des cinq «i » ainsi identifiés, d'autres références d'orientation sont mentionnées, encore que plus brièvement, dans les documents analysés.

\footnotetext{
56 - Cette notion se perd dans la traduction de l'expression « Maison des sciences de l'Homme » adoptée dans la version anglaise de la plaquette de présentation du réseau (Le réseau / National Network..., 2004) : "Social Sciences and Humanities Research Institute ». Significativement, Bowen J. et Bentaboulet M., (2002), dans leur article " On the Institutionalization of the Human and Social Sciences » maintiennent le terme français de «maison » (Anthropological Quarterly, 75, p. 537-556).

57 - Godelier Maurice, (2001), op. cit., p. 47.

58 - Rouillard Pierre, (2004), op. cit., p. 5
} 
La plus générale, énoncée en bonne place dans la convention (article 3, premier alinéa), est la mission de «dynamiser la recherche en sciences humaines et sociales", en visant une «meilleure qualité des résultats obtenus ${ }^{59}$. Cet objectif, implicite dans tous les autres, et qui se traduit lui aussi dans la désignation des institutions en cause, va, si l'on peut dire, de soi. II invite cependant à un constant effort de réexplicitation et de réappréciation des principes plus spécifiques dégagés par la pratique, travail auquel la présente note entend d'ailleurs contribuer.

Une vocation des MSH est l'« articulation entre enseignement doctoral et recherche $\aleph^{60}$, vocation que la charte énonce en ces termes : "(les MSH) soutiennent la formation des jeunes chercheurs, notamment au travers des Écoles doctorales » (article 2).

Une autre vocation est la promotion, dans les SHS, d'une culture scientifique basée sur le travail collectif. Les MSH seraient au service d'une «recherche [...] davantage collective » ${ }^{61,62}$. Dans ce sens, la charte fait plus fréquemment référence aux « équipes et unités » (cinq mentions) qu'aux « chercheurs » (deux mentions). Une exigence à laquelle doit correspondre le fonctionnement des MSH est le « renouvellement » des chercheurs et des programmes (charte, article 2, deuxième section ; « Les structures fédératives... », 2003, op. cit., p. 4).

59 - «Les structures fédératives: un essai de présentation », (2003), op. cit., p. 11.

60 - « ibid » p. 4.

61 - «ibid » p. 5, 7-8.

62 - Sur la mutation du travail en SHS que les MSH entendent ainsi favoriser, voir aussi Pour une politique..., 2001, p. 30 : "Linscription progressive des individus dans des équipes de recherche a mis à mal le culte de "solitude fertile" propre à l'homme de lettres. II n'est plus rare désormais que le progrès des connaissances soit l'œuvre de collectifs plutôt que celle de "grands auteurs". » 
Pour clore cette énumération, rappelons l'objectif de mutualisation des outils, lié à la priorité économique de meilleure planification des investissements en matière d'instrumentation scientifique ${ }^{63}$ et l'objectif de maillage du territoire qui orientent l'activité du Réseau ${ }^{64}$.

Dans les lignes qui suivent, nous nous limiterons aux cinq « $i$ », c'est-à-dire aux principes qui concernent le plus directement le Réseau en tant que tel. Ils le concernent doublement. D'une part, comme pour les autres principes abordés, le Réseau peut utilement contribuer à une réflexion sur leur mise en œuvre et, moyennant le développement d'outils appropriés, à l'évaluation de celle-ci. Mais, bien plus que dans le cas des autres principes, le Réseau est un acteur privilégié de cette mise en œuvre.

Cela est particulièrement évident pour ce qui concerne la dynamique interinstitutionnelle, dont le Réseau est l'un des instruments, ainsi que pour l'identité scientifique des différentes MSH, qui gagne à se définir dans un processus de progressive prise de conscience des contributions - d'ailleurs susceptibles d'évoluer dans le temps - des différentes institutions membres du Réseau.

Les efforts d'internationalisation ont, quant à eux, plus de chance de succès s'ils sont relayés et amplifiés par une structure d'envergure nationale.

Enfin, les exigences d'interdisciplinarité et d'inscription dans la cité concernent sans doute en première ligne les MSH prises individuellement. Mais elles posent des problèmes tels que leur traitement a tout à gagner d'un partage des expériences des différentes Maisons,

63 - «Les structures fédératives : un essai de présentation », (2003), op. cit., p. 9. 64 - Rouillard Pierre, (2004), op. cit., p. 5. 
acquises dans des contextes institutionnels, disciplinaires et socio-politiques variés. Par ailleurs, ce qui est plus important encore, le Réseau ouvre un espace de coopération interdisciplinaire par définition plus vaste que celui que constituent les institutions membres. Et l'inscription dans la cité gagne à ne pas être un simple rapport local entre une Maison et son lieu, mais plus largement, entre un type d'institution et la société, à l'échelle nationale.

\section{Les principes fondateurs en action}

La présente section est basée principalement sur les rapports qui ont été remis par les MSH au COS, dans le cadre de la préparation des réunions des 23 et 24 mai 2005. Cette documentation a été complétée par la consultation des présentations individuelles des $\mathrm{MSH}$, contenues dans des publications concernant les MSH en général ${ }^{65,66}$, ainsi que des sites web des Maisons. En l'état, il ne s'agit pas d'une comparaison systématique, où seraient inventoriées, pour chaque point abordé, toutes les prises de positions ou mesures des différentes Maisons. On s'est limité à mettre en évidence les différences les plus significatives, et de les illustrer par quelques exemples, sans prétendre à l'exhaustivité.

\section{Interdisciplinarité}

C'est là probablement le principe le plus souvent évoqué dans les rapports étudiés, ce qui confirme l'importance que lui accorde la doctrine du Réseau en l'énonçant en premier lieu. Les informations et réflexions qui le concernent peuvent être discutées en

\footnotetext{
65 - «Les structures fédératives : un essai de présentation », (2003), op. cit.

66 - (COLL.) Le réseau national des MSH (plaquette réalisée par la Maison des sciences de l'Homme d'Aquitaine, en collaboration avec les autres Maisons membres du Réseau), 2004 (disponible sur http : //www.msh-reseau.prd.fr/; existe également en version anglaise : National Network of social Sciences and Humanities Research Institutes).
} 
quatre points. Par définition, la première condition de l'interdisciplinarité est la coexistence de plusieurs disciplines, c'est-à-dire la pluridisciplinarité. Quant à cet aspect, il convient de commenter brièvement l'éventail des disciplines représentées dans les différentes Maisons, ainsi que dans le Réseau dans son ensemble. À la pluridisciplinarité s'ajoute ensuite une exigence d'échange et de coopération, voire de fusion entre les disciplines - visée que traduit plus spécifiquement le terme transdisciplinarité. II s'agira donc de rendre compte des modalités concrètes de mise en rapport entre disciplines. Au terme de cette esquisse d'inventaire, une question méritera d'être reprise : celle du statut, dans le travail interdisciplinaire, des différentes disciplines impliquées. Enfin, il faudra revenir à la question essentielle des finalités de l'interdisciplinarité.

\section{Eventail des disciplines}

On trouve dans les MSH toutes les principales disciplines des SHS, ainsi que nombre de disciplines plus pointues. À noter aussi la présence de disciplines qui débordent le domaine des SHS: sciences de la vie, de la terre, de l'environnement, etc. Ceci révèle l'ouverture disciplinaire du Réseau, selon l'acception la plus forte du principe d'interdisciplinarité : ouverture non seulement entre SHS, mais de celles-ci à l'égard des autres domaines scientifiques (convention, article 3 : « relations [...] entre les SHS et les autres secteurs scientifiques »). Cette ouverture s'opère d'ailleurs non seulement par l'inclusion d'équipes spécialisées dans ces disciplines, mais aussi par des collaborations plus ponctuelles, dans le cadre de certains projets. Pensons par exemple aux collaborations avec des ingénieurs, comme à Grenoble, ou avec des médecins (Nantes) ou les neurosciences (Lyon). 
Deux questions stratégiques que l'on pourrait poser ici - mais que nous laisserons ouvertes - sont celles-ci : quel est le poids souhaitable de l'ensemble des disciplines non SHS dans le Réseau ? Quelles sont, plus précisément, les disciplines qu'il serait particulièrement souhaitable de mieux impliquer?

Les orientations actuelles des MSH font émerger deux secteurs de fort intérêt : les sciences et techniques d'information et communication, la création artistique (par exemple, l'axe «industries de la culture et des arts » à Saint-Denis, ou le travail sur le « mouvement dansé », à Poitiers).

II faut reconnaître que certaines disciplines sont plus fréquemment représentées dans les MSH : I'histoire (16 mentions parmi les MSH françaises), la sociologie (14 mentions), la géographie et le droit (12 mentions), l'archéologie et les sciences du langage (11 mentions), la Littérature, les sciences économiques et la psychologie (10 mentions), la philosophie et l'anthropologie (9 mentions). Une position intermédiaire est occupée par les sciences et techniques de l'information et de la communication (7 mentions). II n'est pas aisé d'évaluer quelles disciplines devraient être renforcées en priorité 6 .

Un critère sociologique consisterait à se baser sur la liste des grands domaines différenciés d'activité sociale que, par ailleurs, la sociologie tend au long de ces dernières décennies à abandonner à des disciplines spécialisées : l'économie, la politique, le droit, 
l'art, la religion, l'éducation, etc. ${ }^{68}$ Sur la base de ce critère, on pourrait souhaiter une représentation un peu plus forte des sciences économiques et de la gestion d'entreprise. Le même critère conduit à constater la faible proportion de Maisons où sont abordés la science politique, mais aussi les domaines de l'art, de l'éducation, et de la religion. Enfin, par rapport à sa place dans l'université contemporaine, l'architecture peut être considérée comme nettement sous-représentée.

Quant aux éventails de disciplines des différentes Maisons, lesquelles comprenent entre une demi-douzaine et une quinzaine de disciplines, ils répondent tous à l'exigence d'interdisciplinarité posée par la charte. Par ailleurs, les développements annoncés vont dans le sens d'un élargissement de ces éventails.

Plus difficile est la caractérisation qualitative des configurations de disciplines. Trois Maisons présentent un profil similaire : la MOM, la Maison René-Ginouvès, et Nice, avec les constellations anthropologie-ethnologie-histoire-archéologie ${ }^{69}$.

D'autres configurations caractéristiques, en ceci que des groupes déterminés de disciplines y sont plus particulièrement représentés, sont, avec un groupe «focalisant », Strasbourg (histoire-archéologie) et Tours (géographie-urbanisme), ou, avec deux groupes, Aix (histoire-archéologie et géographie urbanisme), Caen (histoire-archéologie et sciences du langage-philologie-littérature) et Poitiers (sciences du langage-philologie-littérature-philosophie et

68 - La différenciation fonctionnelle à laquelle il est ici fait allusion a été discutée de manière particulièrement approfondie par Niklas Luhmann, qui s'est inspiré en particulier des travaux de Talcott Parsons. 
gestion-sciences économiques). Les autres Maisons apparaissent comme plus « généralistes » ${ }^{70}$.

Enfin, un travail qui reste à faire est une carte des constellations disciplinaires effectivement mises en œuvre dans la recherche. Une appréciation qu'il faudrait pouvoir fonder sur un inventaire plus méthodique est celle-ci : il semble bien que des réseaux de collaboration, par ailleurs denses et diversifiés, laissent pourtant subsister une certaine distance entre des travaux ayant intensivement recours à la coopération avec les sciences cognitives et les sciences et techniques de l'information et de la communication, d'une part, et d'autres, basés sur les méthodes plus traditionnelles de recueil et d'interprétation des données. Ce qui confirmerait le diagnostic de Jean-Pierre Dupuy quant à la réalité actuelle de l'écart entre les « deux cultures » en sciences sociales ${ }^{71}$.

\section{Transdisciplinarité}

Les rapports analysés présentent diverses modalités d'interdisciplinarité :

- projets associant des chercheurs de différentes disciplines ;

- équipes ou centres réunissant des chercheurs de différentes disciplines;

- filières de formation interdisciplinaires ;

- encadrement pluridisciplinaire de thèses de doctorat ${ }^{72}$;

70 - Ces profils devront également être discutés infra, en rapport avec le principe d'identification.

71 - Voir Re-inventing the Social Sciences, OCDE, (2004), p. 26 ss.

72 - Une modalité sur laquelle insiste le rapport Ouvrir les sciences sociales, Rapport de la Commission Gulbenkian pour la restructuration des sciences sociales (présidée par Immanuel Wallerstein), Paris, Descartes et Cie, 1996 (édition originale: Open the Social Sciences, Stanford University Press, 1996). 
- rencontres, séminaires, colloques, etc., destinés spécialement aux échanges de vue entre spécialistes de différentes disciplines sur des thèmes de recherche;

- échanges entre chercheurs favorisés par l'utilisation commune de certains équipements ou instruments ; - échanges entre chercheurs favorisés par le partage des mêmes locaux;

- représentation de différentes disciplines dans des organes de gestion, de consultation ou d'évaluation de la recherche.

Une dimension supplémentaire mérite encore d'être prise en compte: dans certaines initiatives, toutes les disciplines associées peuvent avoir un poids similaire; dans d'autres, au contraire, une discipline est clairement présentée comme « prépondérante » ou «de base ».

Ces diverses modalités correspondent évidemment à des degrés différents d'intensité des échanges. La question de savoir quelles modalités ou combinaisons de modalités méritent particulièrement d'être encouragées ne sera pas discutée dans le présent chapitre. Elle ne saurait, d'ailleurs, être traitée sans que soient d'abord explicitées les finalités de l'interdisciplinarité.

On se limitera ici à quelques mots concernant les différents moyens employés pour favoriser ces diverses modalités. La définition de projets interdisciplinaires, en particulier, peut être encouragée par des procédures spécifiques de consultation inter-équipes dans la perspective de la formulation des axes de recherche de la MSH (Toulouse). Une autre formule consiste à privilégier le critère de l'interdisciplinarité dans les appels d'offres internes (Caen, Réponse à la navette Ministérielle p. 5) 


\section{Statut des différentes disciplines}

Entre les diverses manières que les $\mathrm{MSH}$ ont de rendre compte du travail interdisciplinaire, une différence mérite d'être relevée: certaines présentations font état précisément des disciplines engagées; d'autres justifient l'interdisciplinarité de la recherche en soulignant le caractère fédérateur d'un thème ou d'un outil de travail (un exemple parmi beaucoup d'autres, à la MSH d'Aix : la promotion d'une " approche intégrée des cultures et des territoires »), sans mentionner les disciplines engagées. Par ailleurs, les listes des différentes personnes impliquées dans les projets (coordonnateurs, chercheurs, chercheurs invités, doctorants, etc.) peuvent, ou non, mentionner les disciplines d'appartenance.

Les laboratoires associés à un axe de recherche ou à un projet peuvent avoir un caractère disciplinaire ou être eux-mêmes déjà interdisciplinaires. La présentation d'un axe de recherche peut inclure, ou non, une mention explicite des disciplines associées, assortie éventuellement d'une justification des contributions des unes et des autres. Enfin, plus méthodiquement, ces présentations peuvent inclure un état de la recherche spécifique pour chaque discipline impliquée.

Au-delà de ces mentions des disciplines dans la documentation institutionnelle, la question qui mériterait d'être approfondie est celle du statut effectif des disciplines dans les différentes MSH. Telles qu'elles peuvent être reconstruites à partir des rapports analysés, leurs politiques sur ce point pourraient se distribuer entre deux pôles : d'un côté, une option «fusionnelle » (effacement des disciplines au profit de l'affirmation de thèmes intégrateurs), d'un autre une option «fédérative » (interdisciplinarité conçue comme 
l'intensification des rapports entre différentes disciplines, chacune clairement identifiée).

La documentation étudiée porte à adopter une attitude très ouverte face à cette alternative. Certes, l'affirmation d'une option fusionnelle peut correspondre à un degré élevé d'intensité et d'effectivité du travail interdisciplinaire. Mais un programme fédérateur peut, lui aussi, conduire à une production authentiquement interdisciplinaire, et garantir à celle-ci de bonnes conditions lorsque des unités à fort caractère disciplinaire jouent un rôle important dans la création et la vie d'une Maison. Il peut même favoriser l'identification et surtout le renouvellement des thèmes "fédérateurs", lesquels peuvent être plus facilement mis en débat, dès lors que l'identité de la Maison et son caractère interdisciplinaire découlent non seulement de ceux-ci mais aussi de l'engagement interdisciplinaire d'entités ou de chercheurs par ailleurs clairement situés en termes disciplinaires.

\section{Finalités et évaluation de l'interdisciplinarité}

D'autres l'ont déjà relevé : dans la réalisation du principe d'interdisciplinarité, il faut toujours être attentif au risque d'une « interdisciplinarité purement formelle $»^{73}$. La fréquence même avec laquelle apparaissent les termes «interdisciplinarité », «multidisciplinarité », « pluridisciplinarité », « transdisciplinarité », montre que ce principe a été perçu comme correspondant à une attente forte des instances fédératrices et de tutelle. Une telle perception peut évidemment, sans préjudice de la réalité des efforts, favoriser un usage symbolique de ces termes.

73 - Pour une politique des sciences de l'homme et de la société, (2001), op. cit., p. 195 ; Godelier Maurice, (2001), op. cit., p. 48. 
Il s'agit donc de bien évaluer l'effectivité de l'interdisciplinarité pratiquée. Une telle évaluation exige cependant que soient explicitées les finalités, et appelle une réflexion sur le degré optimal d'interdisciplinarité.

Les rapports étudiés soulignent plus particulièrement deux finalités. D'abord, l'interdisciplinarité favorise l'émergence de nouveaux thèmes, ou le renouvellement conceptuel. Cette idée est dans certains cas illustrée de manière particulièrement concrète et suggestive, comme c'est le cas pour les thèmes «Alimentations en Méditerranée » à Aix, ou encore " Concurrence », à Toulouse. On a cependant aussi pu relever que le voisinage entre les disciplines ne mène pas nécessairement à l'identification de thèmes effectivement intégrateurs, et mettre en garde face aux «programmes parapluies artificiels », comme ce fut le cas à Nanterre.

Une deuxième finalité est la stimulation du travail individuel des chercheurs, relevée dans plusieurs rapports.

Une troisième finalité pourrait être la stimulation des disciplines impliquées dans les entreprises interdisciplinaires, en particulier au plan méthodologique. II est naturel que cette finalité ne soit guère prise en compte par les rapports étudiés, élaborés dans le cadre d'institutions à vocation interdisciplinaire, et ne se destinant donc pas à considérer le développement scientifique dans la perspective des disciplines. Cependant, dans une évaluation globale de l'impact des MSH sur le développement des SHS, il vaudrait évidemment la peine de chercher à savoir comment des entités identifiées disciplinairement (départements universitaires, associations spécialisées, etc.) évaluent les bénéfices qu'elles retirent de l'existence de l'outil spécifique que représentent les MSH. Cette évaluation dépendra probablement non seulement de l'apport substantiel de celles-ci, 
mais aussi, dans une certaine mesure, des rapports institutionnels qui auront été établis entre les MSH et ces entités.

On a beaucoup, et justement, insisté, au long des trente dernières années, sur l'importance du travail interdisciplinaire, sur les problèmes graves que posent les cloisonnements entre disciplines, et sur la nécessité absolue, face à la plupart des thèmes correspondant à des préoccupations sociales ou à des priorités politiques, de composer les approches de plusieurs disciplines. À l'heure actuelle, il serait utile de faire aussi le bilan de ce que les disciplines universitaires ont apporté, en particulier en termes de formation des chercheurs, et de formulation de thèmes qui ont enrichi le débat social et politique justement parce qu'ils étaient apparus dans un autre contexte. Au risque de préjuger des conclusions d'une telle évaluation, disons que la science, de même que ceux qui lui adressent leurs demandes de connaissance, ont probablement beaucoup à gagner d'un équilibre et d'une bonne articulation entre démarches disciplinaire et interdisciplinaire, lesquelles méritent l'une et l'autre des moyens appropriés. Dans cet esprit, il reviendrait aux MSH et à leur Réseau à la fois de pratiquer l'interdisciplinarité de manière lisible et imaginative, et de s'assurer des ressources qui lui sont nécessaires, mais aussi de formuler une conception claire de leurs relations aux disciplines existantes. À considérer les rapports d'activité étudiés, tels que résumés ci-dessus, la pratique actuelle des MSH prend en compte ces deux aspects, le deuxième méritant pourtant d'être mieux explicité, dans le sens même d'un concept précis et équilibré d'interdisciplinarité.

\section{Dynamique interinstitutionnelle}

Des « trois i », c'est celui qui est le moins abondamment traité dans les rapports étudiés. 
Il est vrai, que les principes d'interdisciplinarité et d'internationalisation ont été incorporés il y a déjà longtemps dans la réflexion sur l'activité scientifique. L'aspect institutionnel, en revanche, a surgi plus récemment, après une longue période de développement pour ainsi dire linéaire du monde universitaire, durant laquelle les données institutionnelles pouvaient être considérées comme des constantes acquises.

Les rapports étudiés mettent en lumière la pluralité, caractéristique aujourd'hui de beaucoup d'organisations, des relations institutionnelles dans lesquelles s'insèrent les $\mathrm{MSH}$. On passera d'abord en revue l'éventail de ces relations, pour conclure par quelques réflexions sur la finalité de ces rapports.

Les entités avec lesquelles les MSH entretiennent des rapports institutionnels peuvent être réparties en au moins huit plans, les deux premiers devant être qualifiés de constitutifs :

- les entités de rattachement (une ou plusieurs universités; le CNRS);

- les unités fédérées, équipes ou centres de recherche ;

- le Réseau national des MSH et les autres MSH ;

- les autorités régionales, départementales et municipales ;

- des universités ou entités de recherche autres que les entités de rattachement, en particulier établies à l'étranger ;

- des associations et réseaux actifs dans les domaines de recherche de la MSH;

- des organisations internationales ;

- des partenaires régionaux.

Quant à la dynamique des rapports avec les entités de rattachement, on note des différences assez marquées entre les Maisons 
rattachées à une seule université et celles créées sur la base d'un partenariat entre plusieurs universités. La nécessité d’organiser ce partenariat semblerait favoriser de manière générale l'aptitude de la Maison à gérer les rapports interinstitutionnels. Au-delà de l'aspect administratif, I'option interinstitutionnelle peut se traduire dans l'organisation des différents axes de recherche de la MSH, comme par exemple à Saint-Denis, où chaque thème de recherche est placé sous la responsabilité de deux chercheurs, un de chacune des deux universités de rattachement.

Quant à la gestion des rapports entre unités fédérées, il serait intéressant de disposer de données plus détaillées sur la pratique dans le fonctionnement des Maisons (parmi les formules évoquées : le conseil des directeurs, dans la MSH de Paris, ou les réunions interéquipes, par exemple à Poitiers). Une question importante est celle de l'équilibre à trouver entre la place conférée aux unités fédérées dans l'organisation de la Maison et celle accordée aux instances d'orientation des axes de recherche propres des Maisons ${ }^{74}$ (exemple d'une organisation plaçant les axes et thèmes de la Maison au premier plan : Saint-Denis).

Les rapports étudiés mettent généralement en valeur les liens de coopération établis avec les autres Maisons. Le Réseau y figure également comme une composante importante de l'environnement institutionnel des MSH. Pour les Maisons créées il y a plus longtemps, la participation à la fondation du Réseau est mentionnée comme un moment important dans leur développement.

\footnotetext{
74 - Aurenche Olivier, (2003), «Maisons des sciences de l'Homme ou auberges espagnoles ? À propos de l'organisation interne des MSH », Sciences de l'homme et de la société. Lettre du département, n0 68, numéro spécial : Les structures fédératives (Paris, CNRS), août 2003, p. 19-21.
} 
Les Maisons établies récemment rappellent son intervention dans le processus de leur création. Sont fréquentes aussi les mentions des contrats d'objectifs soutenus par l'ACI «Réseau des MSH » comme source de financement de projets de recherche. Enfin, les rapports étudiés montrent comment certaines Maisons perçoivent un rôle spécial dans le cadre du Réseau, auquel elles apportent une contribution particulière (exemples: Grenoble:page web du Réseau ; Lyon ISH : réponse du Réseau à l'ACI numérisation).

Les autorités régionales, départementales et municipales sont mentionnées à propos des organes de gestion des Maisons. Leur rôle est particulièrement important dans la phase de création et dans la mise à disposition ou la construction des locaux. II serait intéressant d'approfondir l'analyse de leur rôle dans le fonctionnement ultérieur des Maisons.

Les rapports avec les institutions étrangères s'établissent généralement à l'occasion de coopérations de recherche et de visites de chercheurs. Ils concernent donc en première ligne des unités ou des responsables de recherche en particulier. Dans ces conditions, il revient à la MSH en tant que telle d'assurer un suivi des coopérations engagées et de proposer une vue d'ensemble sur le tissu de liens institutionnels qui se construit de la sorte (voir à ce propos la formule du « Cycle d'accompagnement de la recherche » mis sur pieds à Aix). Par ailleurs, si l'engagement personnel de certains chercheurs est la condition sine qua non de toute coopération académique interinstitutionnelle, il est certain que celui-ci sera stimulé par une reconnaissance appropriée au sein de leur institution d'appartenance. Cette reconnaissance institutionnelle est par ailleurs très utile, voire nécessaire, dans le traitement de problèmes administratifs qui se posent notamment dans les projets internationaux. 
Les associations et réseaux scientifiques (autres que les réseaux européens) sont relativement rares. Une modalité importante de structuration de tels rapports est l'action de certaines MSH dans le domaine de l'édition de revues. Étant donné l'importance des associations dans le développement des SHS, les rapports entre elles et les MSH mériteraient d'être développés. II est vrai que beaucoup d'associations et de réseaux ont une définition disciplinaire. On retrouve ici la question de l'équilibre nécessaire entre la promotion exigeante de l'interdisciplinarité et la reconnaissance appropriée des disciplines constituées. Une autre modalité d'établissement de rapports institutionnels avec des réseaux et associations est l'hébergement de sites web (exemples: site web de Lyon ISH, page « présentation »; site web de la MSH Paris).

Les partenaires régionaux seront abordés infra. On notera ici que l'établissement d'un réseau diversifié de partenaires institutionnels d'implantation locale peut être une garantie, à la fois d'une meilleure sensibilité aux intérêts et préoccupations locales, et d'une plus grande autonomie dans les rapports avec chacun de ces partenaires pris individuellement.

Pour conclure ce point, reconnaissons que le travail d'entretien d'un réseau complexe de relations interinstitutionnelles représente une charge de travail considérable. Et les moyens disponibles dans les Maisons pour cette activité seront toujours nécessairement limités. II est donc probable que, dans la pratique, certaines Maisons devront se focaliser sur certaines de ces relations, en se limitant pour le reste à une action plus modeste. Dans ces stratégies globales de gestion des rapports interinstitutionnels, il conviendrait d'accorder une attention particulière à l'équilibre entre le régional (entités de rattachement; autorités locales, départementales ou régionales; autres partenaires locaux) et 
l'international (institutions académiques, réseaux, associations, etc.).

L'importance de l'aspect interinstitutionnel dans l'action des MSH est étroitement liée à leur rôle dans l'articulation entre le CNRS et les universités dans la recherche en SHS. Dans la pratique, l'insertion des équipes accueillies et le renouvellement des thèmes de recherche conduit à formuler une finalité plus générale. On pourrait en effet soutenir le raisonnement suivant: diverses fonctions nécessaires à la recherche - en particulier la formation de base des chercheurs et l'aménagement de carrières professionnelles offrant une sécurité suffisante aux intéressés et une valorisation soutenue des compétences - exigent des organisations très solidement structurées et donc d'une malléabilité limitée (typiquement: les universités). Les caractéristiques de telles institutions peuvent entrer en conflit avec d'autres exigences de la recherche, en particulier celle de pouvoir réagir rapidement à de nouveaux problèmes ou demandes.

Une solution, face à ce dilemme des organisations «lourdes », est le développement, en articulation avec celles-ci, d'organisations plus légères, déchargées des fonctions très absorbantes mais nécessaires de «reproduction», et investies d'une responsabilité particulière de flexibilité thématique et donc organisationnelle. La complémentarité entre les deux types d'organisation exige cependant une concertation très poussée, et donc des relations stables et clairement définies. C'est à des relations de ce type que le principe d'« interinstitutionnalité » engage les MSH.

\section{Orientation internationale}

Tout comme l'interdisciplinarité, l'orientation internationale est un principe très fréquemment invoqué dans les rapports étudiés. Ici 
encore, il s'agit d'abord de rappeler selon quelles différentes modalités il est mis en œuvre. Les rapports fournissent ensuite matière à une brève réflexion sur les finalités de l'internationalisation. Enfin, au-delà des finalités principales, on rappellera quelques effets possibles, qui ne constitueraient pas à eux seuls des justifications, mais peuvent fournir des motifs supplémentaires de développer cette dimension.

\section{Modalités de l'internationalisation}

On recence les modalités suivantes:

- La conception et mise en œuvre de projets de recherche associant des laboratoires et chercheurs de différents pays. Dans cette modalité, les projets européens acquièrent une importance grandissante. Sur ce point, on peut encore distinguer les collaborations entre équipes et la participation, au sein de mêmes équipes, de chercheurs de différents pays ${ }^{75}$.

- Les collaborations internationales à long terme dans le cadre de revues (exemples: Caen : Revue internationale « Histoire et sociétés rurales »; Poitiers: Revue européenne des migrations internationales) ou de réseaux visant le développement d'autres outils de travail (coopération entre bibliothèques, connexion entre bases de données, coopération dans la conception et interconnexion de sites web, etc.; exemple : Nanterre).

- Les événements visant à mettre en contact des chercheurs de différents pays (colloques, séminaires, etc.). Ceux-ci peuvent être organisés par une $\mathrm{MSH}$; ou encore des chercheurs

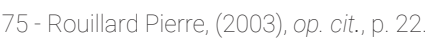


appartenant à des équipes associées à une MSH peuvent participer à des rencontres organisées à l'étranger (les mentions de tels événements abondent dans les rapports).

- La participation de collègues étrangers dans les instances de consultation scientifique ou d'évaluation, soit des Maisons, soit éventuellement de projets plus particuliers menés dans leur cadre (revues, collections de publication) $)^{76}$.

- Les filières de formation associant des chercheurs ou des institutions de différents pays (exemple: Clermont-Ferrand).

- Enfin, une modalité fréquemment mentionnée, et considérée comme caractéristique des MSH - en rapport avec leur nature de «Maison», lieu où l'on peut vivre - est l'accueil de chercheurs de l'étranger pour des séjours d'une certaine durée et pour participer aux activités de la Maison (ainsi à Nantes où est également mentionnée l'existence d'une association « chercheurs étrangers »).

Pour une appréciation plus détaillée des dynamiques d'internationalisation, il convient de tenir compte du poids respectif des initiatives des MSH et de celles des équipes ou centres qu'elles fédèrent. Les Maisons peuvent encourager les initiatives internationales par leurs appels d'offres internes ou dans le cadre des procédures de définition des axes de recherche. Elles peuvent les valoriser à travers des événements ou publications maison, ou les faciliter par l'accueil temporaire de chercheurs étrangers partenaires. La matière des échanges internationaux, en revanche, se construit beaucoup dans l'activité de 
recherche menée dans les centres et équipes. La valorisation parallèle de ces deux contributions est probablement un facteur de succès des politiques d'internationalisation : dans la documentation élaborée et divulguée par les Maisons, les mentions de participation à des initiatives internationales sont fréquentes. Une évaluation plus poussée pourrait examiner les modalités de promotion des MSH et du Réseau, dans le cadre de rencontres ou de publications à l'étranger, par les chercheurs qui y participent.

Une initiative qui articule d'une manière particulièrement intéressante plusieurs modalités d'internationalisation, en les combinant avec un travail d'intensification des relations interinstitutionnelles, est le cycle d'accompagnement à la recherche organisé à AixMarseille. Des personnalités étrangères sont associées à un programme de formation conçu pour fonctionner sur une durée de quatre ans, et dans le cadre duquel elles viennent passer un mois par année à la MSH pour accompagner sur place des recherches en cours. Ces séjours prolongés et périodiques garantissent l'établissement de relations fortes non seulement entre la personnalité invitée et les communautés des chercheurs travaillant à la MSH d'Aix-Marseille, mais également entre celle-ci et les institutions d'origine des invités. Et, dans ces conditions, les invités acquièrent en quelque sorte un statut de correspondants extérieurs de la $\mathrm{MSH}^{77}$.

\section{Finalités de l'internalisation}

Si l'importance de l'internationalisation n'est aujourd'hui plus mise en doute, il vaut pourtant la peine de rappeler quels bénéfices on peut 
espérer en retirer. Sur ce point, les rapports étudiés sont instructifs, par les trois situations assez différentes qu'ils mettent en lumière.

Certains thèmes exigent en eux-mêmes un travail international, en raison de leur propre caractère international. Un exemple classique, qui concerne plusieurs MSH, est celui des migrations. On pourrait également citer ici l'étude de moments historiques intéressant plusieurs pays : la Seconde Guerre mondiale (comme à Caen et à Strasbourg), ou encore certains aspects de la construction européenne (voir notamment les projets s'inscrivant dans des actions COST ou dans les programmes cadre de I'Union européenne, par exemple à Grenoble). Un exemple tiré d'un domaine très différent : la traduction.

Le cas de figure peut-être le plus courant est celui de projets engagés à une échelle internationale avec l'objectif de permettre une meilleure prise de recul par rapport aux réalités locales (comme à Caen, pour la recherche dans le domaine des études rurales). De tels projets pourront consister essentiellement en la comparaison des résultats de recherche. Ils peuvent aussi, ce qui suppose un effort plus considérable, impliquer la participation à la recherche, ici, de collègues étrangers, en même temps que, dans les recherches menées dans d'autres pays, la participation de chercheurs d'ici.

Enfin, un objet extérieur peut être traité en coopération avec des partenaires étrangers, sans pourtant avoir un caractère nécessairement comparatif impliquant une certaine réciprocité des opérations. Deux cas de ce type se présentent dans les rapports étudiés. L'un est l'étude d'objets présentant un intérêt universel, pour laquelle des compétences particulières existent en France. Pensons à des 
activités de fouilles archéologiques en différents points du globe (exemples : Lyon MOM, Nanterre). L'autre, assez différent, est celui d'opérations ayant un caractère plus marqué de coopération ou de solidarité internationale. Pensons au travail mené à Caen avec des collègues russes sur la mémoire de la catastrophe de Tchernobyl.

Ces trois situations présentent sans doute des similitudes et pourront, dans certains cas, se recouper. II n'en vaut pas moins la peine d'en faire une analyse distincte. En effet, de l'une à l'autre, les conditions de collaboration entre chercheurs impliqués, de négociation des moyens nécessaires, etc., peuvent varier significativement.

Les trois motifs qui viennent d'être exposés concernent l'internationalisation des SHS en général. S'agissant plus spécifiquement de l'activité des MSH et de leur Réseau, un motif de poids vient ajouter aux précédents. Les MSH peuvent, à l'échelle régionale, jouer un rôle non négligeable d'ouverture sur le monde global. Leur vocation internationale ne tient ainsi pas seulement à un engagement pris envers la communauté scientifique, mais également à un engagement envers leur lieu d'implantation ${ }^{78}$. Si les trois premiers motifs concernent avant tout l'orientation stratégique des laboratoires, centres et équipes, ce dernier concerne les instances de direction des Maisons, auxquelles il appartient de tirer parti de l'implication internationale des équipes au moment de produire, à l'adresse des collectivités locales et régionales, une offre bien conçue de visions sur l'international. Cet aspect renvoie cependant au thème de l'inscription locale et régionale, qui sera abordé ci-dessous.

78 - Sur les rapports entre l'internationalisation et l'inscription régionale, voir Commaille, Rouillard, (2004), op. cit., p. 8. 


\section{Motifs supplémentaires de développement à l'international}

Au-delà des raisons de fond qui justifient l'engagement d'une MSH ou des équipes qui la constituent dans des projets internationaux, on reconnaît à l'internationalisation d'autres effets bénéfiques.

Il est aujourd'hui acquis qu'un haut degré d'internationalisation est un facteur de prestige, qui jouera en faveur de l'institution dans les procédures d'évaluation. Plusieurs des rapports analysés soulignent tout particulièrement, parmi les effets bénéfiques du Réseau, le fait qu'il soit propre à favoriser l'internationalisation des Maisons membres. Ce qui est sans doute lié à la perception de cette plus-value que donne l'internationalisation.

La présence et la participation de chercheurs étrangers sont aussi perçues comme agissant de manière positive sur les relations entre les chercheurs locaux, par exemple : à Aix.

Enfin, une solide implication dans des réseaux internationaux est sans doute un bon antidote au «localisme ».

Si l'international est un bon antidote au «localisme», comme tout antidote, il peut devenir nocif à hautes doses. La disponibilité de tout chercheur étant limitée, le temps consacré à la correspondance et aux voyages requis pour la réalisation de projets internationaux est forcément à déduire du temps qu'il peut consacrer aux échanges avec ses collègues locaux. Les rapports de proximité, sans être jamais suffisants, sont pourtant utiles, voire nécessaires (maintien d'une certaine culture d'interaction, de débat en face-àface ; possibilité de faire une analyse ou une évaluation partagée de l'institution pour laquelle on mène son travail de chercheur, des nécessaires articulations entre réseaux et entre sphères d'appartenance, etc.). II paraît ainsi souhaitable d'éviter tout processus 
d'érosion des rapports entre les chercheurs plus fortement impliqués dans l'international et ceux plus actifs sur place. De ce point de vue, le caractère de «maison » des MSH, avec une forte valorisation des espaces et des temps de rencontre, autant académiques que conviviaux, peut jouer un rôle très favorable.

Les principes d'orientation internationale et d'inscription locale et régionale doivent être donc être pensés de manière articulée.

\section{Inscription politique et sociale/ implantation locale et régionale}

Si ce principe n'est guère explicitement cité dans les rapports, de nombreuses mentions révèlent qu'il correspond bien à une préoccupation des responsables des $\mathrm{MSH}$. Ces mentions sont, cependant, de natures très variées, ce qui tient, d'abord, à la diversité des composantes du contexte local et régional. Dans les rapports avec ce contexte, diverses actions des MSH sont envisageables. Ici encore, l'analyse des discours et pratiques doit être l'occasion d'une réflexion sur les finalités propres à fonder le principe en cause. Enfin, il conviendra de rappeler quelques limites à prendre en compte dans sa mise en œuvre.

\section{Diversité des composantes du contexte local et régional}

L'éventail des entités constituant l'environnement local et régional des $\mathrm{MSH}$, inclut les composantes suivantes

- en première ligne, les entités de rattachement, avec lesquelles, cependant, se tissent principalement des rapports spéciaux de gestion et de coopération académique, déjà abordés supra au titre de rapports interinstitutionnels. Mais il peut aussi être question de rapports plus quotidiens au niveau de la vie de campus, lesquels méritent d'être considérés ici ; 
- les autorités municipales, départementales et régionales;

- des associations et entités analogues ;

- des entreprises ;

- la presse locale;

- certains rapports signalent des entités plus spécialisées avec lesquelles les MSH développent une coopération renforcée (pôle scientifique et technologique régional à Caen, pôle d'initiative régionale à Tours).

En regroupant ces diverses entités dans la catégorie de contexte local et régional, il s'agit de les considérer, non seulement en tant qu'institutions, mais en tant que possibles médiateurs d'intérêts, aspirations ou éventuellement préoccupations de la population. Au-delà de ces possibles «représentants ", c'est du tissu social environnant qu'il s'agit.

\section{Les pratiques}

On rencontre, au sein des MSH, des modalités très diverses de développement des rapports avec l'environnement local et régional. Une première distinction mérite d'être faite entre la prise en compte des intérêts locaux dans la définition des thèmes de recherche, d'un côté, et, d'un autre, l'adoption de mesures destinées à favoriser en général l'interaction avec le monde environnant, indépendamment de la définition des thèmes.

Quant aux thèmes à pertinence locale, deux grandes catégories se dessinent. D'une part, certains axes de recherche répondent clairement, et de manière assez similaire d'une MSH à l'autre, à une demande que l'on pourrait qualifier d'identification.

II s'agit de mieux connaître, de mieux penser, et de contribuer à la préservation de ce qui caractérise la région. Pensons aux travaux 
sur le «paysage»(Besançon, En direct), l'image des volcans (Clermont-Ferrand), les peintures rupestres (Toulouse), les archives d'entreprise (Dijon), etc.

D'autre part, certains thèmes correspondent à des problèmes de société présentant une certaine urgence ou nouveauté : les jeunes et la ville (Nantes), la santé (Saint Denis), les usages d'internet par le grand public (Poitiers), l'emploi, la violence domestique (Toulouse).

La distinction entre ces deux catégories de thèmes se justifie pour diverses raisons. En particulier, leurs conditions d'émergence diffèrent. Les premiers auront souvent une certaine tradition ; la MSH pourra ainsi parfois prolonger un travail déjà engagé par des musées, des sociétés savantes ou des personnalités locales. Les seconds auront surgi plus récemment et de manière plus diffuse. Par ailleurs, les premiers sont parfois très marqués par des données locales et, de ce fait, assez étroitement spécifiés, ce qui peut rendre difficiles des comparaisons au-delà de la région et suggérer qu'une telle comparaison n'est pas prioritaire. On note cependant de remarquables efforts dans le sens de l'internationalisation de ces thèmes de pertinence locale. Les seconds pourront correspondre à des problèmes intéressant des espaces plus vastes, mais c'est alors leur relative nouveauté qui peut faire que les partenaires extérieurs potentiels seront rares.

À propos de l'articulation entre les intérêts locaux et la recherche des $\mathrm{MSH}$, on signalera en particulier les entités qui en sont spécialement chargées, comme l'« office universitaire d'études normandes", le "GIS risques collectifs et situations de crise » ou encore le « service partenariat », à Nanterre. Pour la France en général, on signalera également l'Association nationale pour la valorisation de la recherche en SHS auprès des entreprises (ANVIE), qu'accueille la MSH Paris. 
Quant aux modalités d'intensification des interactions avec l'environnement, relevons les suivantes:

- des partenaires extérieurs (associations, entreprises) peuvent être, de diverses manières, associés à la réalisation de projets de recherche ou à la discussion de leurs résultats (exemples : Bordeaux, Poitiers) ;

- des recherches peuvent impliquer d'importantes phases de questionnement des populations et acteurs locaux (Nantes : usagers du système bancaire; juges) ;

- les MSH peuvent accepter de mener des recherches, ou d'élaborer des rapports d'expertise, pour le compte de partenaires extérieurs, au titre de service comme à Aix ou à Saint Denis ;

- elles peuvent mettre des locaux à disposition d'entités locales ;

- certaines initiatives des MSH peuvent être ouvertes au grand public : conférences, expositions, journées de la science ou Fête des SHS (exemple:Besançon, page web), journées «portes ouvertes », petits déjeuners d'information;

- des publications peuvent être conçues en vue d'une divulgation grand public (Grenoble : Lettre d'information sur la recherche en $L S H S)$;

- les bibliothèques semblent être, dans certaines Maisons, accessibles au grand public ;

- les MSH peuvent s'engager dans certaines manifestations extra muros ;

- des relations régulières peuvent être cultivées avec les médias locaux, en vue d'une meilleure couverture des activités de la Maison ;

- les rapports avec l'environnement peuvent être pris en compte dans la composition des instances d'accompagnement ; 
- un dispositif qui, en première ligne, a une fonction institutionnelle, mais qui mérite d'être rappelé ici, est la représentation des autorités régionales, départementales ou locales dans les instances de gestion des Maisons. II serait intéressant de mieux connaître l'apport de celle-ci dans la pratique, notamment dans l'effort de mise en rapport de l'activité scientifique avec la vie de la cité ;

- bien entendu, un dispositif important sont les documents de promotion institutionnelle et, surtout, les sites web (explicite dans ce sens, le site web de la MSH Claude Nicolas Ledoux à Besançon). II est vrai que cette information se destine à un public autre - la communauté scientifique internationale - que l'environnement de l'institution. Mais elle peut être conçue aussi pour aller au-devant des intérêts du public local, notamment par des éléments concernant la localisation concrète, et par la mise en valeur des missions d'intérêt général et des manifestations grand public.

L'image de la MSH au niveau local et régional, susceptible de favoriser l'effet des dispositifs qui viennent d'être mentionnés, dépend naturellement aussi de sa localisation et de son architecture, ainsi que, dans une mesure marginale, de tous les dispositifs de signalisation favorisant son repérage (fléchage, mention sur les plans officiels de la ville, etc.). La localisation et l'architecture sont abordées dans plusieurs rapports; la signalisation mériterait d'être aussi prise en compte dans des travaux d'évaluation à venir, même s'il s'agit d'un point secondaire.

Quant aux structures organisationnelles destinées à favoriser les interactions avec l'environnement, on note des différences assez marquées entre les Maisons. Dans certaines, les missions de 
communication avec l'environnement sont confiées à une unité particulière (comme à Grenoble, et Lyon MOM). Dans d'autres, elles se distribuent entre différents secteurs. Une participation active de plusieurs secteurs paraît une condition nécessaire à l'établissement de rapports effectifs avec l'environnement. Pourtant, l'existence d'une unité spécialisée, pour laquelle cet aspect constitue une priorité, est susceptible de favoriser la cohérence de l'image externe de l'institution dans son ensemble, et semble jouer en faveur d'une bonne inscription dans le contexte social et politique.

\section{Finalités}

La bonne acceptation d'une institution auprès de la population et des autorités de son lieu d'implantation est un facteur important de son bon fonctionnement et développement. D'un point de vue plus général, point de vue qu'il revient en particulier aux instances du Réseau de bien prendre en compte, l'inscription des MSH dans leur contexte local doit aussi être pensée dans le cadre de la question plus générale des rapports entre SHS et société.

Après les débats qui ont opposé naguère certaines sensibilités technocratiques et d'autres sensibilités plus critiques, on peut aujourd'hui admettre un consensus quant à la nécessité de favoriser des formes de participation sociale dans la construction des thèmes traités par la recherche en SHS et dans le débat sur les possibles usages de ses résultats ${ }^{79}$. Ce qui paraissait indispensable en raison de la nature même de l'objet des SHS est d'ailleurs, entre-temps, devenu aussi une priorité pour l'activité scientifique en général (voir l'importance croissante que prend actuellement,

79 - Particulièrement clair sur ce point: Hans van Ginkel, in : Re-inventing the Social Sciences, (2004), p. 8, avec ce titre programmatique: " Social Sciences in society : a new partnership »; et Luk van Langenhove, ibidem, p. 65. 
dans les sciences "dures», le thème "science et société »). Certes, cette participation s'effectue aussi par le biais de politiques publiques d'incitation et d'accompagnement de la recherche. Mais, face à la distance qui peut s'établir entre la sphère politique et les autres sphères sociales, et aux contraintes diverses que peut signifier l'agenda politique, il importe de favoriser d'autres modalités, moins formalisées et peut-être plus directes, d'échanges entre la collectivité et la recherche. L'échelle locale et régionale se prête particulièrement bien à des mesures de cet ordre. Les MSH, en raison de leur caractère de "maison », donc de leur forte visibilité locale, et d'une vocation de recherche en matière sociétale plus immédiatement perceptible de l'extérieur que dans le cas des universités, peuvent, dans ce domaine, jouer un rôle déterminant.

L'étude de l'information générale ${ }^{80}$ fournie par les médias au grand public en matière scientifique montre l'importance considérable qu'ont prise ces dernières années les sciences «dures », au premier rang desquelles la biologie, dans la réflexion sur les phénomènes humains. Plus probablement que naguère, le problème des SHS, avant toute question de participation, est d'abord celui de leur visibilité publique. Celle-ci a beaucoup à gagner d'une politique de présence locale et régionale des $\mathrm{MSH}$. Et cette politique sera plus efficace si elle s'inscrit dans un effort concerté du Réseau.

\section{Limites}

La bonne mise en œuvre du principe d'implantation locale et régionale exige que soient bien prises en compte certaines limites. La

80 - Considération basée sur un dossier de presse constitué aux fins d’appui à la recherche et à l'enseignement, réunissant depuis plusieurs années des documents tirés de journaux français, allemands, anglais, espagnols, portugais et suisses. II serait évidemment utile sur ce point de disposer de données réunies et analysées de manière plus systématique. 
première est rappelée avec insistance dans les travaux de réflexion sur le Réseau discutés ci-dessus : il s'agit du risque de «localisme». Le risque de «localisme » diminue considérablement dans la mesure où une Maison mène aussi de nombreuses activités dans des réseaux et sur des thèmes internationaux. À côté de l'équilibre entre l'aspect local et régional et l'aspect international des activités d'une Maison, il faut évidemment aussi être attentif aux modalités d'articulation effective entre eux. Cette articulation pourra être grandement favorisée par une politique clairement énoncée au niveau des directions des Maisons.

Un autre problème est celui du maintien de la nécessaire indépendance de la recherche. Très justement, le rapport de la MSH d'Aix insiste, à propos de la politique d'acceptation de mandats d'expertise, sur la nécessité de garantir, autant que possible, un accès public aux résultats obtenus dans le cadre de tels mandats. II est naturel que ce problème soit perçu avec une particulière acuité au niveau local. II faut cependant relever qu'il se pose aussi à l'échelle européenne ${ }^{81}$. En cette matière, une instance comme le Réseau serait bien placée pour agir dans le sens, en particulier, d'une meilleure garantie de la publicité des résultats de travaux d'expertise, de manière d'autant mieux fondée qu'il peut s'appuyer sur les expériences concrètes des Maisons en la matière. Un outil précieux dans l'approche de ces problèmes est l'observatoire de l'expertise créé à Lyon.

81 - Dans certains domaines en tout cas, les contrats d'expertise passés dans le cadre de projets menés sous l'égide de la Commission européenne incluent des clauses très strictes attribuant la propriété des résultats du travail à la Commission et imposant la confidentialité à l'expert. 
Au niveau régional, l'indépendance de la recherche pourra également être favorisée par une diversification des partenaires (associations, entreprises, établissements publics) visant une représentation de nombreux secteurs de ce que l'on pourrait appeler la société civile régionale.

Sur la question de l'indépendance de la recherche, il conviendrait de resituer les MSH dans le contexte plus vaste des dispositifs de production scientifique en SHS. Dans son ensemble, ce dispositif devrait avoir une grande autonomie, condition de la qualité du travail scientifique et de la plus-value que celui-ci doit apporter aux connaissances élaborées dans la pratique ${ }^{22}$. Cette autonomie n'est défendable que s'il existe des dispositifs efficaces d'articulation avec l'environnement social. Les MSH figurent parmi ces dispositifs. Dans l'orientation de leurs activités, l'insertion dans le tissu social, et donc aussi l'activité de service apportée aux entreprises, doit donc être une priorité. Les effets éventuellement contraignants d'une telle insertion devraient être compensés par les liens que les MSH doivent cultiver avec les autres organisations impliquées dans la production scientifique. Ceci renvoie au thème de l'insertion des MSH dans les réseaux scientifiques internationaux,

82 - Un parallèle mériterait d'être osé : face à la demande insistante d'une plus grande responsabilité sociale des entreprises, l'Economist (The good company. A survey of corporate social responsibility, 22 janvier 2005) défendait récemment avec brio la thèse provocatrice selon laquelle les entreprises servent le mieux la société en se limitant à assumer leur responsabilité économique de rentabilité. Pourquoi ne pas récuser la «responsabilité sociale » de la science avec l'argument selon lequel la société a surtout besoin d'une science qui produise une connaissance solide plutôt que d'une science inquiète de la demande sociale? Le parallèle a cependant des limites (qui n'ôtent rien à l'intérêt qu'il y aurait à l'approfondir) : des entreprises rentables gagnent, par définition, de l'argent, première et évidente condition de la poursuite de leur activité ; la valeur des connaissances que produit la science, nécessaires pourtant autant à la poursuite de l'activité scientifique elle-même qu'aux autres secteurs sociaux, est moins aisée à mesurer. 
dans les réseaux disciplinaires et dans un tissu de rapports institutionnels avec d'autres institutions à mission scientifique.

\section{Identité scientifique}

La charte prévoit que les MSH développent «des activités de recherche propres qui contribuent à définir leur identité scientifique » (article 2). Qu'en est-il, à l'heure actuelle, de cette « identité scientifique»?

Les MSH se constituent entre un plan d'entités de rattachement et un plan d'unités fédérées. Une première question est donc celle de l'identité propre de la MSH au sein de ce complexe institutionnel. Cette identité devra prendre appui, en première ligne, sur une activité de recherche et de formation doctorale qui marque clairement son rôle face aux entités de rattachement, et sur des axes de recherche assez clairement formulés par la MSH elle-même pour la distinguer des recherches menées par les unités fédérées. Mais il faut reconnaître que des facteurs institutionnels peuvent favoriser ou au contraire inhiber l'affirmation de cette identité. Celle-ci doit donc être une dimension à prendre en compte aussi dans la politique des rapports interinstitutionnels.

\section{Modalité d'affırmation}

La manière la plus simple d'affirmer son identité scientifique consiste à l'énoncer dans le propre nom de l'institution. Cette modalité est adoptée par quatre Maisons : la Maison de l'Orient et

\footnotetext{
83 - L'analyse, dans la première partie de cette note, de la doctrine du Réseau, qui privilégie les «trois i », conduit à traiter ce point en dernier lieu. Cette construction peut étonner, dans la mesure où la question de l'identité d'une institution pourrait être considérée comme préalable à toute autre. Le plan auquel nous avons été conduits est pourtant plus approprié : l'identité d'une institution ne se discute pas dans l'abstrait mais sur la base de travaux déjà engagés, auxquels elle confère de meilleures conditions de réalisation et de lisibilité.
} 
de la Méditerranée «Jean Pouillon » à Lyon, la Maison «RenéGinouvès» archéologie et ethnologie à Nanterre, la Maison des sciences de l'Homme "Villes et territoires » à Tours et, plus récemment, la Maison des sciences de l'Homme de Dijon - «Patrimoine, espace, mutations ». Ce qui a pu faire dire (avant Dijon) que « trois (MSH) seulement répondent au critère de la charte en affichant "la définition d'une identité scientifique [...]" " 84. L'affirmation de l'identité scientifique par le nom de l'institution n'est cependant pas la seule formule. Les textes de présentation des Maisons peuvent également être un véhicule approprié, pour autant que, dans ceux-ci, l'énoncé de la vocation de la Maison soit consistant et bien placé. Parmi ces textes, on peut penser notamment à la plaquette de présentation du Réseau ${ }^{85}$, la page d'accueil du site web de l'institution, ainsi que tout autre document de promotion institutionnelle (feuillet, prospectus, brochure). Cinq Maisons ont adopté cette modalité: Aix («Monde méditerranéen », l'adjectif « méditerranéen » figurant déjà, il est vrai, dans le nom), Dijon (« Patrimoines, espaces, mutations »), Nantes (« le lien social »), Nice («Frontières, marges, discontinuités »), Strasbourg («Études européennes/sciences de l’Antiquité »).

Les termes mêmes de la Charte- "activités de recherche propres qui contribuent à définir leur identité scientifique »- suggèrent une troisième modalité : l'identité peut surgir de la composition des domaines de recherche annoncés. Les éventails d'«axes de recherche » actuellement annoncés par les Maisons soulèvent de ce point de vue quatre problèmes. Premièrement, lorsque les axes sont nombreux (on en compte jusqu'à sept chez

84 - Aurenche Olivier, (2003), op. cit., p. 19

85 - Le réseau national des MSH, (2004), op. cit. 
les Maisons qui n'indiquent pas de thème fédérateur), l'hétérogénéité des thèmes peut compromettre l'émergence d'une identité suffisamment focalisée. Deuxièmement, certains thèmes sont traités dans de nombreuses Maisons. Ceux-ci se prêtent donc mal à l'identification d'une Maison parmi les autres qui constituent le Réseau. Troisièmement, les « axes de recherche » sont souvent redéfinis ${ }^{86}$, ceci conformément à l'exigence de renouvellement posée elle aussi par la charte. On peut se demander si une identité institutionnelle peut prendre appui directement sur des données aussi changeantes. Quatrièmement, enfin,-un point plus formel - la définition des axes de recherche contribue d'autant plus efficacement à l'identification de la Maison que ces axes sont eux-mêmes bien mis en valeur. À ce propos, l'observation d'Aurenche ${ }^{87}$ reste valable : les axes n'apparaissent pas toujours au premier plan de la présentation

Des problèmes qui viennent d'être évoqués, le plus délicat est celui que pose le renouvellement des thématiques. En effet, si les MSH doivent renouveler avec une certaine fréquence leurs recherches propres, tandis que, par ailleurs, elles doivent aussi se doter d'une « identité », c'est-à-dire un profil aisément identifiable et permettant une reconnaissance dans la durée, cela exige de leur part un effort permanent de reformulation de l'argumentaire justifiant ce profil, voire la capacité de redéfinir ce profil lui-même, si la nature des recherches mises en œuvre révèle une évolution plus marquée. Les rapports périodiques d'activités produits par les Maisons offrent un bon cadre pour une réflexion de cet ordre. Dans ce sens,

86 - Comparer, dans les documents de présentation des plans quadriennaux, les rapports sur la période en voie de conclusion et les perspectives pour la nouvelle période quadriennale. 
il serait souhaitable qu'ils incluent plus systématiquement un point consacré explicitement aux rapports entre les activités réalisées ou planifiées et l'identité globale de la Maison.

L'identité scientifique de la Maison, telle qu'elle résulte de son engagement dans un domaine ou dans des domaines spécifiques pourra être mise en évidence par la Maison à travers des manifestations servant sa visibilité, en particulier des publications (voir par exemple les Cahiers de la MRSH Caen, ou encore la récente publication de Tisser le lien social, par la MSH de Nantes), ou des « événements maison » (colloques annuels).

Notons ici que la question de l'identité scientifique est discutée de manière détaillée dans le cadre des procédures de création de nouvelles Maisons, ce qui révèle une bonne perception de ce principe.

Le Réseau lui-même pourrait éventuellement contribuer de manière plus ciblée à la divulgation du profil des différentes Maisons, bien entendu toujours sur la base des formulations fournies par celles-ci. Ainsi, la plaquette de présentation du Réseau pourrait, parmi les points d'information générale (date de création, statut, direction), inclure un point «dominantes». Par ailleurs, la page de présentation de l'ensemble des $\mathrm{MSH}^{88}$, sur le portail web du Réseau, pourrait inclure elle aussi, outre les noms et adresses des Maisons, un bref énoncé de leur vocation principale.

\section{Les thèmes}

Quant au contenu de l'identité énoncée, relevons d'abord que, en accord avec la Charte, qui parle d' «une ou plusieurs dominantes », certaines Maisons ont opté clairement pour une identité basée sur

88 - http : //www.msh-reseau.prd.fr/LesMSH/ 
deux ou trois thèmes différents. Ce peut être une excellente formule pour bien rendre compte de la diversité de l'offre et/ou de la demande régionale de recherche (explicites dans ce sens : Lyon, ISH, Grenoble, in: Les structures fédératives..., 2003, p. 33). Cependant, il pourrait être utile, pour une mise en œuvre cohérente du principe d'identité scientifique des Maisons, que le Réseau donne quelques orientations propres à éviter que la multiplication des thèmes conduise à une dilution du profil. Le cas de la Fondation MSH de Paris, dont la vocation, dans le cadre même du Réseau, est généraliste, fait sur ce point évidemment exception.

Les Maisons s'identifient généralement par référence à un ou plusieurs thèmes de recherche. Mais on trouve aussi la référence à des disciplines (Nanterre : archéologie/ethnologie) ${ }^{89}$. Dans les présentations faites de certaines autres Maisons, l'accent est placé parfois aussi sur les outils (ISH Lyon: le pôle image animée; voir aussi Aix : pôle de compétence image/son; Besançon : plate-forme technologique), sur des «objets fédérateurs » (Besançon : saline royale d'Arc et Senans; la forêt) ou encore sur un objet emblématique (Caen : le plan de Rome). Tout en reconnaissant le potentiel mobilisateur de tels objets identifiants, il faut cependant aussi reconnaître qu'ils peuvent favoriser une focalisation « localiste ».

Les thèmes énoncés, soit explicitement au titre de thème fédérateur, soit parmi les thèmes d'axes de recherche ${ }^{90}$, peuvent être

89 - Voir sur cette question l'essai de typologie des Maisons selon les disciplines représentées, supra.

90 - Linventaire qui suit ne tient pas compte de la globalité des projets menés dans les différentes Maisons. II s'agit uniquement de proposer une lecture du panorama que proposent les Maisons dans leur ensemble, par l'affirmation d'un grand thème ou la mise en évidence de leurs axes de recherche. 
distribués entre deux pôles : mentions uniques ou rares, d'un côté ; mentions fréquentes d'un autre.

Parmi les mentions uniques ou rares, et donc propres à conférer à une Maison une identité nettement marquée, on notera en particulier ceux qui se laissent aisément rattacher à une caractéristique de la ville ou région d'implantation : la mémoire à Caen, le risque à Grenoble ${ }^{91}$, les frontières à Nice, l'Europe à Strasbourg, l'entreprise à Toulouse. D'autres attirent l'attention par leur originalité. Par exemple l'innovation ou les espaces funéraires à Bordeaux, ou l'expertise à I'ISH (Lyon).

Dans cette même catégorie générale des mentions uniques ou rares, on peut placer également les thèmes à définition géographique: le monde méditerranéen (Aix, MOM Lyon, Montpellier), l'Afrique (Bordeaux), les Alpes (Grenoble, Nice), les mondes germaniques (Strasbourg). Proche de cette catégorie: les espaces francophones (Bordeaux). On peut ici se demander s'il ne serait pas envisageable que le Réseau, à long terme, stimule l'émergence et la valorisation de thèmes qui compléteraient cette prise en compte des «mondes voisins » : monde ibérique, monde anglosaxon², Benelux et Europe du Nord.

Parmi les mentions fréquentes, certaines peuvent être considérées comme révélant l'importance croissante de certaines problématiques, ou approches, bien au-delà des contextes d'implantation des Maisons. Problématiques : la santé (Lille, Nantes, Saint-Denis) ou migrations et multiculturalisme (Besançon, Nice, Poitiers).

91 - Au niveau des recherches, le risque est également traité à Aix, Caen et Lyon.

92 - La MSH de Rennes, en cours de création, envisage un axe de recherche « monde atlantique » 
Approche : les sciences cognitives (Caen, Clermont-Ferrand, Lille, Poitiers, Toulouse).

Enfin, on note la fréquence de mentions très directement liées à une demande régionale d'étude et de réflexion sur les données locales: les deux thèmes les plus fréquents sont le territoire et le patrimoine.

Le fait que plusieurs Maisons traitent un thème analogue contribue à marquer le profil du Réseau dans son ensemble. Dans ce sens, les deux types de mentions qui viennent d'être signalées suggèrent l'idée d'un réseau à la fois bien ancré régionalement et ouvert aux problématiques émergentes. Ce sont là des caractéristiques certainement positives, et qui correspondent aux principes à la fois d'inscription dans la cité et de renouvellement. Si le Réseau bénéficie ainsi de telles convergences pour son image d'ensemble, celles-ci entraînent cependant aussi une responsabilité. Dans ces domaines « de convergence », il est particulièrement important que le Réseau favorise les échanges et les initiatives communes (à l'instar de ce qui se fait en matière d'archéologie, à Nanterre). À la fois pour mieux tirer parti du potentiel de recherche et contribuer à une limitation des éventuels dédoublements, et pour mieux valoriser ces initiatives, au bénéfice autant des Maisons que du Réseau.

Une conclusion possible de l'inventaire qui précède est que, du moins dans bien des cas, une définition optimale de l'identité scientifique d'une Maison pourrait inclure à la fois un volet répondant plus directement à la demande régionale de meilleure connaissance de soi, et un volet lié à un thème plus original et pointu, peut-être émergeant à l'échelle internationale. Un cas de figure idéal est peut-être celui d'une problématique à la fois 
étroitement liée au lieu et mais dont l'intérêt transcende le lieu (on peut citer les exemples de la mémoire à Caen et des frontières à Nice). Mais une telle problématique ne pourra pas se trouver en tout lieu, et il ne serait pas souhaitable qu'elle s'impose trop massivement et définitivement à toutes les activités d'une institution.

\section{Remarques finales}

Au terme de la discussion séparée des cinq « i », à la lumière à la fois de la doctrine du réseau et de la pratique actuelle des MSH, relevons d'abord brièvement le fait que les cinq principes ainsi mis en évidence, comme on a pu le signaler à diverses reprises, «font système ». II existe en effet une remarquable complémentarité entre eux.

Certains se renforcent mutuellement. Ainsi, l'interdisciplinarité a tout à gagner d'une organisation bien pensée des rapports interinstitutionnels. Ou encore : I'inscription sociale et politique engage les MSH à donner de la substance aux rapports qu'elles cultivent avec leur environnement, tandis que le principe d'«interinstitutionnalité » met l'accent sur la lisibilité, la continuité et la nécessaire dimension organisationnelle de ces rapports.

D’autres s'équilibrent. L'orientation internationale peut préserver les MSH du risque de «localisme», alors qu'inversement l'inscription locale peut éviter l'érosion des interactions pratiques entre chercheurs, et entre les chercheurs et leur contexte d'action immédiat. L'orientation internationale et le développement des rapports interinstitutionnels peuvent contribuer à mieux définir la position des disciplines dans les MSH, en même temps que l'impératif d'interdisciplinarité interdit le rétablissement des cloisonnements existant ailleurs. 
D'autres, enfin, se « précisent » mutuellement. La réflexion sur les modalités d'implantation régionale et de culture des rapports interinstitutionnels conduit à un concept d'« identité institutionnelle», par rapport auquel le concept d'«identité scientifique » doit être investi d'un sens spécifique, lié plus étroitement au travail de production intellectuelle.

Notons ensuite que le système de ces cinq principes, s'il définit assez précisément l'orientation générale des activités des MSH, garantissant ainsi la cohérence d'ensemble de ces activités, laisse entièrement ouverts les choix thématiques, méthodologiques et organisationnels. En ces matières, en bonne politique scientifique, il convenait de laisser la plus grande autonomie possible aux MSH. Cette autonomie a cependant pour contrepartie une exigence à laquelle renvoie de manière très suggestive la notion de « gouvernance scientifique $»^{93}$. Notion propre à nous ramener, au terme d'une réflexion systématique sur les principes, à la question de l'évaluation, approfondie dans le chapitre qui suit. 
Avenir de la recherche 


\section{4 \\ Enjeux de l'évaluation des Maisons des sciences de l'Homme}

Birgit Arve-Parès

Linda Hantrais

es structures fédératives constituent en France un cas très particulier en matière d'évaluation. Le Réseau des Maisons des sciences de l'homme (MSH) est d'autant plus intéressant comme cas de figure qu'il s'agit d'une structure interinstitutionnelle et pluridisciplinaire au niveau national, et que chaque Maison est elle-même une structure de ce type. De par la diversité des disciplines et l'importance des effectifs de chercheurs et d'enseignants-chercheurs (environ 4 000) accueillis dans les Maisons, le Réseau occupe une place prépondérante dans le paysage de la recherche en sciences humaines et sociales. Cependant, la majorité des Maisons, en tant qu'unités mixtes de services (UMS), n'ont pas, paradoxalement, à être évaluées pour leur contribution scientifique en tant que structure de recherche. En

94 - Avec la participation de Jacques Commaille, Jacques Fontanille et Martine Bentaboulet 
outre, les dispositifs d'évaluation mis en place ne sont pas adaptés pour prendre en compte la pluridisciplinarité.

Ces particularités constituent pour le Réseau des MSH à la fois un problème ${ }^{95}$ et un défi, car il s'agit de trouver un mode d'évaluation qui soit d'une part assez souple pour ne pas surcharger des chercheurs et enseignants-chercheurs déjà soumis à de multiples évaluations, d'autre part assez rigoureux pour légitimer et crédibiliser le processus, dans un contexte où les SHS s'efforcent d'obtenir leurs lettres de noblesse.

Cette tâche est d'autant plus délicate que le concept d'évaluation tend à être utilisé en France de façon non différenciée pour désigner à la fois une appréciation ex ante de la qualité des prestations proposées, et un bilan a posteriori de la qualité des performances accomplies. Cet usage pose problème, du fait que, en principe, ces deux activités répondent à des logiques et à des finalités distinctes. Rien n'empêche cependant que les deux missions soient prises en charge par une même instance, comme c'est le cas pour le conseil scientifique du Réseau des MSH, à condition que la finalité et les modalités des deux soient bien précisées. II est donc important d'utiliser le terme évaluation en faisant référence à l'optique pertinente et à la finalité visée.

Le chapitre commence par un rappel du contexte dans lequel se situent les activités d'évaluation. II analyse ensuite les implications, en matière d'évaluation, de la diversité des statuts des

\footnotetext{
95 - Grunberg G. (sous la dir.), (2003), «Rapport de conjoncture établi à partir des débats et contributions du conseil scientifique de département pour réaliser une synthèse des rapports des sections », p. 28, conseil scientifique du département des sciences de l'homme et de la société, Paris: CNRS (document interne).
} 
Maisons, et du changement de statut du Réseau, transformé en 2006 en groupement d'intérêt scientifique (GIS), avant de passer en revue les différentes formes d'évaluation adoptées dans le cadre du Réseau MSH et d'en relever les implications pour les missions du futur conseil scientifique. Pour terminer, nous faisons le point sur la fonction de l'évaluation dans le contexte du GIS et sur son rôle dans le pilotage de la recherche pluridisciplinaire en SHS.

\section{Un contexte nouveau}

Ce chapitre s'appuie sur l'expérience des membres du conseil d'orientation scientifique (COS) du Réseau des MSH entre 2000 et 2005, expérience qui a suscité des débats au sein du COS sur la question de l'évaluation et de son rôle dans le fonctionnement du futur conseil scientifique à un tournant important du développement du Réseau. Trois grands changements ont contribué à l'actualité de cette réflexion sur l'évaluation.

Tout d'abord la création, en 2005, au niveau national, de l'Agence nationale de la recherche (ANR) a entraîné la suppression du Fonds national de la science (FNS) et a donc mis fin aux financements spécifiques du Réseau par les actions concertées incitatives (ACI), destinées à promouvoir la structuration de la recherche en SHS. Tandis que les $\mathrm{ACl}$ avaient fortement développé l'esprit de Réseau en misant sur des programmes scientifiques partagés, les mobilités et la mutualisation des moyens, les appels d'offres blancs de l'ANR en SHS, par contre, sont ouverts à tous les chercheurs et enseignants-chercheurs dans ces disciplines.

Ensuite le Réseau, qui relevait depuis sa fondation en 2001 d'une convention entre le ministère chargé de la recherche, le CNRS, les présidents des universités de rattachement des Maisons et les 
directeurs des Maisons dotées de structures juridiques auto-

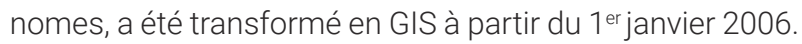

Enfin, la création d'une Agence nationale d'évaluation de la recherche, attendue en 2006 dans le cadre de la loi d'orientation et de programmation de la recherche, prévoit le rapprochement des procédures d'évaluation des organismes, notamment du CNRS et des universités. Ce mouvement a des implications pour l'évaluation des activités du Réseau, puisque la plupart des Maisons dépendent à la fois des universités et du CNRS pour leur évaluation.

\section{La diversité des statuts}

Le statut adopté par les Maisons détermine non seulement leur structure administrative, mais aussi les procédures et modalités d'évaluation auxquelles elles sont soumises.

\section{Le statut d'unité mixte de service (UMS) - treize Maisons}

Le statut d'UMS est de loin celui le plus largement adopté pour les Maisons. Les UMS sont des structures qui proposent des services d'appui à la recherche, autour d'une technique ou d'un savoir-faire (cartes, construction de bases de données, laboratoire, analyse). Ce sont des entités pilotées conjointement par le CNRS et un établissement d'enseignement supérieur. Elles abritent le plus souvent des personnels CNRS (ingénieurs, techniciens et administratifs, ITA) et universitaires (ingénieurs, agents administratifs, techniques, ouvriers de services sociaux, de santé et de bibliothèques, IATOS), mais peu de chercheurs et d'enseignants-chercheurs. La formule souple d'UMS sert de canal technique pour l'affectation de moyens scientifiques et humains, sans rien indiquer sur la programmation scientifique des Maisons, ni demander leur évaluation par le CoNRS en tant qu'entité de recherche. 


\section{Le statut de fédération de recherche (FR) - la}

\section{Maison de l'Orient méditerranéen (Lyon MOM)}

Les FR sont des groupements de structures de recherche relevant du CNRS et d'autres organismes, en vue de coordonner leurs activités scientifiques et la mise en commun des moyens, en principe sur un même site. Elles n'ont pas vocation à fusionner les programmes de recherche des unités fédérées. Puisque la plupart des FR résultent d'un partenariat entre le CNRS et des établissements d'enseignement supérieur ou des organismes publics, elles regroupent plusieurs unités mixtes de recherche (UMR) et financent, en général, des projets transversaux. Les FR sont créées pour quatre ans renouvelables et sont soumises pour évaluation aux instances compétentes du Comité national de la recherche scientifique, à l'instar des unités de recherche composantes. Le statut de FR est assez bien adapté à la mission des Maisons dans la mesure où il leur permet de conduire des activités de recherche propres, tout en préservant l'autonomie des unités constitutives.

\section{Le statut de fondation d'utilité publique - la MSH de Paris}

Le statut de fondation d'utilité publique, structure adoptée par la première des MSH, celle de Paris, fondée en 1963 par Fernand Braudel pour favoriser un mode de structuration de la recherche en dehors des disciplines, lui permet de bénéficier d'une grande autonomie. Les activités propres de la fondation sont évaluées par la Mission scientifique, technologique et pédagogique (MSTP), dans le cadre de la contractualisation. En 1993, la MSH a mis en place un comité international d'évaluation scientifique, qui tient lieu, en quelque sorte, de conseil scientifique pour les programmes de la fondation. 


\section{Le statut de groupement d'intérêt public (GIP) - la Maison des sciences de l'Homme "Ange-Guépin » à Nantes}

Les GIP sont toujours constitués d'au moins deux personnes morales de droit public, dont un établissement public ayant une activité de recherche ou de développement technologique. Ils doivent aussi compter au moins un partenaire non public. Ils visent à regrouper sur un objectif précis ou sous une thématique de recherche différents partenaires institutionnels. Leur objet consiste à mettre en commun des moyens pour l'exercice d'activités de recherche ou de développement technologique, ou pour la gestion d'équipements d'intérêt commun, nécessaires à ces activités. Ils ne relèvent pas du CNRS, bien que celui-ci puisse y être impliqué. En contrepartie de la personnalité qui lui est reconnue, la création du GIP est soumise à un formalisme lourd et contraignant. Sa constitution nécessite, en effet, des délais souvent longs, puisque le conseil d'administration du CNRS délibère sur la création, les modifications statutaires et le renouvellement des GIP, et qu'un arrêté interministériel clôture la procédure. La majorité des droits statutaires doit être détenue par le secteur public. Enfin, le GIP est nécessairement soumis à un contrôle des pouvoirs publics, impliquant la désignation d'un commissaire du gouvernement, d'un contrôleur d'État et d'un contrôle a posteriori de la Cour des comptes. Le GIP «Ange-Guépin » est doté d'un conseil scientifique dont les membres sont nommés avec l'accord du CNRS.

Le statut d'association de la loi de 1901 - la Maison des sciences de l'Homme d'Aquitaine

Créées dans le cadre de la loi de 1901, les associations ne relèvent pas d'un établissement public de recherche. La Maison d'Aquitaine est un dispositif de recherche en sciences humaines et sociales à 
vocation interdisciplinaire. Elle est dotée d'un contrat quadriennal spécifique articulant un ensemble de programmes en SHS, ouvrant ainsi la possibilité d'être évaluée par la MSTP dans le cadre de la contractualisation.

\section{Le statut de groupement d'intérêt scientifique (GIS) - I'Institut international Erasme à Lille ainsi que le Réseau MSH à partir de 2006}

Le GIS est une structure qui vise, comme le GIP, à regrouper sur une thématique de recherche ou sur un objectif précis plusieurs partenaires institutionnels. À la différence du GIP, le GIS n'a pas de personnalité morale. En conséquence, il ne dispose pas des facilités qu'ouvre l'autonomie financière et juridique pour gérer des moyens, exécuter des travaux ou recruter du personnel. II a donc besoin d'être adossé à une entité juridique, telle qu'un établissement d'enseignement supérieur ou un organisme de recherche. Les équipes conservent leur individualité, et les personnels appelés à travailler en leur sein restent affectés à l'unité dont ils relèvent. Aucun patrimoine propre ni recrutement de personnel ne sont possibles. Un GIS peut se munir d'une convention pour créer des organes particuliers, destinés à veiller au bon déroulement de la coopération, tels un conseil de gestion et un conseil scientifique, conformément à la convention constituant le Réseau.

Comme elle n'a pas besoin d'être soumise à l'avis des instances compétentes du CoNRS, la création du GIS présente l'avantage d'être souple et rapide. Sa durée est laissée à la libre appréciation des parties. Le directeur du GIS est désigné par les parties et ses fonctions sont différentes de celles confiées à un directeur chargé de la responsabilité scientifique et administrative d'une structure CNRS. En revanche, le GIS bénéficie a priori de peu de garanties scientifiques et son évaluation n'est pas, en principe, du ressort du CoNRS. 


\section{La mixité des statuts à l'intérieur des Maisons}

La plupart des Maisons sont composées d'UMR et d'équipes universitaires. Les UMR sont des regroupements de recherche qui accueillent aussi bien des chercheurs CNRS que des enseignants-chercheurs, et dont le pilotage scientifique est assuré conjointement par le CNRS et l'établissement d'enseignement supérieur cocontractant. Tandis que les équipes universitaires sont évaluées par la MSTP, l'évaluation des UMR est assurée par le Comité national de la recherche scientifique (CoNRS) pour le compte de tous les partenaires institutionnels cocontractants. Exceptionnellement, la MSTP intervient si l'évaluation par le CoNRS n'est pas en phase avec le contrat quadriennal.

II est courant que les structures fédératives se dotent d'instances d'orientation et d'évaluation (conseil scientifique), mais lorsqu'il y a superposition de structures de ce type, il n'y a pas toujours articulation et coordination entre les partenaires cocontractants. Dans le cas des MSH par exemple, certaines Maisons sont inscrites comme composante de l'université qui les accueille physiquement, au même titre qu'un département, un laboratoire et un centre de recherche. Le fait d'être à la fois UMS pour le CNRS et composante pour l'université peut ainsi donner lieu à une coexistence de statuts et à une diversité de règles de fonctionnement.

\section{À chaque source de financement, son évaluation}

Théoriquement, il peut y avoir évaluation dès qu'il y a attributions de crédits, qu'il s'agisse de crédits contractualisés ou de crédits attribués pour des recherches ciblées sur programmes. Au cours d'une période de quatre ans, les structures de recherche d'une Maison et ses diverses composantes sont ainsi évaluées par la MSTP (équipes universitaires, écoles doctorales) et par le CoNRS 
(équipes associées et UMR) dans le cadre de la négociation du contrat quadriennal. Elles peuvent aussi être évaluées dans le cadre d'un financement européen, par un comité international. S'y ajoutent les évaluations demandées par les collectivités territoriales, lorsqu'elles soutiennent des programmes scientifiques conduits par les Maisons (dans ou hors contrats de plan ÉtatRégions), et les évaluations liées aux appels d'offres de l'ANR, lorsque la Maison ou ses chercheurs répondent à ces appels d'offres. Les visites de l'Inspection générale de l'administration de l'Éducation nationale et de la Recherche ${ }^{96}$ et de la Cour des comptes sont également vues par les intéressés comme autant d'évaluations.

Aux yeux des Maisons, les évaluations les plus importantes sont celles qui assurent leur pérennité, c'est-à-dire celles où le lien entre évaluation et financements contractualisés est univoque. La contractualisation des Maisons n'est cependant pas systématique et paradoxalement, lorsqu'une Maison apparaît dans le contrat quadriennal d'une université, ce n'est souvent que par le biais d'un programme pluri-formation (PPF) et non comme structure effective de recherche.

II en ressort que les composantes des Maisons sont soumises individuellement et collectivement à de multiples évaluations, en raison des diverses sources de financement dont elles disposent, en général sans que les évaluations soient coordonnées. Afin

96 - IGAENR (Inspection générale de l'administration de l'Éducation nationale et de la Recherche), (2004), Les Maisons des sciences de l'Homme (MSH), rapport par S. Srodogora, G. Lesage, J.-R. Cytermann, M. Tyvaert et T. Chalon au ministre de l'Éducation nationale, de l'Enseignement supérieur et de la Recherche, et au ministre délégué à la Recherche, n²004-141, Paris: IGAENR. ftp : //trf. education. gouv. fr/pub/edutel/syst/igaen/rapports/msh_04. pdf 
d'éviter des charges supplémentaires par la multiplication des évaluations, il importe donc de poser la question de leur finalité et de leur valeur ajoutée.

\section{Missions du nouveau conseil scientifique du Réseau}

Répondant à une préoccupation scientifique, la création du Réseau des MSH sollicitait une organisation plus structurée que celle applicable dans un contrat classique. La première convention en 2001 a ainsi institué les instances destinées à veiller au bon déroulement de la coopération sous forme d'un conseil de gestion et d'un conseil scientifique. Les activités qui ont été évaluées jusqu'en 2005 par le cOS le seront désormais par le conseil scientifique du GIS. La mission de celui-ci reste en effet précisée dans la charte : il « évalue, au regard des obligations qu'elles ont souscrites en adhérant à la charte, l'activité des MSH, il évalue l'activité du Réseau et il se prononce sur les demandes d’adhésions nouvelles au Réseau ».

II revient donc au conseil scientifique de se prononcer sur les demandes d'adhésion et sur les performances des MSH, aussi bien au niveau des Maisons qu'au niveau du Réseau et de transmettre ses avis au comité directeur de celui-ci.

Compte tenu du nouveau contexte du pilotage de la recherche en France et des expériences acquises dans le cadre du COS, la convention du GIS apporte les précisions suivantes sur le rôle du conseil scientifique du GIS « MSH ». II doit :

- formuler un avis sur les orientations générales et les priorités du Réseau ;

- donner son avis sur les demandes d'adhésion au Réseau qu'il évalue conformément à la charte ; 
- élaborer une synthèse annuelle à partir des rapports d'activité qui lui sont adressés par le conseil scientifique de chacune des Maisons, conformément à la charte ;

- formuler un avis préalable à la décision du comité directeur sur l'exclusion d'une Maison.

La charte et la convention du GIS attribuent donc au conseil scientifique une fonction d'évaluation et de pilotage des activités, tant au niveau des Maisons qu'à celui du GIS. Selon la nature des objectifs visés, les activités d'évaluation peuvent être perçues tantôt comme outils de gestion, servant à assurer un choix optimal de projets et d'actions à promouvoir en fonction des priorités et stratégies affichées, tantôt comme instruments stratégiques de pilotage aussi bien pour les activités de recherche que pour les actions incitatives.

Durant son mandat, le COS a progressivement élaboré et rendu opérationnelle une réflexion sur les procédures pour formuler et transmettre ses avis au comité directeur du Réseau. Pendant les quatre premières années de son mandat, le Réseau a reçu un nombre important de demandes d'adhésion de nouvelles Maisons. Bénéficiant à cette époque d'un financement fléché par le biais d'une $\mathrm{ACl}$, le COS a eu pour tâche principale d'évaluer les projets scientifiques de Maisons souhaitant rejoindre le Réseau, ainsi que les réponses aux appels d'offres dans le cadre des $\mathrm{ACl}$, effectuées en liaison avec la MSTP.

La majeure partie du travail d'évaluation du COS a eu pour objectif d'assurer l'adéquation des moyens mis en œuvre et, à un moindre degré, l'accompagnement et le suivi des Maisons à but proactif et formatif. Les activités d'évaluation ont donc été réalisées essentiellement dans une perspective ex ante, visant à formuler des 
recommandations concernant les projets de nouvelles Maisons ou des avis sur les projets soumis en réponse aux appels d'offres. Le Cos n'a pas été interpellé pour faire des évaluations a posteriori ou se prononcer sur les résultats des recherches financées. II n'a pas entrepris de vérifications des «performances » par rapport aux objectifs visés. II n'a pas non plus eu à statuer sur la valorisation des travaux, et aucun cas de sortie du Réseau par une Maison n'a été appréhendé.

Dans le cadre du GIS, l'essentiel de l'activité du conseil scientifique devrait désormais porter à la fois sur le suivi des Maisons et leur positionnement par rapport au Réseau, et sur les orientations prioritaires et stratégiques du Réseau à promouvoir. Bien qu'il soit possible que celui-ci finance ses propres appels d'offres et que l'on s'adresse au conseil scientifique pour évaluer les dossiers, et sous réserve également de nouvelles demandes d'adhésion au Réseau, la mission d'orientation stratégique est, de ce fait, dorénavant appelée à prendre toute son ampleur.

\section{Instruments et procédés d'évaluation du conseil d'orientation scientifique}

Les activités du conseil scientifique en matière d'évaluation s'inscrivent dans trois catégories d'instruments incitatifs et de pilotage dont les modalités ont été développées par le COS :

- autorisation du label MSH ;

- sélection des projets dans le cadre d'appels d'offres ;

- suivi des performances, au niveau des Maisons et au niveau du Réseau.

\section{Le label MSH}

L'objectif principal de la charte des MSH est de définir les missions des Maisons qui souhaitent être reconnues comme telles. Elle 
conditionne la reconnaissance d'une Maison en tant que MSH à sa capacité à définir et à mettre en œuvre un projet scientifique auquel participent des équipes, des chercheurs et des enseignants-chercheurs rattachés à la Maison. Ces missions sont précisées dans les termes suivants:

- regroupement de chercheurs, équipes ou unités de recherche relevant de disciplines différentes, associées dans la conduite de projets scientifiques ;

- promotion d'une interdisciplinarité active avec des programmes communs ;

- définition d'une identité scientifique, qui donne à chaque Maison sa cohérence et lui permet de devenir un pôle de référence à l'échelle nationale et internationale ;

- capacité à innover, que ce soit en matière de thématiques, de programmes ou de chercheurs, équipes ou unités ;

- intégration prioritaire de jeunes chercheurs ;

- intégration internationale ;

- accès à des équipements et à des moyens collectifs ;

- collaboration avec des institutions publiques et privées ;

- activités de diffusion.

Tous ces critères, et plus particulièrement les projets scientifiques proposés, conditionnent l'acceptation par le conseil d'orientation des projets de nouvelles Maisons, en vue de leur adhésion au Réseau. Celles-ci doivent ensuite se doter d'un conseil scientifique qui leur est propre. Il est parfois constitué à l'image des conseils scientifiques des universités, c'est-à-dire avec des représentants des personnels et des représentants de partenaires régionaux. 


\section{Procédures d'admission au Réseau}

Les premières demandes d'admission au Réseau provenaient des projets de nouvelles Maisons à Strasbourg, Paris-Nord (Saint-Denis), Nice, Lille et Montpellier. Chaque dossier, analysé par le COS, était présenté devant le conseil par le chef de projet accompagné de son président d'université. Certains projets jugés insatisfaisants en l'état ont fait l'objet de recommandations et d'un accompagnement par le COS, deux de ses membres étant choisis comme experts pour une visite sur place. Les projets scientifiques ont ensuite été validés au fur et à mesure que les échanges avec le coS permettaient aux porteurs des projets de mieux répondre aux critiques formulées initialement.

Par la suite, les procédures d'admission des Maisons sont devenues plus formalisées. Lorsqu'un projet est soumis au COS, son président et le président du conseil des directeurs se rendent sur place pour une prise de contact avec les différents acteurs du projet. Parallèlement, le conseil d'orientation nomme deux de ses membres (un Français et un étranger) comme rapporteurs pour effectuer une visite du site et pour examiner le projet scientifique avec les équipes impliquées, le(s) président(s) de l'(des) université(s) et le cas échéant d'autres partenaires. Les rapporteurs rédigent ensuite un avis et le dossier est soumis au COS qui en débat, les représentants du département SHS du CNRS et de la Direction de la recherche étant systématiquement invités aux réunions du conseil. Conformément aux statuts du Réseau, l'avis du COS est ensuite transmis au conseil des directeurs qui statue sur l'admission ou non de la future Maison dans le Réseau.

Les critères scientifiques pour l'évaluation des projets sont les critères habituels: définition de l'objet de recherche, objectifs, méthodes et calendrier proposés, moyens demandés, auxquels 
s'ajoute la pertinence du projet dans les axes de la Maison. La nécessité d'identifier un nombre limité d'axes est particulièrement importante mais difficile à mettre en œuvre. Elle est importante pour la viabilité du projet de Maison, mais difficile à réaliser, car source d'exclusion des équipes qui n'entrent pas dans les axes choisis, et donc source de tensions contraires à la volonté fédératrice d'une Maison au niveau local.

\section{Observations sur les demandes d'admission au Réseau} À travers l'expérience de quatre années de fonctionnement, le cos a relevé, comme étant essentielles pour la genèse et la réussite d'une Maison, les conditions suivantes:

- la présence d'un chef de projet scientifique, choisi en accord avec l'ensemble des universités appelées à se mobiliser pour la future Maison;

- la cohérence d'un projet scientifique autour d'un petit nombre d'axes de recherche, donnant au projet de Maison une identité scientifique et une visibilité locale et nationale, impliquant une bonne définition, au départ, de l'état de l'art dans les domaines de recherche concernés ;

- la présence du CNRS et du ministère dans le développement du projet de Maison ;

- l'existence de locaux et d'équipements de recherche adaptés (construction ou réhabilitation);

- la présence d'une stratégie concertée pour assurer une interdisciplinarité productive (par exemple par des approches pluridisciplinaires d'un même objet de recherche) ;

- des mesures prévues pour assurer l'insertion de jeunes chercheurs et la place accordée à la formation par la recherche et l'accueil des doctorants dans la Maison; 
- l'identification, pour chacun des axes, des projets de recherche soutenus par la Maison, ainsi que des responsables des projets et des équipes participantes, y compris les collaborations internationales ;

- les efforts consentis pour assurer une ouverture au monde socio-économique dans le projet ;

- la prévision de procédures pour assurer le renouvellement des projets et, le cas échéant, une évolution des axes de la Maison.

En se portant garant du label MSH, le Réseau et son conseil scientifique ont aussi en charge de veiller à ce que les Maisons respectent les modalités de fonctionnement inscrites dans la charte. Les Maisons existantes créées antérieurement à la charte sont en principe tenues de faire évoluer leurs pratiques pour se conformer aux principes de la charte. Ainsi la non-conformité à ces objectifs doit pouvoir servir de référence pour une opération de retrait ou d'exclusion d'une Maison.

Une leçon à tirer de l'expérience du cos est que le travail relatif aux demandes d'adhésion et aux sorties du Réseau suppose une continuité dans l'accompagnement des Maisons. Ce constat justifie le dispositif des « correspondants » mis en place par le COS, de même que la nomination de membres du conseil scientifique, chargés de suivre la conception et la réalisation des projets de création de nouvelles Maisons et leur insertion ultérieure dans le Réseau.

\section{Les appels d'offres}

Le Réseau des MSH a pu bénéficier, dès 1999, d’une ACl lui permettant d'assurer sa structuration. Les dossiers de candidature 
préparés par les Maisons devaient être fondés sur des projets scientifiques représentant leurs axes ou thèmes prioritaires, de façon à favoriser l'émergence ou le développement de spécificités et donc d'une identité.

Par la suite, d'autres $\mathrm{ACl}$ ont favorisé les rapprochements entre les Maisons. Dans le cadre de l'ACl « Terrains, techniques et théories » (TTT), les propositions faites par les Maisons ont permis de renforcer l'aspect fédérateur du Réseau par la mutualisation de moyens techniques et la mise en commun de projets scientifiques, pour certains, nouveaux et innovants. Les $\mathrm{ACl}$ ont ainsi servi d'instrument pour consolider les activités coopératives entre les Maisons.

\section{Critères de sélection}

À la différence des autres programmes lancés par le FNS, les ACl dédiées aux MSH ont pris en compte des critères qui ne sont pas exclusivement scientifiques. Le conseil a ainsi élaboré une fiche d'évaluation précisant les critères suivants :

- qualité scientifique du projet (contenu, aspect novateur) ;

- cohérence avec les axes scientifiques des MSH participantes ;

- projet structurant pour les collaborations scientifiques entre $\mathrm{MSH}$;

- qualité des partenaires (dont écoles doctorales et, éventuellement, partenaires étrangers) ;

- perspectives de développements ultérieurs (à quoi il convient d'ajouter la valorisation des travaux et le transfert des connaissances) ;

- qualité du dossier (état de la question, hypothèses de travail, méthodologie proposée, cahier des charges faisant mention des moyens à mettre en œuvre, types de réunions, calendrier) ; 
- dimension interdisciplinaire du projet ;

- dimension européenne du projet.

Dans les évaluations rendues, les critères n'ont pas été pondérés et n'ont pas non plus pris en compte la valorisation des travaux déjà financés (mesurée par la production scientifique et le transfert des connaissances provenant de la production scientifique antérieure). En outre, les appels d'offres ne précisaient pas le montant maximal du budget attribué aux projets. Une telle précision aurait pu éviter que les porteurs de projets surévaluent leurs budgets, et que ceux-ci ne soient coupés par la suite.

Pour chaque dossier, le conseil d'orientation a désigné deux de ses membres comme rapporteurs. Ceux-ci ont utilisé une grille de lecture commune, commentant les aspects scientifiques du dossier et offrant une appréciation globale selon les critères retenus. Chaque dossier a été évalué en parallèle par deux experts désignés par la MSTP. Lors de la réunion du conseil, les expertises de la MSTP et celles des rapporteurs du COS ont été prises simultanément en considération afin d'arriver à un avis consensuel. Une synthèse des appréciations a été communiquée aux porteurs de projets.

\section{Observations relatives aux procédés de sélection}

L'expérience a montré l'intérêt et l'importance des $\mathrm{ACl}$ pour le Réseau. Lors de l'ACl «TTT», par exemple, il a été constaté que, quand la réponse aux appels d'offres comportait la construction de réseaux internationaux pour la réalisation d'un programme de recherche spécifique, ce réseau a perduré au-delà du programme. Il s'est développé ensuite sur d'autres thèmes et pour d'autres cycles d'échanges. La dimension « internationale » des actions du Réseau des MSH ne s'est, en effet, pas limitée à la participation 
occasionnelle de chercheurs invités. II n'est pas exclu que, à l'avenir, le Réseau s'organise pour faire ses propres appels à projets afin de consolider les liens entre les Maisons. Dans cette optique, il peut être intéressant de tirer les leçons de l'expérience du COS pour que ce type d'évaluation se fasse dans les meilleures conditions possibles.

L'examen des dossiers en réponse aux appels d'offres soulève de nombreuses questions sur le fonctionnement du système d'évaIuation par les pairs, questions qui se posent quels que soient le dispositif et l'objet de l'évaluation. Puisque la légitimité et la crédibilité du conseil scientifique en dépendent, il serait sans doute utile de susciter un débat sur les principes déontologiques de l'évaluation et sur leur mise en œuvre.

Ces principes sont nombreux ${ }^{97}$, les plus pertinents pour le Réseau étant sans doute les suivants :

- assurer par la composition de l'instance d'évaluation, la représentativité scientifique (et non une représentativité par groupes d'intérêts), la diversité des disciplines et des approches méthodologiques, l'extériorité (par la présence d'étrangers et de personnalités non impliquées dans le fonctionnement des Maisons), et l'intégrité (assurée par l'application de principes déontologiques consacrés par le désintéressement et la séparation des pouvoirs);

97 - CNER (Comité national d'évaluation de la recherche), (2003), Évaluation de la recherche publique dans les établissements publics français, Paris : La Documentation française, http : //www.cner.gouv.fr

COMETS (Comité d'éthique du CNRS), (2004), "Éthique et évaluation », juin (document interne). Conseil national du développement des sciences humaines et sociales, (2001), Pour une politique des sciences de l'homme et de la société : recueil des travaux (1998-2000), Paris: Presses universitaires de France/Quadrige. 
- appliquer dans les procédures d'examen et de sélection de projets des principes de transparence, d'objectivité et d'impartialité ;

- assurer la transparence par la communication explicite des objectifs, des indicateurs et des critères, ainsi que par la communication des résultats ;

- veiller à la pertinence et à l'efficience du processus par des suivis systématiques.

Ces mêmes principes veulent que les experts déclarent tout conflit d'intérêt et que, lors du lancement des appels d'offres, les membres du conseil et les porteurs de projet soient informés des critères prioritaires qui seront utilisés et de leur pondération. Ils veulent aussi que les personnes intéressées par le projet ne soient pas présentes lors des délibérations, que les évaluateurs fassent preuve d'objectivité et d'intégrité et que leur compétence en la matière soit reconnue.

Le conseil n'étant pas un comité d'évaluation censé posséder les connaissances scientifiques nécessaires pour évaluer tous les aspects de tous les dossiers qui lui sont remis, il devrait faire systématiquement appel à des experts externes choisis pour leur compétence scientifique ou technique dans le domaine en question. Le nombre d'experts doit pouvoir varier en fonction du type de financement à engager et de la nature des dossiers à traiter.

En comparaison à d'autres conseils scientifiques en France, le conseil scientifique du Réseau a la particularité d'être composé d'une proportion importante (le tiers) d'étrangers francophones, susceptibles de porter un regard extérieur sur les activités des Maisons et sur leurs orientations stratégiques. Convaincu de l'intérêt de leur contribution en raison de leurs connaissances et de 
leurs compétences différentes, le COS a su bénéficier de son ouverture vers l'extérieur et de l'apport des étrangers pour mettre en valeur la diversité des approches culturelles et pour nourrir l'échange des bonnes pratiques déontologiques.

Le Comité d'éthique du CNRS (COMETS, 2004) souligne l'importance de l'ouverture à des évaluateurs étrangers comme moyen d'élargir la base de compétence et de jugement des instances d'évaluation. Cependant, il émet certaines mises en garde à ce propos. Même si la présence de membres étrangers participe à la légitimité des travaux du conseil, le COMETS reconnaît qu'elle n'est pas automatiquement un gage de neutralité et d'indépendance. Ce constat s'applique au conseil scientifique du Réseau des MSH, puisque la francophonie de ses membres est due souvent au fait qu'ils sont, d'une manière ou d'une autre, impliqués dans le milieu français de la recherche.

Sans que le Réseau ait bénéficié d'une évaluation formelle par le COS, le bilan de l'activité du COS confirme l'intérêt de sa composition internationale pour une vision distanciée de l'activité du Réseau et une mise en perspective internationale de celle-ci.

\section{Le suivi}

Les activités de suivi ont été développées dans le prolongement des évaluations de demandes d'adhésion des nouvelles Maisons, avec la désignation pour chaque Maison de deux correspondants, membres du conseil, chargés par le COS de suivre l'évolution des projets scientifiques de celle-ci. Ce dispositif a une double finalité, pour les Maisons et pour le Réseau.

Pour les Maisons, il est utile comme :

- outil pour dialoguer et faciliter l'inscription active des Maisons dans une stratégie collective au niveau du Réseau ; 
- outil d'autorégulation, permettant aux Maisons d'ajuster plus facilement leur action et leurs priorités en fonction d'un regard extérieur.

Sur le plan du Réseau, le suivi des Maisons par le conseil scientifique se présente comme un outil stratégique de gestion, nécessaire pour mener une action concertée. II constitue :

- un instrument d'accompagnement, permettant au conseil scientifique de proposer des mesures visant à contrebalancer les déséquilibres scientifiques ou d'implantation territoriale, ou des problèmes de gestion, par des interventions adaptées, tant au niveau du Réseau qu'à celui des Maisons ;

- un instrument de veille prospective et stratégique, permettant au conseil scientifique d'acquérir une vue d'ensemble des orientations des Maisons, des moyens investis, des lacunes et des points forts ainsi que des problèmes structurels, en référence aux objectifs de la charte et aux principes fondateurs ;

- un instrument de contrôle administratif, permettant au conseil scientifique de se porter garant du label MSH.

\section{Procédés de suivi}

Suite aux visites des deux membres du Cos à l'occasion des demandes d'adhésion, et aux échanges qu'elles ont rendus possibles, échanges vécus très positivement aussi bien par les Maisons que par les experts, le COS a décidé de désigner pour chaque Maison, nouvelle ou ancienne, deux correspondants chargés d'assurer le suivi de la Maison par des visites récurrentes.

L'expérience acquise a montré que les visites pouvaient être doublement instructives et appréciées. Pour les correspondants, notamment les étrangers, il a été très important d'acquérir, lors de 
ces visites, une vue d'ensemble des activités scientifiques des Maisons concernées, de voir les installations et les ressources humaines et matérielles sur place, d'apprécier les mesures de valorisation et d'insertion locale et régionale adoptées par la Maison, et de mesurer l'esprit d'équipe et le climat collectif transmis par les chercheurs.

Pour les Maisons, l'intérêt des visites tient sans doute non seulement aux informations et aux conseils qui ont pu être apportés par les correspondants, mais tout autant aux questions posées par des personnes venant de l'extérieur, engagées dans l'avenir de la Maison et dans sa stratégie, mais avec une vue distanciée. Un autre facteur à prendre en compte est l'effet mobilisateur de la participation collective des équipes dans une présentation commune de l'ensemble des activités de la Maison, facteur ayant d'autant plus d'effets si un dialogue en commun avec toutes les équipes peut être organisé pendant la visite.

\section{Observations sur la fonction de suivi}

La finalité du suivi dans le cadre du GIS est double : elle se situe, d'une part, au niveau du Réseau et, de l'autre, au niveau de chaque Maison, prise individuellement. Dans les deux cas, il constitue un instrument de pilotage essentiel.

Dans la convention de création du GIS, le suivi des Maisons se situe dans le cadre de bilans annuels. Cette convention charge en effet le conseil scientifique de faire un bilan annuel à partir de rapports d'activités, fournis par les Maisons. La mission du conseil scientifique consistant à dresser une synthèse à partir de ces rapports d'activités permet au GIS d'avoir une vue d'ensemble des activités en cours, et de s'en servir pour nourrir un débat prospectif. 
Certains des principes qui se dégagent de l'expérience du COS sont à retenir pour le développement d'un pilotage dynamique :

- adopter une logique d'accompagnement, basée sur un échange d'informations pertinentes, en aval et en amont, et un dialogue portant sur les problèmes perçus et les choix stratégiques, tant au niveau des Maisons qu'au niveau du Réseau ;

- bien expliquer aux Maisons le sens des visites des correspondants délégués et les attentes du conseil scientifique;

- viser une concertation et une coordination avec l'agenda des Maisons et de leurs conseils scientifiques, afin d'éviter la multiplication des visites et des demandes administratives faites aux Maisons par les différents acteurs intéressés ;

- constituer, pour les évaluations a posteriori des performances scientifiques d'une Maison donnée, une instance légère de coordination, permettant de convenir de l'organisation et de la périodicité de visites communes avec d'autres instances d'évaluation.

Les rapports annuels que doivent élaborer les conseils scientifiques des Maisons permettent aux correspondants d'assumer plus activement une fonction de relais pour l'inscription active des Maisons, non seulement dans le Réseau en tant que tel, mais également dans d'autres réseaux, nationaux et internationaux. Les correspondants deviennent, dans ce contexte, plutôt des « ambassadeurs ».

Dans le cadre du GIS, la procédure légère d'évaluation, proposée ci-dessus, devrait permettre au GIS non seulement d'élaborer la synthèse annuelle des rapports d'activité des Maisons, mais aussi d'évaluer les résultats des financements accordés et de l'action de 
valorisation accomplie par la Maison, en tenant compte, entre autres, de la conformité aux objectifs visés, de la valeur ajoutée pour le Réseau, des réalisations interdisciplinaires, de la communication des résultats, et des coopérations et financements acquis au niveau national et international.

\section{L'évaluation comme instrument de pilotage : bilan et perspectives}

Le bilan présenté dans cet ouvrage fait ressortir l'utilité du travail de soutien, de conseil et d'accompagnement dans le processus de création des Maisons, grâce aux échanges avec les promoteurs des projets, aux missions sur les sites de membres du COS, et à la mise en place de correspondants pour chacune des Maisons existantes. II montre, en outre, l'utilité du rôle d'« ambassadeur » joué par les membres du COS pour convaincre tous les acteurs concernés de l'intérêt des principes fondateurs des Maisons, tels qu'ils sont contenus dans la charte. À cela s'ajoute une fonction de promotion pour faire rayonner, au niveau national et international, cette politique de structuration de la recherche en SHS.

Le contexte nouveau dans lequel le conseil scientifique du Réseau va prendre ses fonctions, contexte rappelé au début de ce chapitre, donne au conseil l'opportunité de développer une réflexion sur les orientations stratégiques du Réseau. Dans le cadre du GIS, le conseil scientifique est bien placé pour assumer une fonction spécifique d'évaluation qui se distingue de celle portant sur les équipes et leurs programmes de recherche. L'évaluation des équipes se fait, et continuera à se faire, ailleurs. Les admissions et sorties du Réseau sont peu fréquentes et demandent des dispositifs ad hoc. Les activités de suivi des Maisons rentrent ainsi dans le cadre de la gouvernance 
scientifique, qui relève d'une équipe d'évaluateurs capables d'apprécier aussi bien la qualité des montages interdisciplinaires que l'efficacité et la faisabilité des programmes par rapport à l'environnement local et l'état des connaissances nationales et internationales.

La mission du conseil scientifique, en tant que garant du label MSH, portera davantage sur le suivi des actions engagées, en vue de veiller à la cohérence interne du Réseau, à son ouverture et à son rayonnement au niveau local, national et international. Par les ressources dont il dispose en capital humain et par les synergies qu'il mobilise, le Réseau a vocation à répondre largement aux besoins sociaux de connaissances en SHS. Cette mission privilégie l'ouverture des Maisons sur l'environnement socio-économique et culturel; elle assure en même temps le transfert et la valorisation des connaissances acquises dans un contexte territorial précis, faisant valoir leur inscription au niveau local.

La qualité scientifique des activités de recherche induit bien sûr aussi leur inscription au niveau national et européen. Ce sont autant de critères qui peuvent être utilisés pour évaluer le fonctionnement du Réseau. II reste à les rendre opérationnels dans le dispositif de suivi en élaborant des indicateurs pertinents.

II n'en reste pas moins que les problèmes auxquels sont exposées les Maisons en matière d'évaluation demeurent. Ils sont révélateurs de la façon dont est traitée la question de l'évaluation de la recherche en France:

- la dualité universités-établissements publics de recherche favorise la juxtaposition des évaluations ; 
- la complexité des rapports central-local ne va pas toujours dans le sens d'une coordination des évaluations entreprises à différents niveaux;

- la lourdeur des évaluations ex ante contraste avec l'absence fréquente d'évaluations a posteriori et de suivis.

Plus particulièrement pour ce qui concerne directement les MSH :

- le principe de l'interdisciplinarité au fondement de l'activité des Maisons se heurte à des dispositifs d'évaluation qui restent très fortement disciplinaires;

- la distinction entre ce qui relève spécifiquement de l'activité du Réseau et ce qui relève de l'activité propre des Maisons n'est pas toujours facile à établir et, par conséquent, pose le problème des frontières entre les deux types d'activités pour l'évaluation.

L'expérience du conseil d'orientation du Réseau et les solutions qu'il a trouvées pour apprécier et promouvoir l'orientation et la structuration stratégique de la recherche en SHS pourront servir d'exemple au pilotage d'une recherche au service de la société.

Le Réseau des MSH aura ainsi apporté sa contribution « pour l'avènement de la nouvelle culture scientifique en sciences de l'homme et de la société », que les auteurs de l'introduction à cet ouvrage appellent de leurs vœux. 
Avenir de la recherche 


\section{4 \\ Charte du Réseau des Maisons des sciences de l'Homme}

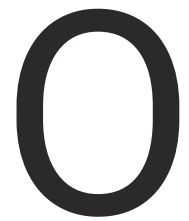

rganisées selon des formules juridiques et universitaires différentes, les Maisons des sciences de l'Homme répondent au même souci de fédérer, structurer et dynamiser la recherche en sciences humaines et sociales. La charte suivante a pour objectif de définir leurs missions communes et de fonder le réseau des Maisons des sciences de l'Homme. Les MSH sont caractérisées par :

- le regroupement en un lieu donné (une maison) de chercheurs, équipes ou unités de recherche relevant de disciplines différentes, associés dans la conduite de projets scientifiques ;

- la promotion d'une interdisciplinarité active, avec notamment des programmes communs à des chercheurs, équipes et centres ;

98 - La charte du Réseau des MSH est consultable sur le site www.msh-reseau.prd.fr. 
- la définition d'une identité scientifique - avec une ou plusieurs dominantes - qui donne à la MSH sa cohérence et lui permet de devenir un pôle de référence à l'échelle nationale et internationale;

- la capacité constamment entretenue de retenir de nouvelles thématiques, d'accueillir de nouveaux chercheurs, de développer de nouveaux programmes et d'être attentif au renouvellement des chercheurs, équipes et unités ;

- l'intégration prioritaire de jeunes chercheurs, doctorants et postdoctorants et, au-delà, la préoccupation des liaisons entre l'enseignement doctoral et la recherche;

- l'intégration internationale des activités de recherche et des chercheurs;

- l'accès à des équipements et à des moyens collectifs ;

- la collaboration avec des institutions publiques et privées ;

- une procédure d'évaluation externe, dans une large mesure internationale.

Le réseau des $\mathrm{MSH}$ est constitué des institutions qui adhèrent à la Charte dont le texte suit.

\section{Article 1}

Les MSH sont des structures de recherche destinées à promouvoir la recherche dans les sciences humaines et sociales. Elles regroupent et accueillent physiquement, en un lieu identifiable, des chercheurs, des équipes et des unités de recherche dépendant de différentes institutions et relevant de différentes disciplines, associés dans la conduite de projets scientifiques. 


\section{Article 2}

Elles développent chacune des activités de recherche propres qui contribuent à définir leur identité scientifique. Elles organisent notamment :

- des programmes de recherches communs à plusieurs disciplines, équipes ou unités de recherche ;

- des initiatives de nature thématique ou méthodologique ;

- l'accueil de programmes temporaires et de jeunes chercheurs dont elles favorisent l'autonomie.

Afin de faciliter le renouvellement des chercheurs et des programmes qui bénéficient de cet accueil, les MSH identifient des surfaces d'accueil temporaire. Elles soutiennent la formation des jeunes chercheurs, notamment au travers des écoles doctorales. Elles veillent à mettre à la disposition des doctorants des moyens de travail en équipement ou surfaces de recherche.

\section{Article 3}

Elles sont reliées entre elles et forment un réseau de communication, d'échanges et de programme scientifique. Le réseau favorise la création de programmes, équipes ou « laboratoires » sans murs.

\section{Article 4}

Elles disposent d'instruments de travail communs aux différentes unités, équipes et programmes de recherche et veillent à leur adaptation permanente. Elles constituent autant de points d'accès au réseau qui assure la mise en commun de ces moyens pour l'ensemble de la communauté scientifique. 


\section{Article 5}

Elles facilitent l'insertion des chercheurs dans la communauté internationale par différentes initiatives telles que, invitations, missions, séminaires, colloques, tables rondes, écoles d'été, projets bi ou multilatéraux.

\section{Article 6}

Les MSH veillent à la meilleure diffusion possible de leurs recherches, auprès des institutions publiques ou privées, à l'échelle locale, nationale ou internationale. Les activités de recherche menées au sein des MSH doivent être identifiées comme telles dans les publications, qu'il s'agisse de recherches propres ou des recherches des unités ou équipes.

\section{Article 7}

Les centres, équipes et unités de recherche accueillis par une MSH continuent à dépendre de leurs propres instances d'évaluation et gardent leur autonomie tant scientifique et administrative que financière, mais ils contribuent aussi au fonctionnement de la MSH qui les accueille.

\section{Article 8}

Chaque MSH est dotée d'un conseil scientifique propre qui assiste la direction de la maison dans la définition de sa politique scientifique. 


\section{Article 9}

Le réseau des MSH est doté d'un conseil scientifique. Garant du label MSH, il veille à maintenir la cohérence du réseau tout en assurant son ouverture. À ce titre, il évalue, au regard des obligations qu'elles ont souscrites en adhérant à la charte, l'activité des MSH, il évalue l'activité du réseau et il se prononce sur les demandes d'adhésions nouvelles au réseau. 


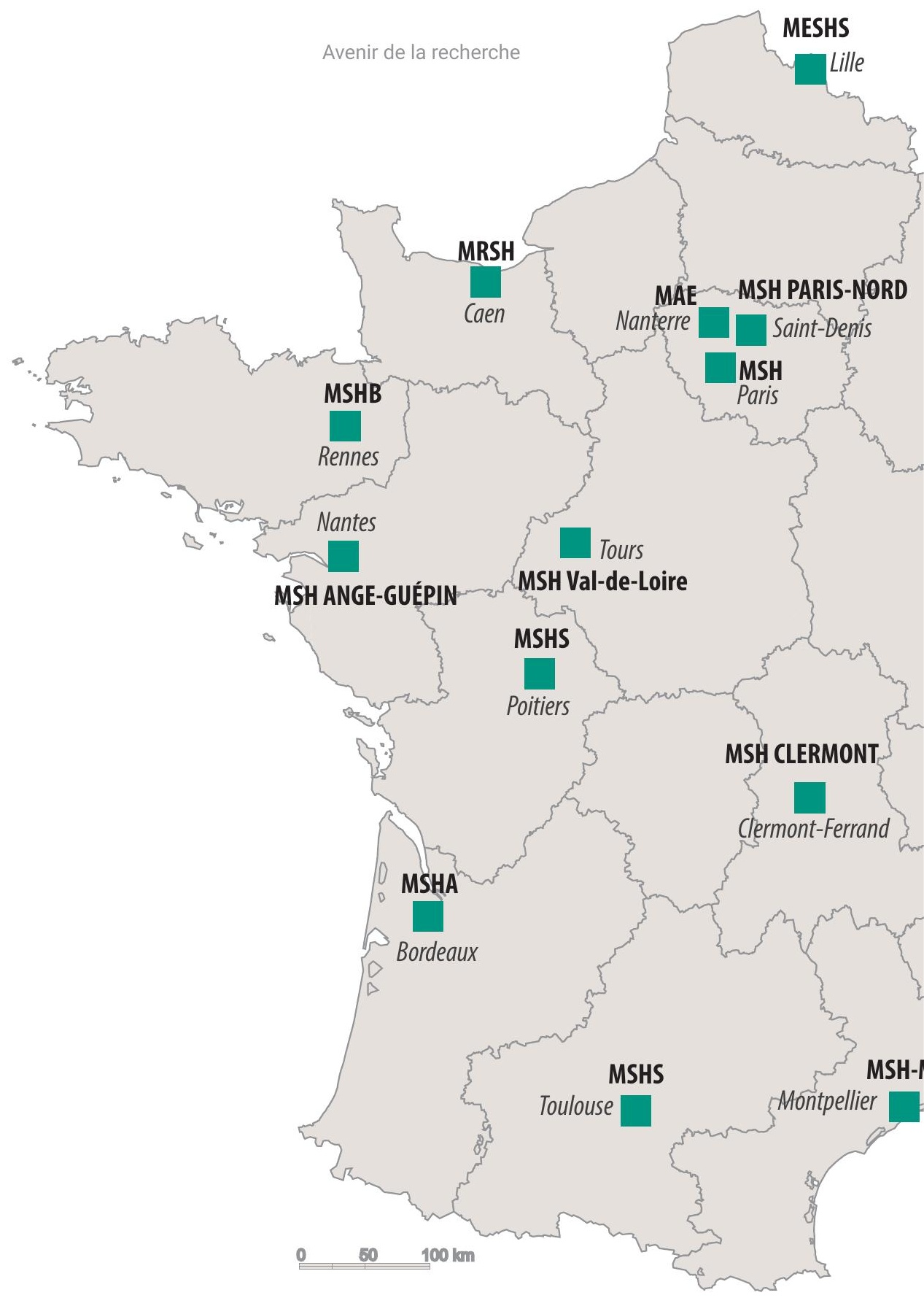




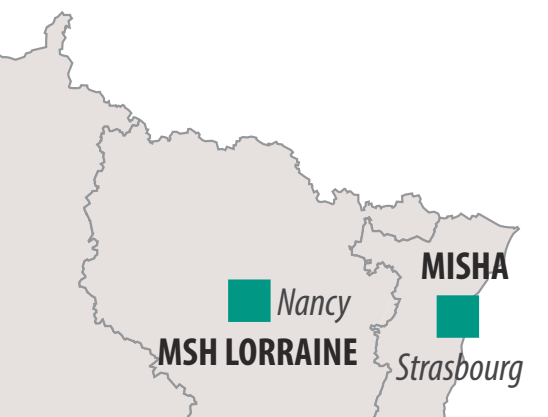




\section{Liste des contributeurs}

Birgit Arve-Parès : (2006) secrétaire scientifique au Conseil suédois de la recherche scientifique, membre du conseil d'orientation du Réseau national des MSH

Martine Bentaboulet : (2006) directrice de recherche au CNRS, chargée de mission du conseil d'orientation du Réseau national des MSH

Pascal Buléon : (2020) directeur de la MrSH de Caen, membre du directoire du Réseau national des MSH

Jacques Commaille : (2006) président du conseil d'orientation du Réseau national des Maison des sciences de l'Homme

Pierre Guibentif : (2006) professeur à I'Instituto Superior de Ciências do Trabalho e da Empresa (ISCTE) et à I'Université nouvelle de Lisbonne, Portugal, membre du conseil d'orientation du Réseau national des MSH

Linda Hantrais : (2006) directrice de l'European Research Centre, Loughborough, UK, membre du conseil d'orientation du Réseau national des MSH

Gilles Pollet : (2020) directeur de la MSH Lyon-Saint-Etienne, membre du directoire du Réseau national des MSH

Pierre Rouillard : (2006) président du conseil des directeurs des Maisons des sciences de l'Homme 
Nicolas Thély : (2020) directeur de la MSHB de Rennes, membre du directoire du Réseau national des MSH

Serge Wolikow : (2006) président du conseil des directeurs des Maisons des sciences de l'Homme 


\section{Bibliographie}

(COLL.), « Sciences de l'Homme et de la Société », Lettre du département $n^{\circ}$ 45, (Paris CNRS), janvier 1997.

(COLL.), « Sciences de l'Homme et de la Société », Lettre du département $n^{\circ} 53$ (Paris CNRS), février 1999.

(COLL.), « Sciences de l'Homme et de la Société », Lettre du département $n^{\circ} 68$, numéro spécial : "Les structures fédératives 》, (Paris, CNRS), août 2003.

(COLL.), Le réseau national des MSH (plaquette réalisée par la Maison des sciences de l'Homme d'Aquitaine, en collaboration avec les autres Maisons membres du Réseau), 2004 (disponible sur http : //www.msh-reseau.prd.fr/; existe également en version anglaise: National Network of social Sciences and Humanities Research Institutes).

(COLL.), Ouvrir les sciences sociales, Rapport de la Commission Gulbenkian pour la restructuration des sciences sociales (présidée par Immanuel Wallerstein), Paris, Descartes et Cie, 1996 (édition originale: Open the Social Sciences, Stanford University Press, 1996).

(COLL.), Re-inventing the Social Sciences, OCDE, 2004.

CNER (Comité national d'évaluation de la recherche), (2003), Évaluation de la recherche publique dans les établissements publics français, Paris: La Documentation française, http : //Www.cner. gouv.fr 
COMMAILLE Jacques et ROUILLARD Pierre, (2004), Les Maisons des sciences de l'Homme dans les sciences humaines et sociales, Paris, Rapport à Édouard Brezin pour le Comité d'initiative et de propositions des États généraux de la recherche et de l'enseignement supérieur.

GODELIER Maurice, (2001), L'état des sciences de la société en France et leur rôle dans la construction de l'espace européen de la recherche, Paris, Rapport au Premier ministre publié à La Documentation française.

IGAENR (Inspection générale de l'administration de l'Éducation nationale et de la Recherche), (2004), Les Maisons des sciences de l'Homme (MSH), Rapport par S. Srodogora, G. Lesage, J-R. Cytermann, M. Tyvaert et T. Chalon au ministre de l'Éducation nationale, de l'Enseignement supérieur et de la Recherche, et au ministre délégué à la Recherche, n²004-141, Paris : IGAENR. ftp : //trf. education. gouv. fr/pub/edutel/syst/igaen/rapports/msh_04. pdf

SUPIOT Alain (sous la direction de), (2001), Pour une politique des sciences de l'homme et de la société, Paris, PUF, coll. «Quadrige » 
Avenir de la recherche

Imprimé en France - SPRINT Impressions visionnaires Peripark, 101 Avenue Louis Roche, 92230 Gennevilliers

Dépôt légal : Janvier 2020 
Cette réédition, en 2020, de l'ouvrage paru en 2006 et consacré aux Maisons des sciences de l'homme permet de mettre en lumière les fondamentaux scientifiques et institutionnels à l'origine de la création des MSH et de leur réseau, déployé au cours des années 2000 sur le territoire national. Ces textes témoignent d'un projet initial particulièrement visionnaire.

Ce livre s'adresse à tous ceux qui s'intéressent aux sciences humaines et sociales et aux évolutions décisives pour les acteurs français. II est destiné à être complété par un deuxième volume qui couvrira la période 2012-2020.

C'est une initiative du Réseau national des MSH soutenue par l'alliance Athéna.

\section{Collection ATHENA \\ $\checkmark$ Alliance thématique nationale \\ ISBN : 979-10-93170-05-3}

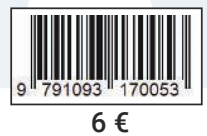

